\title{
A Unified Model for Stress-Driven Rearrangement Instabilities
}

\author{
Shokhrukh Yu. Kholmatovid \& Paolo Piovano
}

\author{
Communicated by I. FONSECA
}

\begin{abstract}
A variational model to simultaneously treat Stress-Driven Rearrangement Instabilities, such as boundary discontinuities, internal cracks, external filaments, edge delamination, wetting, and brittle fractures, is introduced. The model is characterized by an energy displaying both elastic and surface terms, and allows for a unified treatment of a wide range of settings, from epitaxially-strained thin films to crystalline cavities, and from capillarity problems to fracture models. The existence of minimizing configurations is established by adopting the direct method of the Calculus of Variations. The compactness of energy-equibounded sequences and energy lower semicontinuity are shown with respect to a proper selected topology in a class of admissible configurations that extends the classes previously considered in the literature. In particular, graph-like constraints previously considered for the setting of thin films and crystalline cavities are substituted by the more general assumption that the free crystalline interface is the boundary, consisting of an at most fixed finite number $m$ of connected components, of sets of finite perimeter. Finally, it is shown that, as $m \rightarrow \infty$, the energy of minimal admissible configurations tends to the minimum energy in the general class of configurations without the bound on the number of connected components for the free interface.
\end{abstract}

\section{Contents}

1. Introduction . . . . . . . . . . . . . . . . . . . . . . 416

2. Mathematical Setting . . . . . . . . . . . . . . . . . . . . 421

2.1. The Model . . . . . . . . . . . . . . . . . . . . . . . . . . . . . 422

2.2. Applications . . . . . . . . . . . . . . . . . . . . . 424

2.3. Main Results . . . . . . . . . . . . . . . . . . . . . . . . 425

3. Compactness . . . . . . . . . . . . . . . . . 428

4. Lower Semicontinuity . . . . . . . . . . . . . . . . . . . . . 450

5. Existence . . . . . . . . . . . . . . . . . . . . . . 475

Appendix A . . . . . . . . . . . . . . . . . . . . 481

A.1.Kuratowski Convergence . . . . . . . . . . . . . . . . . 481 
A.2. Rectifiability in $\mathbb{R}^{2} \ldots \ldots \ldots$. . . . . . . . . . . . . . 482

A.3. Minimizers of Volume-Constraint and Unconstraint Problems . . . . . . . . 484

References . . . . . . . . . . . . . . . . . . . 485

\section{Introduction}

Morphological destabilizations of crystalline interfaces are often referred to as Stress-Driven Rearrangement Instabilities (SDRI), from the seminal paper [40] (see also Asaro-Grinfeld-Tiller instabilities [4,21]). SDRI consist in various mechanisms of mass rearrangements that take place at crystalline boundaries because of the strong stresses originated by the mismatch between the parameters of adjacent crystalline lattices. Atoms move from their crystalline order and different modes of stress relief may co-occur, such as deformations of the bulk materials with storage of elastic energy, and boundary instabilities that contribute to the surface energy.

In this paper we introduce a variational model displaying both elastic and surface energy that simultaneously takes into account the various possible SDRI, such as boundary discontinuities, internal cracks, external filaments, wetting and edge delamination with respect to a substrate, and brittle fractures. In particular, the model provides a unified mathematical treatment of epitaxially-strained thin films $[22,31,33,42,48]$, crystal cavities [30,47,49], capillary droplets [11,24,26], as well as Griffith and failure models $[9,13,14,39,50]$, which were previously treated separately in the literature. Furthermore, the possibility of delamination and debonding, i.e., crack-like modes of interface failure at the interface with the substrate [27,41], is treated in accordance with the models in $[5,43,44]$, that were introduced by revisiting in the variational perspective of fracture mechanics the model first described in [50]. Notice that as a consequence the surface energy depends on the admissible deformations and cannot be decoupled from the elastic energy. As a byproduct of our analysis, we extend previous results for the existence of minimal configurations to anisotropic surface and elastic energies, and we relax constraints previously assumed on admissible configurations in the thin-film and crystal-cavity settings. For thin films we avoid the reduction considered in [22,23,31] to only film profiles parametrizable by thickness functions, and for crystal cavities the restriction in [30] to cavity sets consisting of only one connected starshaped void.

The class of interfaces that we consider is given by all the boundaries, that consists of connected components whose number is arbitrarily large but not exceeding a fixed number $m$, of sets of finite perimeter $A$. We refer to the class of sets of finite perimeter associated to the free interfaces as free crystals and we notice that free crystals $A$ may present an infinite number of components. The assumption on the number of components for the boundaries of free crystals is needed to apply an adaptation to our setting of the generalization of Golab's Theorem proven in [36] that allows one to establish in dimension 2, to which we restrict, compactness with respect to a proper selected topology. To the best of our knowledge, no variational framework able to guarantee the existence of minimizers in dimension 3 in the settings of thin films and crystal cavities is available in the literature.

Furthermore, the class of admissible deformations is enlarged with respect to $[22,23,30,31]$ to allow debonding and edge delamination to occur along the contact 


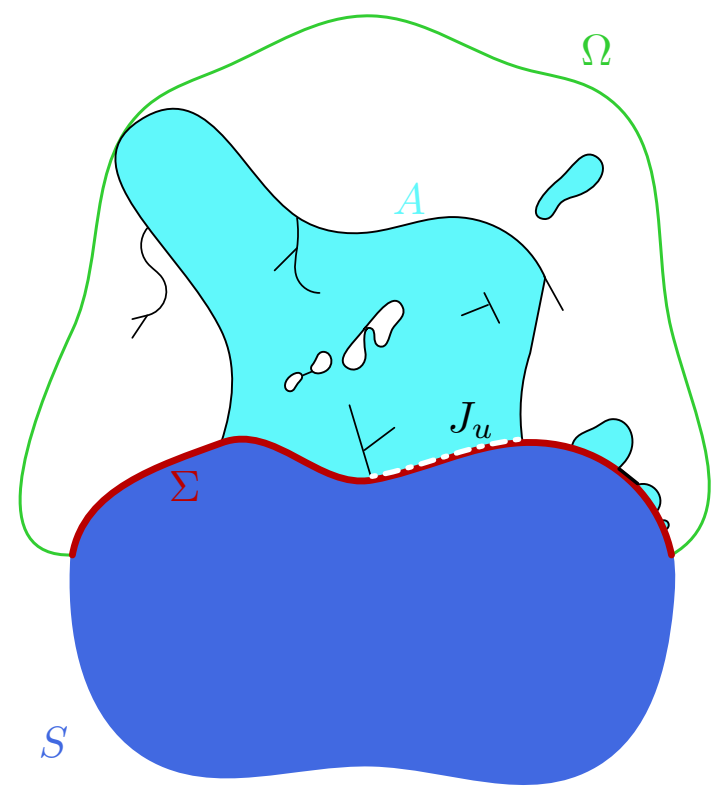

Fig. 1. An admissible free (disconnected) crystal $A$ is displayed in light blue in the container $\Omega$, while the substrate $S$ is represented in dark blue. The boundary of $A$ (with the cracks) is depicted in black, the container boundary in green, the contact surface $\Sigma$ in red (thicker line) while the delamination region $J_{u}$ with a white dashed line

surface $\Sigma:=\partial S \cap \partial \Omega$ between the fixed substrate $S$ and the fixed bounded region $\Omega$ containing the admissible free crystals (see Fig. 1 ). In what follows we refer to $\Omega$ as the container in analogy with capillarity problems. Notice that the obtained results can be easily applied also for unbounded containers in the setting of thin films with the graph constraint (see Section 2.2). Mathematically this is modeled by considering admissible deformations $u$ that are Sobolev functions only in the interior of the free crystals $A$ and the substrate $S$, and $G S B D$, i.e., generalized special functions of bounded deformation (see [19] for more details), on $A \cup S \cup \Sigma$. Thus, jumps $J_{u}$ that represent edge delamination can develop at the contact surface $\Sigma$, i.e., $J_{u} \subset \Sigma$.

The energy $\mathcal{F}$ that characterizes our model is defined for every admissible configuration $(A, u)$ in the configurational space $\mathcal{C}_{m}$ of free crystals and deformations by

$$
\mathcal{F}(A, u):=\mathcal{S}(A, u)+\mathcal{W}(A, u)
$$

where $\mathcal{S}$ denotes the surface energy and $\mathcal{W}$ the bulk elastic energy. The bulk elastic energy is given by

$$
\mathcal{W}(A, u)=\int_{A \cup S} W\left(z, e(u)-E_{0}\right) \mathrm{d} z
$$

for an elastic density $W(z, M):=\mathbb{C}(z) M: M$ defined with respect to a positivedefinite elasticity tensor $\mathbb{C}$ and a mismatch strain $E_{0}$. The mismatch strain is intro- 
duced to represent the fact that the lattice of the free crystal generally does not match the substrate lattice. We notice that the tensor $\mathbb{C}$ is assumed to be only $L^{\infty}(\Omega \cup S)$, therefore not only allowing for different elastic properties between the material of the free crystals in $\Omega$ and the one of the substrate, but also for non-constant properties in each material extending previous results. The surface energy $\mathcal{S}$ is defined as

$$
\mathcal{S}(A, u)=\int_{\partial A} \psi(z, u, v) \mathrm{d} \mathcal{H}^{d-1},
$$

with surface tension $\psi$ defined by

$$
\psi(z, u, v):= \begin{cases}\varphi\left(z, v_{A}(z)\right) & z \in \Omega \cap \partial^{*} A, \\ 2 \varphi\left(z, v_{A}(z)\right) & z \in \Omega \cap\left(A^{(1)} \cup A^{(0)}\right) \cap \partial A, \\ \varphi\left(z, v_{S}(z)\right)+\beta(z) & \Sigma \cap A^{(0)} \cap \partial A, \\ \beta(z) & z \in \Sigma \cap \partial^{*} A \backslash J_{u}, \\ \varphi\left(z, v_{S}(z)\right) & J_{u},\end{cases}
$$

where $\varphi \in C\left(\bar{\Omega} \times \mathbb{R}^{d} ;[0,+\infty)\right)$ is a Finsler norm representing the material anisotropy with $c_{1}|\xi| \leqq \varphi(x, \xi) \leqq c_{2}|\xi|$ for some $c_{1}, c_{2}>0, \beta \in L^{\infty}(\Sigma)$ is the relative adhesion coefficient on $\Sigma$ with

$$
|\beta(z)| \leqq \varphi\left(z, v_{S}(z)\right)
$$

for $z \in \Sigma, v$ is the exterior normal on the reduced boundary $\partial^{*} A$, and $A^{(\delta)}$ denotes the set of points of $A$ with density $\delta \in[0,1]$. Notice that the anisotropy $\varphi$ is counted double on the sets $A^{(1)} \cap \partial A \cap \Omega$ and $A^{(0)} \cap \partial A \cap \Omega$, that represent the set of cracks and the set of external filaments, respectively. On the free profile $\partial^{*} A$ the anisotropy is weighted the same as on the delamination region $J_{u}$, since delamination involves debonding between the adjacent materials by definition. Furthermore, the adhesion coefficient $\beta$ is considered on the contact surface $\Sigma$, alone on the reduced boundary $\Sigma \cap \partial^{*} A \backslash J_{u}$ and together with $\varphi$ on those external filaments $A^{(0)} \cap \partial A \cap \Sigma$, to which we refer as wetting layer.

We refer the Reader to Section 2.3 for the rigorous mathematical setting and the main results of the paper, among which we recall here the following existence result:

Main Theorem If $\mathrm{V} \in(0,|\Omega|)$ or $S=\emptyset$, then for every $m \geqq 1$ the volumeconstrained minimum problem

$$
\inf _{(A, u) \in \mathcal{C}_{m},|A|=\mathrm{v}} \mathcal{F}(A, u)
$$

admits a solution and

$$
\inf _{(A, u) \in \mathcal{C},|A|=\mathrm{v}} \mathcal{F}(A, u)=\lim _{m \rightarrow \infty} \inf _{(A, u) \in \mathcal{C}_{m},|A|=\mathrm{v}} \mathcal{F}(A, u) .
$$

This existence result is accomplished in Theorem 2.6, where we also solve the related unconstraint problem with energy $\mathcal{F}^{\lambda}$ given by $\mathcal{F}$ plus a volume penalization depending on the parameter $\lambda>0$. 
The proof is based on the direct method of the Calculus of Variations, i.e., it consists in determining a suitable topology $\tau_{\mathcal{C}}$ in $\mathcal{C}_{m}$ sufficiently weak to establish the compactness of energy-equibounded sequences in Theorem 2.7 and strong enough to prove that the energy is lower semicontinuous in Theorem 2.8. We notice here that Theorems 2.7 and 2.8 can also be seen as an extension, under the condition on the maximum admissible number $m$ of connected components for the boundary, of the compactness and lower semicontinuity results in [15] to anisotropic surface tensions and to the other SDRI settings.

The topology $\tau_{\mathcal{C}}$ selected in $\mathcal{C}$ corresponds, under the uniform bound on the length of the free-crystal boundaries, to the convergence of both the free crystals and the free-crystal complementary sets with respect to the Kuratowski convergence and to the pointwise convergence of the displacements. In $[22,23,31]$ the weaker convergence $\tau_{\mathcal{C}}^{\prime}$ consisting of only the Kuratowski convergence of complementary sets of free-crystals (together with the $\bar{S}$ ) was considered, which in our setting without graph-like assumptions on the free boundary is not enough because not closed in $\mathcal{C}_{m}$. Working with the topology $\tau_{\mathcal{C}}$ also allows as to keep track or the surface energy of the possible external filaments of the admissible free crystals, which were in previous results not considered. However, to establish compactness with respect to $\tau_{\mathcal{C}}$ the Blaschke Selection Theorem employed in [22,23,30,31] is not enough, and a version for the signed distance functions from the free boundaries is obtained (see Proposition 3.1). Furthermore, in order to take in consideration the situation in which connected components of $A_{k}$ separates in the limit in multiple connected components of $A$, e.g., in the case of neckpinches, we need to introduce extra boundary in $A_{k}$ in order to divide their components accordingly (see Proposition 3.6). Otherwise, adding to $u_{k}$ different rigid displacements with respect to the components in $A$ (which are needed for compactness of $u_{k}$ ) would results in jumps for the displacements in $A_{k}$, which are not allowed in our setting with $H_{\text {loc }}^{1}$ displacements. Therefore, we pass from the sequence $A_{k}$ to a sequence $D_{k}$ with such extra boundary for which we can prove compactness. Passing to $D_{k}$ is not a problem in the existence in view of property (2.9) that relates the $\liminf$ of the energy with respect to $A_{k}$ to the one with respect to $D_{k}$. However, in case $S \neq \emptyset$, in order to prove (2.9), we need to further modify the sequence $D_{k}$ from the original $A_{k}$ by cutting out the portion converging to delamination regions (e.g., portion containing accumulating cracks and voids at the boundary with $S$ ) using Proposition 3.9, and, in order to maintain the volume constraint, by replacing them with an extra set that does not contribute to the overall elastic energy.

The lower semicontinuity of the energy with respect to $\tau_{\mathcal{C}}$ is established for the elastic energy as in [31] by convexity, and for the surface energy in Proposition 4.1 in several steps by adopting a blow-up method (see, e.g., [1,8]). More precisely, given a sequence of configurations $\left(A_{k}, u_{k}\right) \in \mathcal{C}_{m}$ converging to $(A, u) \in \mathcal{C}_{m}$ we consider a converging subsequence of the Radon measures $\mu_{k}$ associated to the surface energy and $\left(A_{k}, u_{k}\right)$, and we estimate from below the Radon-Nikodym derivative of their limit denoted by $\mu_{0}$ with respect to the Hausdorff measure restricted to the 5 portions of $\partial A$ that appear in the definition of the surface anisotropy $\psi$ in (1.1). We overcome the fact that in general $\mu_{0}$ is not a non-negative measure due to the presence of the contact term in the energy with $\beta$, by adding to $\mu_{k}$ and $\mu_{0}$ 
the positive measure

$$
\mu_{\Sigma}(B)=\int_{B \cap \Sigma} \varphi\left(x, v_{\Sigma}(x)\right) \mathrm{d} \mathcal{H}^{1}
$$

defined for every Borel set $B \subset \mathbb{R}^{2}$ and using (1.2). The estimates for the RadonNikodym derivative related to the free boundary $\Omega \cap \partial^{*} A$ and the contact region $\left(\Sigma \cap \partial^{*} A\right) \backslash J_{u_{k}}$ follow from [1, Lemma 3.8]. For the estimates related to exterior filaments and interior cracks we first separately reduce to the case of flat filaments and cracks, and then we adapt some arguments from [36]. Extra care is needed to treat the exterior filament lying on $\Sigma$ to which we refer as wetting layer in analogy to the thin-film setting. The estimate related to the delamination region on $\Sigma$ follows by blow-up under condition (4.2) that ensures that the delamination regions between the limiting free crystal $A$ and the substrate $S$ can be originated from delamination regions between $A_{k}$ and $S$ and from portions of free boundaries $\partial^{*} A_{k}$ or interior cracks collapsing on $\Sigma$, as well as from accumulation of interior cracks starting from $\left(\Sigma \cap \partial^{*} A_{k}\right) \backslash J_{u_{k}}$.

A challenging point is to prove that condition (4.2) is satisfied by $\left(A_{k}, u_{k}\right)$. In order to do this, in Theorem 2.8 we first extend the displacements $u_{k}$ to the set $\Omega \backslash\left(A_{k} \cup S\right)$ using Lemma 4.8. The extension of the $u_{k}$ is performed without creating extra jump at the interface on the exposed surface of the substrate, i.e., the jump set of the extensions is approximately $J_{u_{k}} \cup\left(\Omega \cap \partial A_{k}\right)$. We point out that as a consequence we obtain also in Proposition 4.9 the lower semicontinuity, with respect to the topology $\tau_{\mathcal{C}}^{\prime}$, of a version of our energy without exterior filaments (but with wetting layer) extending the lower semicontinuity results of [22,30,31].

Finally, we prove (1.3), which entails the existence of a minimizing sequence $\left(A_{m}, u_{m}\right) \in \mathcal{C}_{m}$ for the minimum problem of $\mathcal{F}$ in $\mathcal{C}$. This is obtained by considering a minimizing sequence $\left(A_{\varepsilon}, u_{\varepsilon}\right) \in \mathcal{C}$ for $\mathcal{F}^{\lambda}$, and then by modifying it into a new minimizing sequence $\left(E_{\varepsilon, \lambda}, v_{\varepsilon, \lambda}\right) \in \mathcal{C}_{m}$ such that $\mathcal{F}^{\lambda}\left(A_{\varepsilon}, u_{\varepsilon}\right)+\delta_{\varepsilon} \geqq$ $\mathcal{F}^{\lambda}\left(E_{\varepsilon, \lambda}, v_{\varepsilon, \lambda}\right)$ for some $\delta_{\varepsilon} \rightarrow 0$ as $\varepsilon \rightarrow 0$. The construction of $\left(E_{\varepsilon, \lambda}, v_{\varepsilon, \lambda}\right) \in \mathcal{C}_{m}$ requires 2 steps. In the first step we eliminate the external filaments, we remove sufficiently small connected components of $A_{\varepsilon}$, and we fill in sufficiently small holes till we reach a finite number of connected components with a finite number of holes (see Fig. 2). In the second step we redefine the deformations in the free crystal by employing [14, Theorem 1.1] in order to obtain a deformation with jump set consisting of at most finitely many components, and such that the difference in the elastic energy and the length of the jump sets with respect to $u_{\varepsilon}$ remains small.

The paper is organized as follows: in Section 2 we introduce the model and the topology $\tau_{\mathcal{C}}$, we refer to various SDRI settings from the literature that are included in our analysis, and we state the main results. In Section 3 we prove sequential compactness for the free crystals with the bound $m$ on the boundary components in Proposition 3.3 and for $\mathcal{C}_{m}$ in Theorem 2.7. In Section 4 we prove the lower semicontinuity of the energy (Theorem 2.8 ) by first considering only the surface energy $\mathcal{S}$ under the condition (4.2) (see Proposition 4.1), and we conclude the section by showing the lower semicontinuity of the energy without the external filament and wetting-layer terms with respect to the topology $\tau_{\mathcal{C}}^{\prime}$ (see Proposition 4.9). In Section 5 we prove the existence results (Theorems 2.6 and 2.9) and property 
(1.3). The paper is concluded with an Appendix where results related to rectifiable sets and Kuratowski convergence are recalled for reader's convenience.

\section{Mathematical Setting}

We start by introducing some notation. Since our model is two-dimensional, unless otherwise stated, all sets we consider are subsets of $\mathbb{R}^{2}$. We choose the standard basis $\left\{\mathbf{e}_{1}=(1,0), \mathbf{e}_{2}=(0,1)\right\}$ in $\mathbb{R}^{2}$ and denote the coordinates of $x \in \mathbb{R}^{2}$ with respect to this basis by $\left(x_{1}, x_{2}\right)$. We denote by $\operatorname{Int}(A)$ the interior of $A \subset \mathbb{R}^{2}$. Given a Lebesgue measurable set $E$, we denote by $\chi_{E}$ its characteristic function and by $|E|$ its Lebesgue measure. The set

$$
E^{(\alpha)}:=\left\{x \in \mathbb{R}^{2}: \lim _{r \rightarrow 0} \frac{\left|E \cap B_{r}(x)\right|}{\left|B_{r}(x)\right|}=\alpha\right\}, \quad \alpha \in[0,1],
$$

where $B_{r}(x)$ denotes the ball in $\mathbb{R}^{2}$ centered at $x$ of radius $r>0$, is called the set of points of density $\alpha$ of $E$. Clearly, $E^{(\alpha)} \subset \partial E$ for any $\alpha \in(0,1)$, where

$$
\partial E:=\left\{x \in \mathbb{R}^{2}: B_{r}(x) \cap E \neq \emptyset \text { and } B_{r}(x) \backslash E \neq \emptyset \text { for any } r>0\right\}
$$

is the topological boundary. The set $E^{(1)}$ is the Lebesgue set of $E$ and $\left|E^{(1)} \Delta E\right|=0$. We denote by $\partial^{*} E$ the reduced boundary of a finite perimeter set $E[3,37]$, i.e.,

$$
\partial^{*} E:=\left\{x \in \mathbb{R}^{2}: \exists v_{E}(x):=-\lim _{r \rightarrow 0} \frac{D \chi_{E}\left(B_{r}(x)\right)}{\left|D \chi_{E}\right|\left(B_{r}(x)\right)}, \quad\left|v_{E}(x)\right|=1\right\} .
$$

The vector $v_{E}(x)$ is called the measure-theoretic normal to $\partial E$.

The symbol $\mathcal{H}^{s}, s \geqq 0$, stands for the $s$-dimensional Hausdorff measure. An $\mathcal{H}^{1}$-measurable set $K$ with $0<\mathcal{H}^{1}(K)<\infty$ is called $\mathcal{H}^{1}$-rectifiable if $\theta^{*}(K, x)=$ $\theta_{*}(K, x)=1$ for $\mathcal{H}^{1}$-a.e. $x \in K$, where

$$
\theta^{*}(K, x):=\limsup _{r \rightarrow 0^{+}} \frac{\mathcal{H}^{1}\left(B_{r}(x) \cap K\right)}{2 r}, \quad \theta_{*}(K, x):=\liminf _{r \rightarrow 0^{+}} \frac{\mathcal{H}^{1}\left(B_{r}(x) \cap K\right)}{2 r} .
$$

By [29, Theorem 2.3] any $\mathcal{H}^{1}$-measurable set $K$ with $0<\mathcal{H}^{1}(K)<\infty$ satisfies $\theta^{*}(K, x)=1$ for $\mathcal{H}^{1}$-a.e. $x \in K$.

Remark 2.1. If $E$ is a finite perimeter set, then

(a) $\overline{\partial^{*} E}=\partial E^{(1)}$ (see, e.g., [37, Theorem 4.4] and [46, Eq. 15.3]);

(b) $\partial^{*} E \subseteq E^{(1 / 2)}$ and $\mathcal{H}^{1}\left(E^{(1 / 2)} \backslash \partial^{*} E\right)=0$ (see, e.g., [3, Theorem 3.61] and [46, Theorem 16.2]);

(c) $P(E, B)=\mathcal{H}^{1}\left(B \cap \partial^{*} E\right)=\mathcal{H}^{1}\left(B \cap E^{(1 / 2)}\right)$ for any Borel set $B$.

The notation $\operatorname{dist}(\cdot, E)$ stands for the distance function from the set $E \subset \mathbb{R}^{2}$ with the convention that $\operatorname{dist}(\cdot, \varnothing) \equiv+\infty$. Given a set $A \subset \mathbb{R}^{2}$, we consider also signed distance function from $\partial A$, negative inside, defined as

$$
\operatorname{sdist}(x, \partial A):= \begin{cases}\operatorname{dist}(x, A) & \text { if } x \in \mathbb{R}^{2} \backslash A, \\ -\operatorname{dist}\left(x, \mathbb{R}^{2} \backslash A\right) & \text { if } x \in A\end{cases}
$$


Remark 2.2. The following assertions are equivalent:

(a) $\operatorname{sdist}\left(x, \partial E_{k}\right) \rightarrow \operatorname{sdist}(x, \partial E)$ locally uniformly in $\mathbb{R}^{2}$;

(b) $E_{k} \stackrel{\mathcal{K}}{\rightarrow} \bar{E}$ and $\mathbb{R}^{2} \backslash E_{k} \stackrel{\mathcal{K}}{\rightarrow} \mathbb{R}^{2} \backslash \operatorname{Int}(E)$, where $\mathcal{K}$-Kuratowski convergence of sets [18, Chapter 4].

Moreover, either assumption implies $\partial E_{k} \stackrel{\mathcal{K}}{\rightarrow} \partial E$.

\subsection{The Model}

Given two open sets $\Omega \subset \mathbb{R}^{2}$ and $S \subset \mathbb{R}^{2} \backslash \Omega$, we define the family of admissible regions for the free crystal and the space of admissible configurations by

$$
\mathcal{A}:=\left\{A \subset \bar{\Omega}: \partial A \text { is } \mathcal{H}^{1}-\text { rectifiable and } \mathcal{H}^{1}(\partial A)<\infty\right\}
$$

and

$$
\begin{aligned}
\mathcal{C}:=\{(A, u): A & \in \mathcal{A}, \\
& \left.u \in G S B D^{2}\left(\operatorname{Int}(A \cup S \cup \Sigma) ; \mathbb{R}^{2}\right) \cap H_{\mathrm{loc}}^{1}\left(\operatorname{Int}(A) \cup S ; \mathbb{R}^{2}\right)\right\},
\end{aligned}
$$

respectively, where $\Sigma:=\partial S \cap \partial \Omega$ and $G S B D^{2}\left(E, \mathbb{R}^{2}\right)$ is the collection of all generalized special functions of bounded deformation $[15,19]$. Given a displacement field $u \in G S B D^{2}\left(\operatorname{Int}(A \cup S \cup \Sigma) ; \mathbb{R}^{2}\right) \cap H_{\text {loc }}^{1}\left(\operatorname{Int}(A) \cup S ; \mathbb{R}^{2}\right)$ we denote by $e(u(\cdot))$ the density of $\mathbf{e}(u)=\left(D u+(D u)^{T}\right) / 2$ with respect to Lebesgue measure $\mathcal{L}^{2}$ and by $J_{u}$ the jump set of $u$. Recall that $e(u) \in L^{2}(A \cup S)$ and $J_{u}$ is $\mathcal{H}^{1}$-rectifiable. Notice also that assumption $u \in H_{\mathrm{loc}}^{1}\left(\operatorname{Int}(A) \cup S ; \mathbb{R}^{2}\right)$ implies $J_{u} \subset \Sigma \cap \overline{\partial^{*} A}$. We denote the boundary trace of a function $u: A \rightarrow \mathbb{R}^{n}$ by $\operatorname{tr}_{A}$ (if exists).

Remark 2.3. For any $A \in \mathcal{A}$ :

(a) $\partial A=N \cup\left(\Omega \cap \partial^{*} A\right) \cup(\partial \Omega \cap \partial A) \cup\left(\Omega \cap A^{(0)} \cap \partial A\right) \cup\left(\Omega \cap A^{(1)} \cap \partial A\right)$, where $N$ is an $\mathcal{H}^{1}$-negligible set (see, e.g., [46, page 184]);

(b) $\Omega \cap \overline{\partial^{*} A}=\Omega \cap \partial A^{(1)}$ (see Remark 2.1 (a) above);

(c) $\mathcal{H}^{1}\left(\overline{\partial^{*} A} \backslash \partial^{*} A\right)=0$ (since $\overline{\partial^{*} A} \subset \partial A$ is $\mathcal{H}^{1}$-rectifiable);

(d) up to a $\mathcal{H}^{1}$-negligible set, the trace of $A \in \mathcal{A}$ on $\partial \Omega$ is defined as $\partial \Omega \cap \partial^{*} A$ (see, e.g., [1, Lemma 2.10]).

Unless otherwise stated, in what follows $\Omega$ and $S \subset \mathbb{R}^{2} \backslash \Omega$ are bounded Lipschitz open sets with finitely many connected components satisfying $\mathcal{H}^{1}(\partial S)+$ $\mathcal{H}^{1}(\partial \Omega)<\infty$ and $\Sigma \subseteq \partial \Omega$ is a Lipschitz 1-manifold.

We introduce in $\mathcal{A}$ the following notion of convergence:

Definition 2.4. ( $\tau_{\mathcal{A}}$-Convergence) A sequence $\left\{A_{k}\right\} \subset \mathcal{A}$ is said to $\tau_{\mathcal{A}}$-converge to $A \subset \mathbb{R}^{2}$ and is written $A_{k} \stackrel{\tau_{\mathcal{A}}}{\rightarrow} A$ if

$$
\begin{aligned}
& -\sup _{k \geqq 1} \mathcal{H}^{1}\left(\partial A_{k}\right)<\infty ; \\
& -\operatorname{sdist}\left(\cdot, \partial A_{k}\right) \rightarrow \operatorname{sdist}(\cdot, \partial A) \text { locally uniformly in } \mathbb{R}^{2} \text { as } k \rightarrow \infty .
\end{aligned}
$$


We endow $\mathcal{C}$ with the following notion of convergence:

Definition 2.5. ( $\tau_{\mathcal{C}}$-Convergence) A sequence $\left\{\left(A_{n}, u_{n}\right)\right\} \subset \mathcal{C}$ is said to $\tau_{\mathcal{C}^{-}}$ converge to $(A, u) \in \mathcal{C}$, and is written $\left(A_{n}, u_{n}\right) \stackrel{\tau_{\mathcal{C}}}{\rightarrow}(A, u)$ if

$$
\begin{aligned}
& -A_{n} \stackrel{\tau_{\mathcal{A}}}{\rightarrow} A, \\
& -u_{n} \rightarrow u \text { a.e. } \operatorname{in} \operatorname{Int}(A) \cup S .
\end{aligned}
$$

The energy of admissible configurations is given by the functional $\mathcal{F}: \mathcal{C} \rightarrow$ $[-\infty,+\infty]$

$$
\mathcal{F}:=\mathcal{S}+\mathcal{W}
$$

where $\mathcal{S}$ and $\mathcal{W}$ are the surface and elastic energies of the configuration, respectively. The surface energy of $(A, u) \in \mathcal{C}$ is defined as

$$
\begin{aligned}
\mathcal{S}(A, u):= & \int_{\Omega \cap \partial^{*} A} \varphi\left(x, v_{A}(x)\right) \mathrm{d} \mathcal{H}^{1}(x) \\
& +\int_{\Omega \cap\left(A^{(1)} \cup A^{(0)}\right) \cap \partial A}\left(\varphi\left(x, v_{A}(x)\right)+\varphi\left(x,-v_{A}(x)\right)\right) \mathrm{d} \mathcal{H}^{1}(x) \\
& +\int_{\Sigma \cap A^{(0)} \cap \partial A}\left(\varphi\left(x, v_{\Sigma}(x)\right)+\beta(x)\right) \mathrm{d} \mathcal{H}^{1}(x) \\
& +\int_{\Sigma \cap \partial^{*} A \backslash J_{u}} \beta(x) \mathrm{d} \mathcal{H}^{1}(x)+\int_{J_{u}} \varphi\left(x,-v_{\Sigma}(x)\right) \mathrm{d} \mathcal{H}^{1}(x),
\end{aligned}
$$

where $\varphi: \bar{\Omega} \times \mathbb{S}^{1} \rightarrow[0,+\infty)$ and $\beta: \Sigma \rightarrow \mathbb{R}$ are Borel functions denoting the anisotropy of crystal and the relative adhesion coefficient of the substrate, respectively, and $v_{\Sigma}:=v_{S}$. In the following we refer to the first term in (2.2) as the free-boundary energy, to the second as the energy of internal cracks and external filaments, to the third as the wetting-layer energy, to the fourth as the contact energy, and to the last as the delamination energy. In applications instead of $\varphi(x, \cdot)$ it is more convenient to use its positively one-homogeneous extension $|\xi| \varphi(x, \xi /|\xi|)$. With a slight abuse of notation we denote this extension also by $\varphi$.

The elastic energy of $(A, u) \in \mathcal{C}$ is defined as

$$
\mathcal{W}(A, u):=\int_{A \cup S} W\left(x, e(u(x))-E_{0}(x)\right) \mathrm{d} x,
$$

where the elastic density $W$ is determined as the quadratic form

$$
W(x, M):=\mathbb{C}(x) M: M,
$$

by the so-called stress-tensor, a measurable function $x \in \Omega \cup S \rightarrow \mathbb{C}(x)$, where $\mathbb{C}(x)$ is a non-negative fourth-order tensor in the Hilbert space $\mathbb{M}_{\text {sym }}^{2 \times 2}$ of all $2 \times 2$ symmetric matrices with the natural inner product

$$
M: N=\sum_{i, j=1}^{2} M_{i j} N_{i j}
$$

\footnotetext{
${ }^{1}$ If $\mathbb{R}^{2} \backslash A_{k} \stackrel{K}{\rightarrow} \mathbb{R} \backslash \operatorname{Int}(A)$, then for any $x \in \operatorname{Int}(A)$ one has $x \in \operatorname{Int}\left(A_{n}\right)$ for all large $n$.
} 
for $M=\left(M_{i j}\right)_{1 \leqq i, j \leqq 2}, N=\left(N_{i j}\right)_{1 \leqq i, j \leqq 2} \in \mathbb{M}_{\text {sym }}^{2 \times 2}$.

The mismatch strain $x \in \Omega \cup S \mapsto E_{0}(x) \in \mathbb{M}_{\text {sym }}^{2 \times 2}$ is given by

$$
E_{0}:= \begin{cases}e\left(u_{0}\right) & \text { in } \Omega \\ 0 & \text { in } S\end{cases}
$$

for a fixed $u_{0} \in H^{1}(\Omega)$.

Given $m \geqq 1$, let $\mathcal{A}_{m}$ be a collection of all subsets $A$ of $\bar{\Omega}$ such that $\partial A$ has at most $m$ connected components. Recall that since $\partial A$ is closed, it is $\mathcal{H}^{1}$-measurable. By Proposition A.2, $\partial A$ is $\mathcal{H}^{1}$-rectifiable so that $\mathcal{A}_{m} \subset \mathcal{A}$. We call the set

$$
\mathcal{C}_{m}:=\left\{(A, u) \in \mathcal{C}: A \in \mathcal{A}_{m}\right\}
$$

the set of constrained admissible configurations. We also consider a volume constraint with respect to $v \in(0,|\Omega|]$, i.e.,

$$
|A|=\mathrm{V}
$$

for every $A \in \mathcal{A}$.

\subsection{Applications}

The model introduced in this paper includes the settings of various free boundary problems, some of which are outlined below.

- Epitaxially-strained thin films $[10,22,23,31,35]: \Omega:=(a, b) \times(0,+\infty), S:=$ $(a, b) \times(-\infty, 0)$ for some $a<b$, free crystals in the subfamily

$$
\mathcal{A}_{\text {subgraph }}:=\left\{A \subset \Omega: \exists h \in B V(\Sigma ;[0, \infty)) \text { and l.s.c. such that } A=A_{h}\right\} \subset \mathcal{A}_{1},
$$

where $A_{h}:=\left\{\left(x^{1}, x^{2}\right): 0<x^{2}<h\left(x^{1}\right)\right\}$, and admissible configurations in the subspace

$$
\mathcal{C}_{\text {subgraph }}:=\left\{(A, u): A \in \mathcal{A}_{\text {subgraph }}, u \in H_{\text {loc }}^{1}\left(\operatorname{Int}(A \cup S \cup \Sigma) ; \mathbb{R}^{2}\right)\right\} \subset \mathcal{C}_{1}
$$

(see also $[6,38]$ ). Notice that the container $\Omega$ is not bounded, however, we can reduce to the situation of bounded containers where we can apply Theorem 2.9 since every energy equibounded sequence in $\mathcal{A}_{\text {subgraph }}$ is contained in an auxiliary bounded set (see also Remark 2.10).

- Crystal cavities $[30,34,47,49]: \Omega \subset \mathbb{R}^{2}$ smooth set containing the origin, $S:=$ $\mathbb{R}^{2} \backslash \Omega$, free crystals in the subfamily

$$
\mathcal{A}_{\text {starshaped }}:=\{A \subset \Omega \text { : open, starshaped with respect to }(0,0) \text {, and } \partial \Omega \subset \partial A\} \subset \mathcal{A}_{1},
$$

and the space of admissible configurations

$\mathcal{C}_{\text {starshaped }}:=\left\{(A, u): A \in \mathcal{A}_{\text {starshaped }}, u \in H_{\text {loc }}^{1}\left(\operatorname{Int}(A \cup S \cup \Sigma) ; \mathbb{R}^{2}\right)\right\} \subset \mathcal{C}_{1}$.

See Remark 2.10. 
- Capillarity droplets, e.g., [11,24,26]: $\Omega \subset \mathbb{R}^{2}$ is a bounded open set (or a cylinder), $\mathbb{C}=0, S=\emptyset$, and admissible configurations in the collection

$$
\mathcal{C}_{\text {capillary }}:=\{(A, 0): A \in \mathcal{A}\} \subset \mathcal{C} .
$$

- Griffith fracture model, e.g., $[12,13,16,17]: S=\Sigma=\emptyset E_{0} \equiv 0$, and the space of configurations

$\mathcal{C}_{\text {Griffith }}:=\left\{(\Omega \backslash K, u): K\right.$ closed, $\mathcal{H}^{1}-$ rectifiable, $\left.u \in H_{\text {loc }}^{1}\left(\Omega \backslash K ; \mathbb{R}^{2}\right)\right\} \subset \mathcal{C}$.

- Mumford-Shah model (without fidelity term), e.g., [3,20,45]: $S=\Sigma=\varnothing$, $E_{0}=0, \mathbb{C}$ is such that the elastic energy $\mathcal{W}$ reduces to the Dirichlet energy, and the space of configurations

$$
\mathcal{C}_{\text {Mumfard-Shah }}:=\left\{(\Omega \backslash K, u) \in \mathcal{C}_{\text {Griffith }}: u=\left(u_{1}, 0\right)\right\} \subset \mathcal{C} .
$$

- Boundary delaminations [5,27,41,43,44,50]: the setting of our model finds applications to describe debonding and edge delaminations in composites [50]. We notice that our perspective differs from $[5,43,44]$ where reduced models for the horizontal interface between the film and the substrate are derived, since instead we focus on the 2-dimensional film and substrate vertical section.

\subsection{Main Results}

In this subsection we state the main results of the paper. Let us formulate our main hypotheses:

(H1) $\varphi \in C\left(\bar{\Omega} \times \mathbb{R}^{2} ;[0,+\infty)\right)$ and is a Finsler norm, i.e., there exist $c_{2} \geqq c_{1}>0$ such that for every $x \in \bar{\Omega}, \varphi(x, \cdot)$ is a norm in $\mathbb{R}^{2}$ satisfying

$$
c_{1}|\xi| \leqq \varphi(x, \xi) \leqq c_{2}|\xi| \quad \text { for any } x \in \bar{\Omega} \text { and } \xi \in \mathbb{R}^{2}
$$

(H2) $\beta \in L^{\infty}(\Sigma)$ and satisfies

$$
-\varphi\left(x, v_{\Sigma}(x)\right) \leqq \beta(x) \leqq \varphi\left(x, v_{\Sigma}(x)\right) \quad \text { for } \mathcal{H}^{1}-\text { a.e. } x \in \Sigma
$$

(H3) $W$ is of the form (2.3) with $\mathbb{C} \in L^{\infty}(\Omega \cup S)$ such that

$$
\mathbb{C}(x) M: M \geqq 2 c_{3} M: M \quad \text { for any } M \in \mathbb{M}_{\text {sym }}^{2 \times 2}
$$

for some $c_{3}>0$.

Theorem 2.6. (Existence) Assume (H1)-(H3). Let either $\mathrm{v} \in(0,|\Omega|)$ or $S=\emptyset$. Then for every $m \geqq 1, \lambda>0$ both the volume-constrained minimum problem

$$
\inf _{(A, u) \in \mathcal{C}_{m},|A|=\mathrm{v}} \mathcal{F}(A, u),
$$

and the unconstrained minimum problem

$$
\inf _{(A, u) \in \mathcal{C}_{m}} \mathcal{F}^{\lambda}(A, u)
$$


have a solution, where $\mathcal{F}^{\lambda}: \mathcal{C}_{m} \rightarrow \mathbb{R}$ is defined as

$$
\mathcal{F}^{\lambda}(A, u):=\mathcal{F}(A, u)+\lambda|| A|-\mathrm{v}| .
$$

Furthermore, there exists $\lambda_{0}>0$ such that for every $\mathrm{v} \in(0,|\Omega|]$ and $\lambda>\lambda_{0}$,

$$
\inf _{(A, u) \in \mathcal{C},|A|=\mathrm{v}} \mathcal{F}(A, u)=\inf _{(A, u) \in \mathcal{C}} \mathcal{F}^{\lambda}(A, u)=\lim _{m \rightarrow \infty} \inf _{(A, u) \in \mathcal{C}_{m},|A|=\mathrm{v}} \mathcal{F}(A, u) .
$$

We notice that for $\lambda>\lambda_{0}$ solutions of (CP) and (UP) coincide (see the proof of Theorem 2.6) for any $\mathrm{V} \in(0,|\Omega|]$ and $m \geqq 1$. Moreover, (2.7) shows that a minimizing sequence for $\mathcal{F}$ in $\mathcal{C}$ can be chosen among the sets whose boundary have finitely many connected components.

The proof of the existence part of Theorem 2.6 is given mainly by the following two results in which we show that $\mathcal{C}_{m}$ is $\tau_{\mathcal{C}}$-compact and $\mathcal{F}$ is $\tau_{\mathcal{C}}$-lower semicontinuous. Recall that an (infinitesimal) rigid displacement in $\mathbb{R}^{n}$ is an affine transformation $a(x)=M x+b$, where $M$ is a skew-symmetric (i.e., $M^{T}=-M$ ) $n \times n$-matrix and $b \in \mathbb{R}^{n}$. Given $B \in \mathcal{A}$ with $\operatorname{Int}(B)=\cup_{j} E_{j}$, where $\left\{E_{j}\right\}$ are all connected components of $\operatorname{Int}(B)$, we say the function

$$
a=\sum_{j \geqq 1}\left(M_{j} x+b_{j}\right) \chi_{E_{j}},
$$

a piecewise rigid displacement associated to $B$, here $M_{j} x+b_{j}$ is a rigid displacement in $\mathbb{R}^{2}$.

Theorem 2.7. (Compactness of $\left.\mathcal{C}_{m}\right)$ Assume $(\mathrm{H} 1)-(\mathrm{H} 3)$. Let either $\mathrm{v} \in(0,|\Omega|)$ or $S=\emptyset$. Let $\left\{\left(A_{k}, u_{k}\right)\right\} \subset \mathcal{C}_{m}$ be such that

$$
\sup _{k \geqq 1} \mathcal{F}\left(A_{k}, u_{k}\right)<\infty
$$

and

$$
\left|A_{k}\right| \leqq \mathrm{V}
$$

for every $k \geqq 1$. Then there exist $(A, u) \in \mathcal{C}_{m}$ of finite energy, a subsequence $\left\{\left(A_{k_{n}}, u_{k_{n}}\right)\right\}$ and a sequence $\left\{\left(D_{n}, v_{n}\right)\right\} \subset \mathcal{C}_{m}$ with

$$
v_{n}:=\left(u_{k_{n}}+a_{n}\right) \chi_{D_{n} \cap A_{k_{n}}}+u_{0} \chi_{D_{n} \backslash A_{k_{n}}}
$$

for some piecewise rigid displacements $a_{n}$ associated to $D_{n}$, such that $A_{k_{n}} \stackrel{{ }^{\tau_{\mathcal{A}}}}{\rightarrow} A$, $\left(D_{n}, v_{n}\right) \stackrel{\tau_{\mathcal{C}}}{\rightarrow}(A, u),\left|D_{n}\right|=\left|A_{k_{n}}\right|$, and

$$
\liminf _{n \rightarrow \infty} \mathcal{F}\left(A_{k_{n}}, u_{k_{n}}\right) \geqq \liminf _{n \rightarrow \infty} \mathcal{F}\left(D_{n}, v_{n}\right) .
$$

Theorem 2.8. (Lower semicontinuity of $\mathcal{F})$ Assume $(H 1)-(H 3)$ and let $\left\{\left(A_{k}, u_{k}\right)\right\} \subset$ $\mathcal{C}_{m}$ and $(A, u) \in \mathcal{C}_{m}$ be such that $\left(A_{k}, u_{k}\right) \stackrel{{ }^{\mathcal{C}}}{\rightarrow}(A, u)$. Then

$$
\liminf _{k \rightarrow \infty} \mathcal{F}\left(A_{k}, u_{k}\right) \geqq \mathcal{F}(A, u) .
$$


As a byproduct of our methods we obtain the following existence result in a subspace of $\mathcal{C}_{m}$ with respect to a weaker topology previously used in $[22,30,31]$ for thin films and crystal cavities.

Theorem 2.9. (Existence for weaker topology) Assume (H1)-(H3) and fix $m \geqq 1$ and $\mathrm{v} \in(0,|\Omega|]$. The functional $\mathcal{F}^{\prime}: \mathcal{C} \rightarrow \mathbb{R}$ defined as

$$
\begin{aligned}
\mathcal{F}^{\prime}(A, u):=\mathcal{F}(A, u) & -2 \int_{\Omega \cap A^{(0)} \cap \partial A} \varphi\left(x, v_{A}\right) d \mathcal{H}^{1} \\
& -\int_{\Sigma \cap A^{(0)} \cap \partial A}\left(\phi\left(x, v_{A}\right)+\beta\right) d \mathcal{H}^{1}-\int_{\Sigma} \beta d \mathcal{H}^{1}
\end{aligned}
$$

admits a minimizer $(A, u)$ in every $\tau_{\mathcal{C}}^{\prime}$-closed subset of

$$
\mathcal{C}_{m}^{\prime}:=\left\{(A, u) \in \mathcal{C}: \text { A open, }|A|=\mathrm{v}, \text { and } A \cup \Sigma \in \mathcal{A}_{m}\right\}
$$

where $\left\{\left(A_{k}, u_{k}\right)\right\} \subset \mathcal{C}$ converges to $(A, u) \in \mathcal{C}$ in $\tau_{\mathcal{C}}^{\prime}$-sense if

$$
\begin{aligned}
& -\sup _{k \geqq 1} \mathcal{H}^{1}\left(\partial A_{k}\right)<\infty, \\
& -\mathbb{R}^{2} \backslash A_{k} \stackrel{\mathcal{K}}{\rightarrow} \mathbb{R}^{2} \backslash A, \\
& -u_{k} \rightarrow \text { u.e. in } \operatorname{Int}(A) \cup S .
\end{aligned}
$$

Remark 2.10. The sets $\mathcal{C}_{\text {subgraph }}$ and $\mathcal{C}_{\text {starshaped }}$ defined in Section 2.2 are $\tau_{\mathcal{C}}^{\prime}$-closed in $\mathcal{C}_{m}^{\prime}$ (see e.g., [31, Proposition 2.2]). In the thin-film setting, we define $\varphi$ and $\beta$ as $\varphi:=\gamma_{f}$ and

$$
\beta:=-\max \left\{\min \left\{\gamma_{f}, \gamma_{s}-\gamma_{f s}\right\},-\gamma_{f}\right\}
$$

where $\gamma_{f}, \gamma_{s}$, and $\gamma_{f s}$ denote the surface tensions of the film-vapor, substrate-vapor, and film-substrate interfaces, respectively. The energy $\mathcal{F}^{\prime}$ coincides (apart from the presence of delamination) with the thin-film energy in [22,23] in the case $\gamma_{f}, \gamma_{s}$, $\gamma_{f s}$ are constants, $\gamma_{s}-\gamma_{f s} \geqq 0, \gamma_{s}>0$, and $\gamma_{f}>0$. Therefore, Theorem 2.9 extends the existence results in [22,31] to all values of $\gamma_{s}$ and $\gamma_{s}-\gamma_{f s}$, as well as to anisotropic surface tensions and anisotropic elastic densities.

Remark 2.11. All the results contained in this subsection hold true with essentially the same proofs by replacing (H3) with the more general assumption

(H3') $W:(\Omega \cup S) \times \mathbb{M}_{\text {sym }}^{2 \times 2} \rightarrow[0, \infty)$ is a function such that $M \mapsto W(x, M)$ is convex for any $x \in \Omega \cup S$ and

$$
c^{\prime}|M|^{p} \leqq W(x, M) \leqq c^{\prime \prime}|M|^{p}+f(x)
$$

for some $p \geqq 2, c^{\prime \prime} \geqq c^{\prime}>0$ and $f \in L^{1}(\Omega \cup S)$. 


\section{Compactness}

In this section we prove Theorem 2.7. Convergence of sets with respect to the signed distance functions has the following compactness property.

Proposition 3.1. (Blaschke-type selection principle) For every sequence $\left\{A_{k}\right\}$ of subsets $\mathbb{R}^{2}$ there exist a subsequence $\left\{A_{k_{l}}\right\}$ and $A \subset \mathbb{R}^{2}$ such that $\operatorname{sdist}\left(\cdot, \partial A_{k_{l}}\right) \rightarrow$ $\operatorname{sdist}(\cdot, \partial A)$ locally uniformly in $\mathbb{R}^{2}$ as $l \rightarrow \infty$.

Proof. Without loss of generality we suppose $A_{k} \notin\left\{\mathbb{R}^{2}, \varnothing\right\}$. By the Blaschke selection principle [3, Theorem 6.1], there exists a not relabelled subsequence $\left\{A_{k}\right\}$ and a closed set $K \subset \mathbb{R}^{2}$ such that $\partial A_{k}$ converges to $K$ in the Kuratowski sense as $k \rightarrow \infty$. Notice that by Proposition A.1,

$$
\left|\operatorname{sdist}\left(\cdot, \partial A_{k}\right)\right| \rightarrow \operatorname{dist}(\cdot, K)
$$

locally uniformly as $k \rightarrow \infty$ since $\left|\operatorname{sdist}\left(\cdot, \partial A_{k}\right)\right|=\operatorname{dist}\left(\cdot, \partial A_{k}\right)$. As $\operatorname{sdist}\left(\cdot, \partial A_{k}\right)$ is 1-Lipschitz, by the Arzela-Ascoli Theorem, passing to a further not relabelled subsequence one can find $f: \mathbb{R}^{2} \rightarrow[-\infty,+\infty]$ such that

$$
\operatorname{sdist}\left(\cdot, \partial A_{k}\right) \rightarrow f
$$

locally uniformly in $\mathbb{R}^{2}$ as $k \rightarrow \infty$. By (3.1), $|f(\cdot)|=\operatorname{dist}(\cdot, K)$. Recall that $K$ may have nonempty interior. Fix a countable set $Q \subset \operatorname{Int}(K)$ dense in $\operatorname{Int}(K)$, and define

$$
A:=\{f<0\} \cup(\operatorname{Int}(\overline{\{f>0\}}) \cap \partial K) \cup Q .
$$

By construction, $\operatorname{Int}(A)=\{f<0\}, \bar{A}=\{f \leqq 0\} \cup K$ and $\partial A=\{f=0\}=K$.

Finally we show that

$$
f(x)=\operatorname{sdist}(x, \partial A) .
$$

If $x \in A$, by the definition of $A$ and $K, f(x) \leqq 0$ so that

$$
f(x)=-\operatorname{dist}(x, K)=-\operatorname{dist}(x, \partial A)=-\operatorname{dist}\left(x, \mathbb{R}^{2} \backslash A\right) .
$$

Analogously, if $x \notin A$, then $f(x) \geqq 0$ and hence

$$
f(x)=\operatorname{dist}(x, K)=\operatorname{dist}(x, \partial A)=\operatorname{dist}(x, A) .
$$

In general, the collection $\mathcal{A}$ is not closed under $\tau_{\mathcal{A}}$-convergence. Indeed, let $E:=\left\{x_{k}\right\}$ be a countable dense set in $B_{1}(0)$ and $E_{k}:=\left\{x_{1}, \ldots, x_{k}\right\} \in \mathcal{A}$. Then $\mathcal{H}^{1}\left(\partial E_{k}\right)=0$, and $E_{k} \stackrel{\tau_{\mathcal{A}}}{\rightarrow} E$ as $k \rightarrow \infty$, but $E \notin \mathcal{A}$ since $\partial E=\overline{B_{1}(0)}$. However, $\mathcal{A}_{m}$ is closed with respect to the $\tau_{\mathcal{A}}$-convergence.

Lemma 3.2. Let $A \subset \Omega$ and $\left\{A_{k}\right\} \subset \mathcal{A}_{m}$ be such that $A_{k} \stackrel{\tau_{\mathcal{A}}}{\rightarrow}$ A. Then: 
(a) $A \in \mathcal{A}_{m}$ and

$$
\mathcal{H}^{1}(\partial A) \leqq \liminf _{k \rightarrow \infty} \mathcal{H}^{1}\left(\partial A_{k}\right)
$$

(b) $A_{k} \rightarrow A$ in $L^{1}\left(\mathbb{R}^{2}\right)$ as $k \rightarrow \infty$.

Proof. (a) By Remark 2.2, $\partial A_{k} \stackrel{\mathcal{K}}{\rightarrow} \partial A$ as $k \rightarrow \infty$. Thus, by [36, Theorem 2.1] $\partial A$ has at most $m$-connected components, and (3.2) holds.

(b) As $\partial A_{k} \stackrel{\mathcal{K}}{\rightarrow} \partial A$, for any $x \in \operatorname{Int}(A)$ resp. $x \in \mathbb{R}^{2} \backslash \bar{A}$, there exists $k_{x}>0$ such that $x \in A_{k}$ resp. $x \in \mathbb{R}^{2} \backslash \overline{A_{k}}$ for all $k>k_{x}$. Finally, by (3.2), $|\partial A|=0$, and therefore,

$$
\chi_{A_{k}} \rightarrow \chi_{A} \quad \text { a.e. } x \in \mathbb{R}^{2} \text {. }
$$

Now (b) follows from the uniform boundedness of $\left\{A_{k}\right\}$ and the Dominated Convergence Theorem.

Furthermore, sequences $\left\{A_{k}\right\} \subset \mathcal{A}_{m}$ with equibounded boundary lengths are compact with respect to the $\tau_{\mathcal{A}}$-convergence.

Proposition 3.3. (Compactness of $\mathcal{A}_{m}$ ) Suppose that $\left\{A_{k}\right\} \subset \mathcal{A}_{m}$ is such that

$$
\sup _{k \geqq 1} \mathcal{H}^{1}\left(\partial A_{k}\right)<\infty .
$$

Then there exists a subsequence $\left\{A_{k_{l}}\right\}$ and $A \in \mathcal{A}_{m}$ such that $\mathcal{H}^{1}(\partial A)<\infty$ and $\operatorname{sdist}\left(\cdot, \partial A_{k_{l}}\right) \rightarrow \operatorname{sdist}(\cdot, \partial A)$ locally uniformly in $\mathbb{R}^{2}$ as $l \rightarrow \infty$.

Proof. By Proposition 3.1 there exists a not relabelled subsequence $\left\{A_{k}\right\}$ and a set $A$ such that $\partial A_{k} \stackrel{\mathcal{K}}{\rightarrow} \partial A$ and $\operatorname{sdist}\left(\cdot, \partial A_{k}\right) \rightarrow \operatorname{sdist}(\cdot, \partial A)$ locally uniformly in $\mathbb{R}^{2}$ as $k \rightarrow \infty$. By Lemma 3.2, $A \in \mathcal{A}_{m}$ and $\mathcal{H}^{1}(\partial A)<\infty$.

Proposition 3.4. Let $\left\{A_{k}\right\} \subset \mathcal{A}_{m}$ be such that $A_{k} \stackrel{\tau_{\mathcal{A}}}{\rightarrow} A$ as $k \rightarrow \infty$. Suppose that

$$
\operatorname{Int}(A)=\bigcup_{h \in I} E_{h}, \quad F=\bigcup_{i \in I_{1}} E_{i} \quad \text { and } \quad G=\bigcup_{j \in I_{2}} E_{j} \text {, }
$$

where $E_{h}$ are disjoint connected components of $\operatorname{Int}(A), I_{1}$ and $I_{2}$ are disjoint finite subsets of $I$. Then there exist a subsequence $\left\{A_{k_{l}}\right\}$ and a sequence $\left\{\gamma_{l}\right\}$ of $\mathcal{H}^{1}$-rectifiable sets in $\mathbb{R}^{2}$ such that

(a) $\gamma_{l} \subset \operatorname{Int}\left(A_{k_{l}}\right)$ and $\lim _{l \rightarrow \infty} \mathcal{H}^{1}\left(\gamma_{l}\right)=0$;

(b) $\operatorname{sdist}\left(\cdot, \partial\left(A_{k_{l}} \backslash \gamma_{l}\right)\right) \rightarrow \operatorname{sdist}(\cdot, \partial A)$ as $l \rightarrow \infty$ locally uniformly in $\mathbb{R}^{2}$;

(c) for any connected open sets $D^{\prime} \subset \subset F$ and $D^{\prime \prime} \subset \subset G$ there exists $l^{\prime}$ such that $D^{\prime}$ and $D^{\prime \prime}$ belong to different connected components of $\operatorname{Int}\left(A_{k_{l}} \backslash \gamma_{l}\right)$ for any $l>l^{\prime}$. 
We postpone the proof until after the next lemma. Before this, we need to introduce some notation. Let $n_{0}>1$ be such that $E_{h} \cap\left\{\operatorname{dist}(\cdot, \partial A)>\frac{1}{n}\right\} \neq \emptyset$ for every $h \in I_{1} \cup I_{2}$ and $n>n_{0}$. Given $h \in I_{1} \cup I_{2}$, let $\left\{E_{h}^{n}\right\}_{n>n_{0}}$ be an increasing sequence of connected open sets satisfying $E_{h} \cap\left\{\operatorname{dist}(\cdot, \partial A)>\frac{1}{n}\right\} \subseteq E_{h}^{n} \subset \subset E_{h}$ and

$$
E_{h}=\bigcup_{n \geqq n_{0}} E_{h}^{n} .
$$

By the $s d i s t$-convergence and the finiteness of $I_{1} \cup I_{2}$, for any $n \geqq n_{0}$ there exist $k_{n}^{0}>0$ such that $E_{h}^{n} \subset \subset \operatorname{Int}\left(A_{k}\right)$ for all $k>k_{n}^{0}$ and $h \in I_{1} \cup I_{2}$. Let

$$
2 d_{n}:=\min _{i \in I_{1}, j \in I_{2}}\left\{\operatorname{dist}\left(E_{i}^{n}, E_{j}^{n}\right), \operatorname{dist}\left(E_{i}^{n}, \partial A\right), \operatorname{dist}\left(E_{j}^{n}, \partial A\right)\right\} .
$$

Note that $0<d_{n}<\frac{1}{2 n}$.

The idea of the proof of Proposition 3.4 is to "partition" the connecting components of $\operatorname{Int}\left(A_{k}\right)$ which in the limit break down into connected components $\left\{E_{h}\right\}_{h \in I^{\prime}}$ of $\operatorname{Int}(A)$ such that $I^{\prime} \cap I_{1} \neq \varnothing$ and $I^{\prime} \cap I_{2} \neq \varnothing$, for example in the case of neckpinches. More precisely, we cut out at most $m$-circles from $\operatorname{Int}\left(A_{k}\right)$ such that for any $n>n_{0}$, for all sufficiently large $k$ (depending only on $n$ ), any curve $\gamma \subset \operatorname{Int}\left(A_{k}\right)$ connecting a point of $E_{i}^{n}, i \in I_{1}$, to a point of $E_{j}^{n}, j \in I_{2}$, intersects at least one of these circles. The following lemma consists in performing this argument for fixed $i \in I_{1}$ and $j \in I_{2}$ :

Lemma 3.5. Under the assumptions of Proposition 3.4, let $i \in I_{1}, j \in I_{2}$, and $n>n_{0}$ be such that the set

$$
\begin{array}{r}
Y=Y_{i j}^{n}:=\left\{k \in \mathbb{N}: \exists D_{k} \subset \subset \operatorname{Int}\left(A_{k}\right)\right. \text { closed, connected, } \\
\text { and such that } \left.D_{k} \cap E_{i}^{n}, D_{k} \cap E_{j}^{n} \neq \varnothing\right\}
\end{array}
$$

is infinite. Then, there exists $k_{n}^{i j}>k_{n}^{0}$ such that for any $k \in Y$ with $k>k_{n}^{i j}$ there exists a collection $\left\{B_{r_{k}^{l}}\left(z_{k}^{l}\right)\right\}_{l}$ of at most $m$ balls contained in $A_{k}$ such that $r_{k}^{l}<d_{n}$ and any curve $\gamma \subset \subset \operatorname{Int}\left(A_{k}\right)$, connecting a point of $E_{i}^{n}$ to a point of $E_{j}^{n}$, intersects at least one of $B_{r_{k}^{l}}\left(z_{k}^{l}\right)$.

Proof. We divide the proof into four steps.

Step 1: for any $k \in Y$, let $C_{k} \subset \subset \operatorname{Int}\left(A_{k}\right)$ be any closed connected set intersecting both $E_{i}^{n_{0}}$ and $E_{j}^{n_{0}}$. Then

$$
\lim _{k \in Y, k \rightarrow \infty} \operatorname{dist}\left(C_{k}, \partial A_{k}\right)=0 .
$$

By contradiction, assume that there exists $\epsilon>0$ such that

$$
\operatorname{dist}\left(C_{k}, \partial A_{k}\right) \geqq \epsilon
$$

for infinitely many $k \in Y$. By the Kuratowski-compactness of closed sets there exist a closed connected set $C$ and a not relabelled subsequence $\left\{C_{k}\right\}_{k \in Y}$ satisfying 
(3.6) for all $k \in Y$ such that $C_{k} \stackrel{K}{\rightarrow} C$ as $k \rightarrow \infty$. Since $A_{k} \stackrel{\tau_{\mathcal{A}}}{\rightarrow} A$, in view of Remark $2.2 \partial A_{k} \stackrel{K}{\rightarrow} \partial A$ and $D \subset A$. Let $x \in C$ and $y \in \partial A$ be such that $|x-y|=\operatorname{dist}(C, \partial A)$. Then by the definition of the Kuratowski convergence, there exist sequences $x_{k} \in C_{k}$ and $y_{k} \in \partial A_{k}$ such that $x_{k} \rightarrow x$ and $y_{k} \rightarrow y$. Since $\left|x_{k}-y_{k}\right| \geqq \operatorname{dist}\left(C_{k}, \partial A_{k}\right) \geqq \epsilon$, it follows that

$$
\operatorname{dist}(C, \partial A)=|x-y|=\lim _{k \rightarrow \infty}\left|x_{k}-y_{k}\right| \geqq \epsilon .
$$

Thus, $C \subset \subset \operatorname{Int}(A)$. In particular, (3.7) implies that the non-empty connected open set $\left\{\operatorname{dist}(\cdot, C)<\frac{\epsilon}{4}\right\}$ is compactly contained in $\operatorname{Int}(A)$ and intersects both $E_{i}^{n_{0}}$ and $E_{j}^{n}$ so that $E_{i}^{n} \cup\left\{\operatorname{dist}(\cdot, C)<\frac{\epsilon}{4}\right\} \cup E_{j}^{n} \subset \operatorname{Int}(A)$ is connected. But this is a contradiction since $E_{i}^{n}$ and $E_{j}^{n}$ belong to different connected components of $\operatorname{Int}(A)$.

Step 2: for every $k \in Y$ there exists a path-connected closed set $L_{k} \subset \subset \operatorname{Int}\left(A_{k}\right)$ intersecting both $E_{i}^{n}$ and $E_{j}^{n}$ such that

$$
\operatorname{dist}\left(L_{k}, \partial A_{k}\right)=\delta_{k}:=\sup \operatorname{dist}\left(D, \partial A_{k}\right) \text {, }
$$

where sup is taken over all closed connected sets $D \subset \subset \operatorname{Int}\left(A_{k}\right)$, intersecting both $E_{i}^{n_{0}}$ and $E_{j}^{n_{0}}$ (such sets exist by definition of $Y$ ). Moreover, there exists $k_{n}^{1}>0$ such that $L_{k}$ contains $E_{i}^{n} \cup E_{j}^{n}$ and $\delta_{k}<d_{n}$ for any $k>k_{n}^{1}$.

Indeed, in view of the Kuratowski-compactness of closed sets and from the Kuratowski-continuity of $\operatorname{dist}\left(\cdot, \partial A_{k}\right)$, (3.8) has a maximizer $L_{k}^{\prime}$. Applying Step 1 with $A_{k}$ and $C_{k}=L_{k}^{\prime}$, we get $\delta_{k} \rightarrow 0$ as $k \rightarrow \infty$. Let $L_{k}$ be the connected component of $\left\{\operatorname{dist}\left(\cdot, \partial A_{k}\right) \geqq \delta_{k}\right\}$ containing $L_{k}^{\prime}$. Since $E_{i}^{n} \cup E_{j}^{n} \subset \subset \operatorname{Int}(A)$, the sdist-convergence and Remark 2.2, $E_{i}^{n} \cup E_{j}^{n} \subset \subset \operatorname{Int}\left(A_{k}\right)$ for all large $k$. More precisely, by the definition (3.4) of $d_{n}$, there exists $\bar{k}_{n}^{1}>0$ such that

$$
\min \left\{\operatorname{dist}\left(E_{i}^{n}, \partial A_{k}\right), \operatorname{dist}\left(E_{j}^{n}, \partial A_{k}\right)\right\} \geqq d_{n}
$$

for all $k>\bar{k}_{n}^{1}$. By construction, $\operatorname{dist}\left(L_{k}, \partial A_{k}\right)=\delta_{k}$, and since $\delta_{k} \rightarrow 0$, there exists $k_{n}^{1}>\bar{k}_{n}^{1}$ such that $\delta_{k}<d_{n}$ for any $k \geqq k_{n}^{1}$. Note that by (3.9) for such $k$ we have also $E_{i}^{n} \cup E_{j}^{n} \subset L_{k}$.

Let us show that $L_{k}$ is also path-connected. Indeed, given $x \in L_{k}$, consider the ball $B_{r}(x)$ for small $r<\delta_{k}$. Then $L_{k} \cap B_{r}(x)$ is path-connected, otherwise there would exist a curve in $B_{r}(x)$ with endpoints in $L_{k}$ containing a point $z \in B_{r}(x) \backslash L_{k}$ such that $\operatorname{dist}\left(z, \partial A_{k}\right)>\delta_{k}$ contradicting to the definition of $L_{k}$. Thus, $L_{k}$ is locally path-connected. Now the compactness and the connectedness of $L_{k}$ imply its path-connectedness.

Step 3: given $x \in E_{i}^{n_{0}}$ and $y \in E_{j}^{n_{0}}$, let $\gamma_{k} \subset L_{k}$ be a curve connecting $x$ to $y$. Then for any $k>k_{\epsilon}^{0}$ there exists $z_{k} \in \gamma_{k} \backslash \overline{E_{i}^{n_{0}} \cup E_{j}^{n_{0}}}$ such that any curve $\gamma \subset \subset \operatorname{Int}\left(A_{k}\right)$ homotopic in $\operatorname{Int}\left(A_{k}\right)$ to $\gamma_{k}$ (with same endpoints) intersects the ball $B_{\delta_{k}}\left(z_{k}\right)$.

Indeed, otherwise slightly perturbing the curve $\gamma_{k}$ around the points of the compact set $\gamma^{\prime}:=\left\{x \in \gamma_{k}: \operatorname{dist}\left(x, \partial A_{k}\right)=\delta_{k}\right\}$ we would get a new curve $\tilde{\gamma}_{k} \subset \subset \operatorname{Int}\left(A_{k}\right)$ connecting $x$ to $y$ for which $\operatorname{dist}\left(x, \gamma_{k}\right)>\delta_{k}$ for all $x \in \tilde{\gamma}_{k}$. 
Now the compactness of $\tilde{\gamma}_{k}$ implies $\operatorname{dist}\left(\tilde{\gamma}_{k}, \partial A_{k}\right)>\delta_{k}$, which contradicts to the definition (3.8) of $L_{k}$.

Step 4: now we prove the lemma.

Applying Steps 1-3 with $A_{k}$, we find an integer $k_{n}^{1}>k_{n}^{0}$, a curve $\gamma_{k}^{1}$ connecting a point of $E_{i}^{n_{0}}$ to a point $E_{j}^{n_{0}}$ such that

$$
\operatorname{dist}\left(\gamma_{k}^{1}, \partial A_{k}\right)=r_{k}^{1}=\sup \operatorname{dist}\left(D, \partial A_{k}\right)<d_{n}
$$

where sup is taken over all connected and closed $D \subset \subset \operatorname{Int}\left(A_{k}\right)$ intersecting both $E_{i}^{n_{0}}$ and $E_{j}^{n_{0}}$, and a ball $B_{r_{k}^{1}}\left(z_{k}^{1}\right) \subset A_{k}$ with $z_{k}^{1} \in \gamma_{k}^{1}$ such that any curve $\gamma \subset \subset \operatorname{Int}\left(A_{k}\right)$ homotopic to $\gamma_{k}^{1}$ intersects $B_{r_{k}^{1}}\left(z_{k}^{1}\right)$ for any $k \in Y$ with $k>k_{n}^{1}$.

For $k \in Y$ with $k>k_{n}^{1}$ set

$$
A_{k}^{1}:=A_{k} \backslash\left(\operatorname{Int}\left(A_{k}\right) \cap \partial B_{r_{k}^{1}}\left(z_{k}^{1}\right)\right) .
$$

Now consider the set $Y_{1}$ of all $k \in Y$ for which there exists a closed connected set $C_{k} \subset \subset \operatorname{Int}\left(A_{k}\right)$ intersecting both $E_{i}^{n_{0}}$ and $E_{j}^{n_{0}}$. If $Y_{1}$ is finite, we set $k_{n}^{i j}:=$ $\max \left\{\max Y_{1}, k_{n}^{1}\right\}$ and we are done.

Assume that $Y_{1}$ is infinite. Note that for any $k \in Y_{1}, \partial B_{r_{k}^{1}}\left(z_{k}^{1}\right)$ touches at least two different connected components of $\partial A_{k}$ and thus, $A_{k}^{1} \in \mathcal{A}_{m-1}$. Applying Steps 1-3 with $A_{k}^{1}$ and $Y_{1}$, we find an integer $k_{n}^{2}>k_{n}^{1}$, a curve $\gamma_{k}^{2}$ connecting a point of $E_{i}^{n_{0}}$ to a point $E_{j}^{n_{0}}$ such that

$$
\operatorname{dist}\left(\gamma_{k}^{2}, \partial A_{k}^{1}\right)=r_{k}^{2}=\sup \operatorname{dist}\left(D, \partial A_{k}^{1}\right)
$$

where sup is taken over all connected and closed $D \subset \subset \operatorname{Int}\left(A_{k}^{1}\right)$ intersecting both $E_{i}^{n_{0}}$ and $E_{j}^{n_{0}}$, and a ball $B_{r_{k}^{2}}\left(z_{k}^{2}\right) \subset A_{k}^{1}$ with $z_{k}^{2} \in \gamma_{k}^{2}$ such that any curve $\gamma \subset \subset \operatorname{Int}\left(A_{k}\right)$ homotopic to $\gamma_{k}^{2}$ intersects $B_{r_{k}^{2}}\left(z_{k}^{2}\right)$ for any $k \in Y_{1}$ with $k>k_{n}^{2}$. By (3.10), $r_{k}^{1} \geqq r_{k}^{2}$.

For $k \in Y_{1}$ with $k>k_{n}^{2}$ set

$$
A_{k}^{2}:=A_{k} \backslash\left(\operatorname{Int}\left(A_{k}\right) \cap\left(\partial B_{r_{k}^{1}}\left(z_{k}^{1}\right) \cup \partial B_{r_{k}^{2}}\left(z_{k}^{2}\right)\right)\right)
$$

and consider the set $Y_{2}$ of all $k \in Y_{1}$ for which there exists a closed connected set $C_{k} \subset \subset \operatorname{Int}\left(A_{k}^{2}\right)$ intersecting both $E_{i}^{n_{0}}$ and $E_{j}^{n_{0}}$. Note that $Y_{2}$ is finite, setting $k_{n}^{i j}:=\max \left\{\max Y_{2}, k_{n}^{2}\right\}$ and we are done. If $Y_{2}$ is infinite, then $A_{k}^{2} \in \mathcal{A}_{m-2}$, and we repeat the same procedure above. After at most $m$ steps we obtain $k_{n}^{i j}>k_{n}^{0}$ such that for any $k>k_{n}^{i j}$ there is a collection $\left\{B_{r_{k}^{l}}\left(z_{k}^{l}\right)\right\}$ of at most $m$ balls, which satisfy the assertion of the lemma.

The assertions of Proposition 3.4 follow by applying Lemma 3.5 with all pairs $(i, j) \in I_{1} \times I_{2}$. 
Proof of Proposition 3.4. Given $i \in I_{1}, j \in I_{2}$ and $n>n_{0}$, let $Y_{i j}^{n}$ be given by (3.5). If $Y_{i j}^{n}$ is infinite, let $k_{n}^{i j}$ be given by Lemma 3.5, otherwise set $k_{n}^{i j}:=1+\max Y_{i j}^{n}$. Let $k_{n}:=1+\max _{i, j} k_{n}^{i j}$ and

$$
\gamma_{n}^{i j}:= \begin{cases}\operatorname{Int}\left(A_{k_{n}}\right) \cap \bigcup_{l} \partial B_{r_{k_{n}}^{l}}^{i j}\left(z_{k_{n}}^{l}\right), & \text { if } k_{n} \in Y_{i j}^{n}, \\ \varnothing, & \text { if } k_{n} \notin Y_{i j}^{n},\end{cases}
$$

where $\left\{B_{r_{k}^{l}}^{i j}\left(z_{k}^{l}\right)\right\}$ is the collection of balls given by Lemma 3.5. Without loss of generality we assume that $\left\{k_{n}\right\}_{n}$ is strictly increasing and set

$$
\gamma_{n}:=\bigcup_{i, j} \gamma_{n}^{i j}
$$

Being a union of at most $N_{1} N_{2} m$ circles, $\gamma_{n}$ is $\mathcal{H}^{1}$-rectifiable; here $N_{i}$ is the cardinality of $I_{i}$. By Lemma 3.5,

$$
\mathcal{H}^{1}\left(\gamma_{n}\right) \leqq \sum_{i, j, l} \mathcal{H}^{1}\left(\partial B_{r_{k_{n}}^{l}}^{i j}\left(z_{k_{n}}^{l}\right)\right) \leqq 2 \pi N_{1} N_{2} m d_{n} .
$$

Then $\lim _{n \rightarrow \infty} \mathcal{H}^{1}\left(\gamma_{n}\right)=0$ and therefore, $\gamma_{n}$ converges in the Kuratowski sense to at most $N_{1} N_{2} m$ points on $\partial A$.

We claim that the sequences $\left\{A_{k_{n}}\right\}$ and $\left\{\gamma_{n}\right\}$ satisfy assertions (a)-(c). Indeed, by (3.11), $\left\{\gamma_{n}\right\}$ satisfy (a). Since $\gamma_{n}$ converges to at most $N_{1} N_{2} m$ points on $\partial A$ in the Kuratowski sense, (b) follows. To prove (c), we take any connected open sets $D^{\prime} \subset \subset E$ and $D^{\prime \prime} \subset \subset F$. By connectedness and the definitions of $E_{h}$ and $E_{h}^{n}$, there exist $i \in I_{1}$ and $j \in I_{2}$ and $\bar{n}>n_{0}$ such that $D^{\prime} \subset \subset E_{i}^{n}$ and $D^{\prime \prime} \subset \subset E_{j}^{n}$ for all $n>\bar{n}$. By the construction of $\gamma_{n}$, the sets $E_{i}^{n}$ and $E_{j}^{n}$ (and hence, $D^{\prime}$ and $D^{\prime \prime}$ ) belong to different connected components of $\operatorname{Int}\left(A_{k_{n}}\right) \backslash \gamma_{n}$ for all $n>\bar{n}$.

By inductively applying Proposition 3.4 and by means of a diagonal argument we modify a sequence $\left\{A_{k}\right\} \tau_{\mathcal{A}}$-converging to a set $A$ into a sequence $\left\{B_{k}\right\}$ with same $\tau_{\mathcal{A}}$-limit and whose (open) connected components "vanish" or "converge to the corresponding" connected components of $A$. This construction will be used in Step 1 of the proof of Theorem 2.7. We notice here that if $S=\emptyset$, then the sequence $\left\{D_{n}\right\}$ from Theorem 2.7 coincides with the sequence $\left\{B_{n}\right\}$. Actually, if $S=\emptyset$, it would be enough to take $D_{n}=\widetilde{B}_{n}$, where $\widetilde{B}_{n}$ is constructed in the Step 1 of the proof of the next proposition, since in this case we do not need properties (e) and (f) of the statement of the next proposition.

Proposition 3.6. Let $A \in \mathcal{A}_{m}$ and $\left\{A_{k}\right\} \subset \mathcal{A}_{m}$ be such that $\operatorname{sdist}\left(\cdot, \partial A_{k}\right) \rightarrow$ sdist $(\cdot, \partial A)$ locally uniformly in $\mathbb{R}^{2}$. Then there exist a subsequence $\left\{A_{k_{l}}\right\}$ and a sequence $\left\{B_{l}\right\} \subset \mathcal{A}_{m}$ such that

(a) $\partial A_{k_{l}} \subset \partial B_{l}$ and $\lim _{l \rightarrow \infty} \mathcal{H}^{1}\left(\partial B_{l} \backslash \partial A_{k_{l}}\right)=0$;

(b) $\operatorname{sdist}\left(\cdot, \partial B_{l}\right) \rightarrow \operatorname{sdist}(\cdot, \partial A)$ locally uniformly in $\mathbb{R}^{2}$; 
(c) if $\left\{E_{i}\right\}$ is the set of all connected components of $\operatorname{Int}(A)$, we can choose a subfamily $\left\{E_{i}^{l}\right\}$ of connected components of $\operatorname{Int}\left(B_{l}\right)$ such that for any $G \subset \subset E_{i}$ there exists $l_{i, G}>0$ with $G \subset \subset E_{i}^{l}$ for every $l>l_{i, G}$;

(d) $\left|B_{l}\right|=\left|A_{k_{l}}\right|$ for every $l \geqq 1$;

(e)

$$
\lim _{l \rightarrow \infty} \sup _{x \in E_{i}^{l} \backslash E_{i}} \operatorname{dist}\left(x, E_{i}\right)=0
$$

and

$$
\lim _{l \rightarrow \infty} \mathcal{H}^{1}\left(\partial \Omega \cap\left(\partial E_{i}^{l} \backslash \partial E_{i}\right)\right)=0
$$

(f) the boundary of every connected component of $\operatorname{Int}\left(B_{l}\right) \backslash \bigcup_{i} E_{i}^{l}$ intersects the boundary of at most one connected component of $S$.

Proof. Given $N, n \geqq 1$, we define the index set $I_{n}^{N}$ by

$$
I_{n}^{N}:=\left\{i>N: E_{i} \cap\left\{\operatorname{dist}(\cdot, \partial A)>\frac{1}{n}\right\} \neq \emptyset\right\} .
$$

We notice that $I_{n}^{N}$ is finite since $A$ is bounded.

Step 1: Construction of $\left\{\widetilde{B}_{l}\right\}$ and $\left\{A_{k_{l}}\right\}$ satisfying $(a)-(d)$. This is done by using Proposition 3.4 iteratively in $N \in \mathbb{N}$ and a diagonal argument.

Substep 1: Base of iteration. By Proposition 3.4 applied with $\left\{A_{k}\right\}_{k \in Y^{0}}$ with $Y^{0}:=\mathbb{N}, I_{1}=\{1\}$, and $I_{2}=I_{n}^{1}$ inductively with respect to $n \in \mathbb{N}$, we find a decreasing sequence $Y^{0} \supset Y^{1} \supset \ldots$ of infinite subsets of $\mathbb{N}$ such that for the subsequence $\left\{A_{k}\right\}_{k \in Y^{n}}$ there exists a sequence $\left\{\gamma_{k}^{n}\right\}_{k \in Y^{n}}$ of $\mathcal{H}^{1}$-rectifiable sets such that for any $n \geqq 1$ :

- $\gamma_{k}^{n} \subset \operatorname{Int}\left(A_{k}\right)$ for any $k \in Y^{n}$ and $\lim _{k \in Y^{n}, k \rightarrow \infty} \mathcal{H}^{1}\left(\gamma_{k}^{n}\right)=0$;

- for any connected open sets $D \subset \subset E_{1}$ and $D^{\prime} \subset \subset \cup_{j \in I_{n}^{1}} E_{j}$ there exists $k^{\prime}>0$ such that $D$ and $D^{\prime}$ belong to different connected components of $A_{k} \backslash \gamma_{k}^{n}$ for any $k \in Y^{n}$ with $k>k^{\prime}$;

- $\operatorname{sdist}\left(\cdot, \partial\left(A_{k} \backslash \gamma_{k}^{n}\right)\right) \rightarrow \operatorname{sdist}(\cdot, \partial A)$ as $Y^{n} \ni k \rightarrow \infty$ locally uniformly in $\mathbb{R}^{2}$.

Then by a diagonal argument, we choose an increasing sequence $n \in \mathbb{N} \mapsto k_{n}^{1} \in Y^{n}$ such that

$$
\widetilde{B}_{1, n}:=A_{k_{n}} \backslash \gamma_{k_{n}^{1}}^{n}, \quad n \in \mathbb{N}
$$

satisfies

$a_{1 n}: \partial A_{k_{n}} \subset \partial \widetilde{B}_{1, n}$ and $\mathcal{H}^{1}\left(\partial \widetilde{B}_{1, n} \backslash \partial A_{k_{n}}\right)=\mathcal{H}^{1}\left(\gamma_{k_{n}^{1}}^{n}\right)<2^{-n}$ for any $n \geqq 1$;

$b_{1 n}: \operatorname{sdist}\left(\cdot, \partial \widetilde{B}_{1, n}\right) \rightarrow \operatorname{sdist}(\cdot, \partial A)$ as $n \rightarrow \infty$ locally uniformly in $\mathbb{R}^{2}$;

$c_{1 n}$ : for any connected open set $D \subset \subset E_{1}$ there exist $n_{D}^{1}>1$ and a unique connected component denoted by $E_{1}^{1, n}$ of $\operatorname{Int}\left(\widetilde{B}_{1, n}\right)$ such that $D \subset \subset E_{1}^{1, n}$ for all $n>n_{D}^{1}$. 
Substep 2: Iterative argument. Repeating Substep 1 and applying Proposition 3.4 inductively in $N=1,2, \ldots$, with $A_{k}:=\widetilde{B}_{N, k}, I_{1}:=\{1, \ldots, N\}$ and $I_{2}:=I_{n}^{N}$ for $n \in \mathbb{N}$, we obtain $\left\{\widetilde{B}_{N+1, n}\right\}_{n} \subset \mathcal{A}_{m}$ and and increasing sequence $n \in \mathbb{N} \mapsto$ $k_{n}^{N+1}$ with $\left\{k_{n}^{N}\right\}_{n} \supset\left\{k_{n}^{N+1}\right\}_{n}$ such that for any $N \geqq 1$ :

$a_{N n}: \partial A_{k_{n}^{N}} \subset \partial \widetilde{B}_{N, n}, \partial \widetilde{B}_{N, n} \subset \partial \widetilde{B}_{N+1, n}$ and $\mathcal{H}^{1}\left(\partial \widetilde{B}_{N+1, n} \backslash \partial \widetilde{B}_{N, n}\right)<$ $2^{-(N+1) n}$ for any $n \geqq 1$;

$b_{N n}: \operatorname{sdist}\left(\cdot, \partial \widetilde{B}_{N, n}\right) \rightarrow \operatorname{sdist}(\cdot, \partial A)$ as $n \rightarrow \infty$ locally uniformly in $\mathbb{R}^{2}$;

$c_{N n}$ : for any connected open set $D \subset \subset E_{i}$ for some $i \in\{1, \ldots, N\}$ there exist $n_{D}^{i}>1$ and a unique connected component denoted by $E_{i}^{N, n}$ of $\operatorname{Int}\left(\widetilde{B}_{N, n}\right)$ such that $D \subset \subset E_{i}^{N, n}$ for all $n>n_{D}^{i}$.

By condition $b_{N n}$ in Substep 2 and by the uniform boundedness of $\left\{\widetilde{B}_{N, n}\right\}$, there exists an increasing sequence $N \in \mathbb{N} \mapsto n_{N} \in \mathbb{N}$ such that the sequence $\widetilde{B}_{N}:=\widetilde{B}_{N, n_{N}}$ satisfies sdist $\left(\cdot, \partial \widetilde{B}_{N}\right) \rightarrow \operatorname{sdist}(\cdot, \partial A)$ as $N \rightarrow \infty$ locally uniformly in $\mathbb{R}^{2}$. By condition $a_{N n_{N}}$ of Substep 2,

$$
\partial A_{k_{n_{N}}^{N}} \subset \partial \widetilde{B}_{1, n_{N}} \subset \ldots \subset \partial \widetilde{B}_{N, n_{N}}=\partial \widetilde{B}_{N}
$$

and

$$
\mathcal{H}^{1}\left(\partial \widetilde{B}_{N} \backslash \partial A_{k_{n_{N}}^{N}}\right) \leqq \sum_{i=1}^{N} \mathcal{H}^{1}\left(\partial \widetilde{B}_{i, n_{N}} \backslash \partial \widetilde{B}_{i-1, n_{N}}\right) \leqq \sum_{i=1}^{N} 2^{-i n_{N}}<2^{1-n_{N}}
$$

where $\widetilde{B}_{0, n_{N}}:=A_{k_{n_{N}}^{N}}$.

Furthermore, given $i \in \mathbb{N}$, if $D \subset \subset E_{i}$ is any connected open set, then by condition $c_{N n_{N}}$, there exists a unique connected component $\widetilde{E}_{i}^{N}:=E_{i}^{N, n_{N}}$ of $\operatorname{Int}\left(\widetilde{B}_{N}\right)$ such that $D \subset \subset E_{i}^{N}$ for all sufficiently large $N$ (depending only $D$ and $i$ ). Moreover, it is clear that $\left|\widetilde{B}_{N}\right|=\left|A_{k_{n_{N}}^{N}}\right|$ for any $N$. Hence, the sequence $\left\{\widetilde{B}_{N}\right\}_{N}$ and the subsequence $\left\{A_{k_{n_{N}}^{N}}\right\}_{N}$ satisfy assertions (a)-(d).

Step 2: Construction of $\left\{\widehat{B}_{l}\right\}$ and $\left\{A_{k_{l}}\right\}$ satisfying $(a)-(e)$. Notice that $\operatorname{Int}\left(\widetilde{B}_{N}\right) \subset$ $\operatorname{Int}\left(A_{k_{n_{N}}^{N}}\right)$ and by $\widetilde{B}_{N} \stackrel{\tau_{\mathcal{A}}}{\rightarrow} A$ and Lemma $3.2(\mathrm{~b}), \lim _{N \rightarrow \infty}\left|\widetilde{B}_{N} \Delta A\right| \rightarrow 0$. In particular, for any $i$,

$$
\lim _{N \rightarrow \infty}\left|\widetilde{E}_{i}^{N} \Delta E_{i}\right|=0
$$

By the Area Formula applied with $\operatorname{dist}\left(\cdot, E_{i}\right)$ we have

$$
\left|\widetilde{E}_{i}^{N} \backslash E_{i}\right|=\int_{0}^{\infty} \mathcal{H}^{1}\left(\left(\widetilde{E}_{i}^{N} \backslash E_{i}\right) \cap\left\{\operatorname{dist}\left(\cdot, E_{i}\right)=t\right\}\right) \mathrm{d} t
$$

for any $i$. From this, (3.12) and a diagonal argument, there exists a not relabelled subsequence $\left\{\widetilde{B}_{N}\right\}$ for which

$$
\lim _{N \rightarrow \infty} \mathcal{H}^{1}\left(\left(\widetilde{E}_{i}^{N} \backslash E_{i}\right) \cap\left\{\operatorname{dist}\left(\cdot, E_{i}\right)=t\right\}\right)=0
$$


for any $i$ and a.e. $t>0$. Thus, we can choose $t_{S} \searrow 0$ for which

$$
\lim _{N \rightarrow \infty} \mathcal{H}^{1}\left(\left(\widetilde{E}_{i}^{N} \backslash E_{i}\right) \cap\left\{\operatorname{dist}\left(\cdot, E_{i}\right)=t_{s}\right\}\right)=0
$$

for any $s \in \mathbb{N}$ and $i$, and thus, by a diagonal argument we find a further subsequence $\left\{\widetilde{B}_{N_{s}}\right\}_{s \in \mathbb{N}}$ such that

$$
\mathcal{H}^{1}\left(\left(\widetilde{E}_{i}^{N_{s}} \backslash E_{i}\right) \cap\left\{\operatorname{dist}\left(\cdot, E_{i}\right)=t_{s}\right\}\right)<2^{-i s}
$$

for any $i$ and $s$. Let $\zeta_{i}^{s}:=\left(\widetilde{E}_{i}^{N_{s}} \backslash E_{i}\right) \cap\left\{\operatorname{dist}\left(\cdot, E_{i}\right)=t_{s}\right\}$, and let

$$
\widehat{B}_{s}:=\widetilde{B}_{N_{s}} \backslash \zeta_{s}
$$

where $\zeta_{s}:=\bigcup_{i} \zeta_{i}^{s}$. Note that $\zeta_{s}$ is $\mathcal{H}^{1}$-rectifiable and by $(3.13), \mathcal{H}^{1}\left(\zeta_{s}\right) \leqq 2^{1-s}$.

Denote by $\widehat{E}_{i}^{s}$ the connected component of $\widehat{B}_{s}$ satisfying $\widehat{E}_{i}^{s} \subset \widetilde{E}_{i}^{N_{s}}$ and $\widehat{E}_{i}^{s} \cap E_{i} \neq$ $\emptyset$. By construction, $\sup _{x \in \widehat{E}_{i}^{s} \backslash E_{i}} \operatorname{dist}\left(x, E_{i}\right) \leqq t_{s}$, thus,

$$
\limsup _{s \rightarrow \infty} \sup _{x \in \widehat{E}_{i}^{s} \backslash E_{i}} \operatorname{dist}\left(x, E_{i}\right)=0,
$$

and since $\partial A_{k_{n_{S}}^{N_{s}}} \subset \partial \widetilde{B}_{N_{s}} \subset \partial \widehat{B}_{s}$, and

$$
\limsup _{s \rightarrow \infty} \mathcal{H}^{1}\left(\partial \widehat{B}_{s} \backslash \partial A_{k_{n_{S}}^{N_{s}}}\right) \leqq \limsup _{s \rightarrow \infty}\left(\mathcal{H}^{1}\left(\zeta_{s}\right)+\mathcal{H}^{1}\left(\partial \widetilde{B}_{N_{s}} \backslash \partial A_{k_{n_{N_{S}}}^{N_{s}}}\right)\right)=0 .
$$

Moreover, since $\partial \Omega \cap\left(\partial \widehat{E}_{i}^{s} \backslash \partial E_{i}\right) \subset\left\{0<\operatorname{dist}\left(\cdot, E_{i}\right)<t_{s}\right\}$ for any $s$ and $i$, we have

$$
\begin{aligned}
& \limsup _{s \rightarrow \infty} \mathcal{H}^{1}\left(\partial \Omega \cap\left(\partial \widehat{E}_{i}^{s} \backslash \partial E_{i}\right)\right) \\
& \quad \leqq \lim _{s \rightarrow \infty} \mathcal{H}^{1}\left(\partial \Omega \cap\left\{0<\operatorname{dist}\left(\cdot, E_{i}\right)<t_{s}\right\}\right)=0
\end{aligned}
$$

for any $i$ since $t_{s} \searrow 0$. If $D \subset \subset E_{i}$, then $D \subset \subset \widehat{E}_{i}^{s}$ provided that $s$ is large. This, and the relations $\mathbb{R}^{2} \backslash \widetilde{B}_{N_{s}}=\mathbb{R}^{2} \backslash{\widehat{B_{s}}}_{s}$ and $\operatorname{Int}\left(\widehat{B}_{s}\right) \subset \operatorname{Int}\left(\widetilde{B}_{N_{s}}\right)$ imply the local uniform convergence of $\operatorname{sdist}\left(\cdot, \partial \widehat{B}_{S}\right)$ to $\operatorname{sdist}(\cdot, \partial A)$ in $\mathbb{R}^{2}$. Thus, $\left\{\widehat{B}_{s}\right\}$ and $\left\{A_{k_{n_{N_{S}}}^{N_{s}}}\right\}$ satisfy (a)-(e).

Step 3: Construction of $\left\{B_{l}\right\}$ and $\left\{A_{k_{l}}\right\}$ satisfying $(a)-(f)$. Consider $C_{s}:=$ $\operatorname{Int}\left(\widehat{B}_{S}\right) \backslash \bigcup_{i} \widehat{E}_{i}^{s}$. Since $\left|E_{i}^{s} \Delta E_{i}\right| \rightarrow 0$ and $\left|\operatorname{Int}\left(\widehat{B}_{s}\right) \Delta \operatorname{Int}(A)\right| \rightarrow 0$ as $s \rightarrow \infty$, we have $\left|C_{S}\right| \rightarrow 0$. Therefore, applying the Area Formula with $\operatorname{dist}(\cdot, S)$, we have

$$
\left|C_{S}\right|=\int_{0}^{\infty} \mathcal{H}^{1}\left(C_{s} \cap\{\operatorname{dist}(\cdot, S)=t\}\right) d t
$$

so that, passing to further not relabelled subsequence if necessary, we can choose $t_{s}^{\prime} \in\left(0, d_{0} / 4\right)$ such that $\lim _{s \rightarrow \infty} \mathcal{H}^{1}\left(C_{s} \cap\left\{\operatorname{dist}(\cdot, S)=t_{s}^{\prime}\right\}\right)=0$, where $d_{0}$ is the minimal distance between connected components of $S$. Now the sequence

$$
B_{S}:=\widehat{B}_{S} \backslash\left(C_{S} \cap\left\{\operatorname{dist}(\cdot, S)=t_{S}^{\prime}\right\}\right)
$$

and the subsequence $\left\{A_{k_{N_{S}}^{N_{s}}}\right\}$ satisfy all assertions of the proposition. 
Proposition 3.7. Let $Q \subset \mathbb{R}^{n}$ be a connected open set and $\left\{u_{k}\right\} \subset H_{\mathrm{loc}}^{1}\left(Q ; \mathbb{R}^{n}\right)$ be such that

$$
\sup _{k} \int_{Q}\left|e\left(u_{k}\right)\right|^{2} d x<+\infty \text {. }
$$

Then either $\left|u_{k}\right| \rightarrow \infty$ a.e. in $Q$ or there exist $u \in H_{\mathrm{loc}}^{1}\left(Q ; \mathbb{R}^{n}\right) \cap G S B D^{2}\left(Q ; \mathbb{R}^{n}\right)$ and a subsequence $\left\{u_{k_{l}}\right\}$ such that $u_{k_{l}} \rightarrow u$ in $H_{\mathrm{loc}}^{1}\left(Q ; \mathbb{R}^{n}\right)$, and hence, $u_{k_{l}} \rightarrow u$ a.e. in $Q$.

Proof. Indeed, suppose that there exists a ball $B_{\epsilon} \subset \subset Q$, a measurable function $\tilde{u}: B_{\epsilon} \rightarrow \mathbb{R}^{n}$ and a not relabelled subsequence $\left\{u_{k}\right\}$ such that $u_{k} \rightarrow \widetilde{u}$ a.e. in some subset $E$ of $B_{\epsilon}$ with positive measure. Since $u_{k} \in H^{1}\left(B_{\epsilon} ; \mathbb{R}^{n}\right)$, by the PoincaréKorn inequality, there exists a rigid displacement $a_{k}: \mathbb{R}^{n} \rightarrow \mathbb{R}^{n}$ such that

$$
\left\|u_{k}+a_{k}\right\|_{H^{1}\left(B_{\epsilon}\right)}^{2} \leqq C \int_{B_{\epsilon}}\left|e\left(u_{k}\right)\right|^{2} \mathrm{~d} x
$$

for some $C>1$ independent of $k$. In particular, by the Rellich-Kondrachov Theorem, there exists $v \in H^{1}\left(B_{\epsilon} ; \mathbb{R}^{n}\right)$ such that $u_{k}+a_{k} \rightarrow v$ in $H^{1}\left(B_{\epsilon} ; \mathbb{R}^{n}\right.$ ) (up to a subsequence) and a.e. in $B_{\epsilon}$. Since $u_{k} \rightarrow \widetilde{u}$ a.e. in $E, a_{k} \rightarrow v-\widetilde{u}$ a.e. in $E$ as $k \rightarrow \infty$. Thus, $v-\tilde{u}$ is a restriction in $E$ of some rigid displacement $a: \mathbb{R}^{n} \rightarrow \mathbb{R}^{n}$. By linearity of rigid displacements, $a_{k} \rightarrow a$ pointwise in $\mathbb{R}^{n}$. Therefore, $u_{k} \rightarrow v-a$ in $H^{1}\left(B_{\epsilon} ; \mathbb{R}^{n}\right)$, hence a.e. in $B_{\epsilon}$. In view of (3.16), $\left\{u_{k}\right\} \subset G S B D^{2}\left(Q ; \mathbb{R}^{n}\right)$ with $J_{u_{k}}=\emptyset$. Hence, by [15, Theorem 1.1], there exist a further not relabelled subsequence $\left\{u_{k}\right\}$ for which the set

$$
F:=\left\{x \in Q:\left|u_{k}(x)\right| \rightarrow \infty\right\}
$$

has a finite perimeter in $\Omega$ and $u \in G S B D^{2}\left(Q ; \mathbb{R}^{n}\right)$ such that $u_{k} \rightarrow u$ a.e. in $Q \backslash F$ and

$$
\mathcal{H}^{n-1}\left(J_{u} \backslash \partial^{*} F\right)+\mathcal{H}^{n-1}\left(Q \cap \partial^{*} F\right) \leqq \liminf _{k \rightarrow \infty} \mathcal{H}^{1}\left(J_{u_{k}}\right)=0
$$

Thus, $P(F, Q)=0$, i.e., either $F=\emptyset$ or $F=Q$. Since $u_{k} \rightarrow u=v-a$ a.e. in $B_{\epsilon} \subset Q$, the case $F=Q$ is not possible. Thus, $F=\emptyset$. By (3.17), $\mathcal{H}^{1}\left(J_{u}\right)=0$.

Now we show that $u_{k} \rightarrow u$ in $H_{\mathrm{loc}}^{1}\left(Q ; \mathbb{R}^{n}\right)$ and $u \in H_{\mathrm{loc}}^{1}\left(Q ; \mathbb{R}^{n}\right)$. Let $D_{1} \subset \subset$ $D_{2} \subset \subset \ldots$ be an increasing sequence of connected Lipschitz open sets such that $D_{1}:=B_{\epsilon}$ and $Q=\cup_{j} D_{j}$. Applying Poincaré-Korn inequality $D_{j}$ we find a rigid displacement $a_{k}^{j}$ such that

$$
\left\|u_{k}+a_{k}^{j}\right\|_{H^{1}\left(D_{j}\right)}^{2} \leqq c_{j} \int_{D_{j}}\left|e\left(u_{k}\right)\right|^{2} \mathrm{~d} x,
$$

where $c_{j}$ is independent on $k$. Then by the Rellich-Kondrachov Theorem, every subsequence $\left\{u_{k_{l}}\right\}$ admits further not relabelled subsequence such that $u_{k_{l}}+a_{k_{l}}^{j} \rightarrow v$ in $H^{1}\left(D_{j} ; \mathbb{R}^{n}\right)$ and a.e. in $D_{j}$ for some $v \in H^{1}\left(D_{j} ; \mathbb{R}^{n}\right)$. Since $u_{k_{l}} \rightarrow u$ a.e. in $D_{j}$, it follows that $a_{k_{l}}^{j} \rightarrow v-u$ a.e. in $D_{j}$ and hence, $v-u$ is also a rigid displacement. 
Since a.e.-convergence of linear functions implies the local strong $H^{1}$-convergence, $u_{k_{l}} \rightarrow u$ in $H^{1}\left(D_{j} ; \mathbb{R}^{n}\right)$, and thus, $u \in H^{1}\left(D_{j} ; \mathbb{R}^{n}\right)$. Since the subsequence $\left\{u_{k_{l}}\right\}$ is arbitrary, $u_{k} \rightarrow u$ in $H^{1}\left(D_{j} ; \mathbb{R}^{n}\right)$. By the choice of $D_{j}, u_{k} \rightarrow u$ in $H_{\text {loc }}^{1}\left(Q ; \mathbb{R}^{n}\right)$ and $u \in H_{\text {loc }}^{1}\left(Q ; \mathbb{R}^{n}\right)$.

The following corollary of Proposition 3.7 is used in the proof of Theorem 2.7:

Corollary 3.8. Let $P, P_{k} \subset \mathbb{R}^{n}$ be connected bounded open sets such that for any $G \subset \subset P$ there exists $k_{G}$ such that $G \subset \subset P_{k}$ for all $k>k_{G}$, and let $u_{k} \in$ $H_{\mathrm{loc}}^{1}\left(P_{k} ; \mathbb{R}^{n}\right)$ be such that

$$
\sup _{k} \int_{P_{k}}\left|e\left(u_{k}\right)\right|^{2} d x<\infty .
$$

Then there exist $u \in H_{\mathrm{loc}}^{1}\left(P ; \mathbb{R}^{n}\right) \cap G S B D^{2}\left(P ; \mathbb{R}^{n}\right)$, a subsequence $\left\{\left(P_{k_{l}}, u_{k_{l}}\right)\right\}$ and a sequence $\left\{b_{l}^{P}\right\}$ of rigid displacements such that $u_{k_{l}}+b_{l}^{P} \rightarrow$ u a.e. in $P$.

Proof. Let $B_{\epsilon} \subset \subset P$ be any ball. By assumption, $B_{\epsilon} \subset \subset P_{k}$ for all large $k$. By the Poincaré-Korn inequality, for all such $k$ there exists a rigid displacement $b_{k}^{\epsilon}$ such that

$$
\left\|u_{k}+b_{k}^{\epsilon}\right\|_{H^{1}\left(B_{\epsilon}\right)}^{2} \leqq C_{\epsilon} \int_{B_{\epsilon}}\left|e\left(u_{k}\right)\right|^{2} \mathrm{~d} x .
$$

This, (3.18) and the Rellich-Kondrachov Theorem imply that there exist a not relabelled subsequence $\left\{u_{k}+b_{k}^{\epsilon}\right\}$ and $v \in H^{1}\left(B_{\epsilon} ; \mathbb{R}^{n}\right)$ such that $u_{k}+b_{k}^{\epsilon} \rightarrow v$ as $k \rightarrow \infty$ in $H^{1}\left(B_{\epsilon} ; \mathbb{R}^{n}\right)$, hence, a.e. in $B_{\epsilon}$. Now applying Proposition 3.7 with an increasing sequence $\left\{G_{i}\right\}$ of connected open sets satisfying $G_{1}=B_{\epsilon}, G_{i} \subset \subset P$ and $P=\cup_{i} G_{i}$ we find $u \in H_{\mathrm{loc}}^{1}\left(P ; \mathbb{R}^{n}\right) \cap G S B D^{2}\left(P ; \mathbb{R}^{n}\right)$ with $u=v$ in $B_{\epsilon}$ and a not relabelled subsequence $\left\{u_{k}+b_{k}^{\epsilon}\right\}$ such that $u_{k}+b_{k}^{\epsilon} \rightarrow u$ as $k \rightarrow \infty$ a.e. in $P$.

Proposition 3.9. Assume (H1)-(H2) and let $x_{0} \in \Sigma, \delta \in\left(0, \frac{1}{2}\right)$ and $r \in(0,1)$ be such that $v_{0}:=v_{\Sigma}\left(x_{0}\right)$ exists. Then

$$
\left|\varphi(y, \xi)-\varphi\left(x_{0}, \xi\right)\right|<\delta
$$

for any $y \in U_{r, v_{0}}\left(x_{0}\right)$ and $\xi \in \mathbb{S}^{1}, U_{r, v_{0}}\left(x_{0}\right) \cap \Sigma$ is a graph of a Lipschitz function over tangent line $U_{r, v_{0}}\left(x_{0}\right) \cap T_{x_{0}}$ in direction $v_{0}$ and

$$
\int_{U_{r, v_{0}}\left(x_{0}\right) \cap \Sigma}\left|\beta(y)-\beta\left(x_{0}\right)\right| d \mathcal{H}^{1}<\delta \mathcal{H}^{1}\left(U_{r, v_{0}}\left(x_{0}\right) \cap \Sigma\right) .
$$

Let $A \in \mathcal{A}_{m}$ be such that $x_{0} \in \Sigma \cap \partial^{*} A, U_{r, v_{0}}\left(x_{0}\right) \cap\left\{\operatorname{dist}\left(\cdot, T_{x_{0}}\right) \geqq \delta r\right\} \subset \operatorname{Int}(A) \cup S$, and let $\left\{\left(A_{k}, u_{k}\right)\right\} \subset \mathcal{C}_{m}$ and $u \in H_{\mathrm{loc}}^{1}\left(\operatorname{Int}(A) ; \mathbb{R}^{2}\right)$ be such that $A_{k} \stackrel{\tau_{\mathcal{A}}}{\rightarrow} A$ and

$$
\sup _{k} \int_{U_{r, v_{0}}\left(x_{0}\right) \cap\left(A_{k} \cup S\right)}\left|e\left(u_{k}\right)\right|^{2} d x+\mathcal{H}^{1}\left(U_{r, \nu_{0}}\left(x_{0}\right) \cap \partial A_{k}\right)<\infty
$$


and $u_{k} \rightarrow$ u a.e. in $U_{r, v_{0}}\left(x_{0}\right) \cap \operatorname{Int}(A)$ and $\left|u_{k}\right| \rightarrow+\infty$ a.e. in $S \cap U_{r, v_{0}}\left(x_{0}\right)$. Then there exists $k_{\delta}>1$ for which

$$
\begin{aligned}
& \int_{U_{r, v_{0}}\left(x_{0}\right) \cap \Omega \cap \partial^{*} A_{k}} \varphi\left(x, v_{A_{k}}\right) d \mathcal{H}^{1}+2 \int_{U_{r, v_{0}}\left(x_{0}\right) \cap \Omega \cap\left(A_{k}^{(0)} \cup A_{k}^{(1)}\right) \cap \partial A_{k}} \varphi\left(x, v_{A_{k}}\right) d \mathcal{H}^{1} \\
& \quad+\int_{U_{r, v_{0}}\left(x_{0}\right) \cap \Sigma \cap A_{k}^{(0)} \cap \partial A_{k}}\left(\varphi\left(x, v_{\Sigma}\right)+\beta\right) d \mathcal{H}^{1} \\
& \quad+\int_{U_{r, v_{0}}\left(x_{0}\right) \cap \Sigma \cap \partial^{*} A_{k} \backslash J_{u_{k}}} \beta d \mathcal{H}^{1}+\int_{U_{r, v_{0}}\left(x_{0}\right) \cap J_{u_{k}}} \varphi\left(x, v_{\Sigma}\right) d \mathcal{H}^{1} \\
& \geqq \frac{1}{1+\frac{\delta}{c_{2}}} \int_{U_{r, v_{0}}\left(x_{0}\right) \cap \Sigma \cap \partial^{*} A} \varphi\left(x, v_{\Sigma}\right) d \mathcal{H}^{1}-\delta \int_{U_{r, v_{0}}\left(x_{0}\right) \cap \Sigma} \varphi\left(x, v_{\Sigma}\right) d \mathcal{H}^{1} .
\end{aligned}
$$

for any $k>k_{\delta}$.

We postpone the proof unfil after the following lemma:

Lemma 3.10. Let $\phi$ be a norm in $\mathbb{R}^{2}, A \in \mathcal{A}_{m}$ be such that $0 \in \Sigma \cap \partial^{*} A$, $U_{r} \cap\left\{\operatorname{dist}\left(\cdot,\left\{x_{2}=0\right\}\right) \geqq \frac{r}{2}\right\} \subset \operatorname{Int}(A) \cup S$, and $\left\{\left(A_{k}, u_{k}\right)\right\} \subset \mathcal{C}_{m}$, and $u \in$ $H_{\mathrm{loc}}^{1}\left(\operatorname{Int}(A) ; \mathbb{R}^{2}\right)$ be such that

$$
\sup _{k} \int_{U_{r} \cap A_{k}}\left|e\left(u_{k}\right)\right|^{2} d x+\mathcal{H}^{1}\left(U_{r} \cap \partial A_{k}\right)<\infty
$$

and $A_{k} \stackrel{\tau_{\mathcal{A}}}{\rightarrow} A$ and $u_{k} \rightarrow u$ a.e. in $U_{r} \cap \operatorname{Int}(A)$ and $\left|u_{k}\right| \rightarrow+\infty$ a.e. in $S \cap U_{r}$. Then for every $\epsilon>0$ there exists $k_{\epsilon}>0$ such that for any $k>k_{\epsilon}$,

$$
\begin{aligned}
& \int_{U_{r} \cap \Omega \cap \partial^{*} A_{k}} \phi\left(v_{A_{k}}\right) d \mathcal{H}^{1}+2 \int_{U_{r} \cap \Omega \cap A_{k}^{(1)} \cap \partial A_{k}} \phi\left(v_{A_{k}}\right) d \mathcal{H}^{1} \\
& \quad \geqq 2 \int_{U_{r} \cap \Sigma \cap\left(\partial^{*} A_{k} \backslash J_{u_{k}}\right)} \phi\left(v_{\Sigma}\right) d \mathcal{H}^{1}-\epsilon .
\end{aligned}
$$

Proof. Since $\left(A_{k}^{(1)} \cap \partial A_{k}\right) \cup J_{u_{k}}$ is $\mathcal{H}^{1}$-rectifiable, by [3, pp. 80] there exists at most countably many $C^{1}$-curves $\left\{\Gamma_{i}^{k}\right\}_{i \geqq 1}$ such that

$$
\mathcal{H}^{1}\left(\left(\left(A_{k}^{(1)} \cap \partial A_{k}\right) \cup J_{u_{k}}\right) \backslash \bigcup_{i \geqq 1} \Gamma_{i}^{k}\right)=0 .
$$

Selecting closed arcs inside curves if necessary, we suppose that $\Gamma_{i}^{k} \subset U_{r}$ and

$$
\int_{U_{r} \cap A_{k}^{(1)} \cap \partial A_{k}} \phi\left(v_{A_{k}}\right) \mathrm{d} \mathcal{H}^{1}+\int_{U_{r} \cap J_{u_{k}}} \phi\left(v_{A_{k}}\right) \mathrm{d} \mathcal{H}^{1}+\epsilon>\sum_{i \geqq 1} \int_{\Gamma_{i}^{k}} \phi\left(v_{\Gamma_{i}^{k}}\right) \mathrm{d} \mathcal{H}^{1}
$$

for any $k$. Since each $\Gamma_{i}^{k}$ is $C^{1}$, we can choose a Lipschitz open set $V_{i}^{k} \subset U_{r}$ such that $\Gamma_{i}^{k} \subset \overline{V_{i}^{k}},\left|V_{i}^{k}\right| \leqq 2^{-i-1-k}$,

$$
\int_{\partial V_{i}^{k}} \phi\left(v_{V_{i}^{k}}\right) \mathrm{d} \mathcal{H}^{1}<2 \int_{\Gamma_{i}^{k}} \phi\left(v_{\Gamma_{i}^{k}}\right) \mathrm{d} \mathcal{H}^{1}+\frac{\epsilon}{2^{i+1}}
$$


and $\operatorname{dist}_{\mathcal{H}}\left(\Gamma_{i}^{k}, \partial V_{i}^{k}\right)<2^{-k}$, where $\operatorname{dist}_{\mathcal{H}}$ is the Hausdorff distance (see e.g., (A.1) for the definition). Let $V_{0}^{k}:=U_{r} \backslash \overline{\operatorname{Int}\left(A_{k}\right) \cup S}$ be the "voids". By the definition of $\left\{V_{i}^{k}\right\}$,

$$
\begin{aligned}
& \int_{U_{r} \cap \Omega \cap \partial^{*} A_{k}} \phi\left(v_{A_{k}}\right) \mathrm{d} \mathcal{H}^{1}+\int_{U_{r} \cap\left(\Sigma \backslash \partial^{*} A_{k}\right)} \phi\left(v_{\Sigma}\right) \mathrm{d} \mathcal{H}^{1}+2 \int_{U_{r} \cap J_{u_{k}}} \phi\left(v_{A_{k}}\right) \mathrm{d} \mathcal{H}^{1} \\
& +2 \int_{U_{r} \cap \Omega \cap A_{k}^{(1)} \cap \partial A_{k}} \phi\left(v_{A_{k}}\right) \mathrm{d} \mathcal{H}^{1} \geqq \sum_{i \geqq 0} \int_{\partial V_{i}^{k}} \phi\left(v_{\partial V_{i}^{k}}\right) \mathrm{d} \mathcal{H}^{1}-\frac{\epsilon}{2}
\end{aligned}
$$

In particular, by (3.22), sup $\sum_{i \geqq 0} \mathcal{H}^{1}\left(\partial V_{i}^{k}\right)<\infty$, and hence, by [46, Proposition 2.6], there exists $\xi \in \mathbb{R}^{2}$ such that the set $\left\{x \in \bigcup_{i} \partial V_{i}: \operatorname{tr}_{U_{r} \backslash \cup V_{i}^{k}}(u)(x)=\xi\right\}$ is $\mathcal{H}^{1}$ negligible. Define

$$
w_{k}:=u_{k} \chi_{U_{r} \backslash \bigcup_{i} V_{i}^{k}}+\xi \chi_{\bigcup_{i} V_{i}^{k}}
$$

Then $w_{k} \in G S B D^{2}\left(U_{r} ; \mathbb{R}^{2}\right), J_{w_{k}}=\bigcup_{i} \partial V_{i}^{k}$ and by (3.22),

$$
\sup _{k} \int_{U_{r}}\left|e\left(w_{k}\right)\right|^{2} \mathrm{~d} x+\mathcal{H}^{1}\left(J_{w_{k}}\right)<\infty .
$$

Since $\sum_{i \geqq 1}\left|V_{i}^{k}\right| \leqq 2^{-k}$, by assumption on $\left\{u_{k}\right\}$ and $\left\{A_{k}\right\}$,

$$
w_{k} \rightarrow \begin{cases}u & \text { a.e. in } U_{r} \cap \operatorname{Int}(A), \\ \xi & \text { a.e. in } \Omega \cap U_{r} \cap \backslash \bar{A}\end{cases}
$$

and $\left|w_{k}\right| \rightarrow+\infty$ a.e. in $U_{r} \cap S$.

We show that

$$
2 \int_{U_{r} \cap \Sigma} \phi\left(v_{\Sigma}\right) \mathrm{d} \mathcal{H}^{1} \leqq \liminf _{k \rightarrow \infty} \int_{J_{w_{1}}} \phi\left(v_{J_{w_{1}}}\right) \mathrm{d} \mathcal{H}^{1} .
$$

By assumption, $U_{r} \cap \Sigma \subset(-1,1) \times(-\epsilon, \epsilon)$ and $U_{r} \cap \partial A \subset(-1,1) \times(-\epsilon, \epsilon)$, thus, by the convergence $A_{k} \stackrel{\tau_{\mathcal{A}}}{\rightarrow} A$ and Remark 2.2, $U_{r} \cap \partial A_{k} \subset(-1,1) \times(-\epsilon, \epsilon)$ for all large $k$. In particular, for such $k, J_{w_{k}} \subset(-1,1) \times(-\epsilon, \epsilon)$.

Under the notation of [15], given $\xi \in \mathbb{S}^{1}$ let $\pi_{\xi}$ be the orthogonal projection onto the line $\Pi_{\xi}:=\left\{\eta \in \mathbb{R}^{2}: \xi \cdot \eta=0\right\}$, perpendicular to $\xi$; given a Borel set $F \subset \mathbb{R}^{2}$ and $y \in \Pi_{\xi}$, let $F_{y}^{\xi}:=\{t \in \mathbb{R}: y+t \xi \in F\}$ be the one-dimensional slice of $F$, and given $u \in G S B D\left(U_{r} ; \mathbb{R}^{2}\right)$ and $y \in \Pi_{\xi}$, let $\widehat{u}_{y}^{\xi}(t)=u(y+t \xi) \cdot \xi$ be the one-dimensional slice of $u$. Since $w_{h} \rightarrow w$ a.e. in $U_{r} \backslash S$, by [15, Eq. 3.23], for any $\epsilon>0$ and Borel set $F \subset U_{r}$,

$$
\mathcal{H}^{0}\left((F \backslash S)_{y}^{\xi} \cap J_{\widehat{w}_{y}^{\xi}}\right)+\mathcal{H}^{0}\left(\left(U_{r} \cap \Sigma\right)_{y}^{\xi}\right) \leqq \liminf _{k \rightarrow \infty}\left(\mathcal{H}^{0}\left(F_{y}^{\xi} \cap J_{\left(\widehat{w}_{k}\right)_{y}^{\xi}}\right)+\epsilon f_{y}^{\xi}\left(w_{k}\right)\right)
$$


for a.e. $\xi \in \mathbb{S}^{1}$ and a.e. $y \in \Pi_{\xi}$, where the integral of $f_{y}^{\xi}\left(w_{k}\right)$ over $\Pi_{\xi}$ is uniformly bounded independent on $\xi$ and $k$ (see also (4.21) below).

Let

$$
\widehat{A}:=\left\{y \in \pi_{\xi}\left(U_{r} \cap \Sigma\right): \liminf _{k \rightarrow \infty} \mathcal{H}^{0}\left(J_{\left(\widehat{w}_{k}\right)_{y}^{\xi}}\right)=0\right\} \subset \Pi_{\xi} .
$$

Then $F:=U_{r} \cap \pi_{\xi}^{-1}(\widehat{A})$ is Borel and, thus, integrating (3.26) over $\widehat{A}$ and using the definition of $\widehat{A}$ and Fatou's Lemma we get

$$
\mathcal{H}^{1}\left(\pi_{\xi}\left(U_{r} \cap \Sigma\right) \cap \widehat{A}\right) \leqq \liminf _{k \rightarrow \infty} \epsilon \int_{\widehat{A}} f_{y}^{\xi}\left(v_{k}\right) \mathrm{d} y \leqq M \epsilon
$$

for some $M>0$ independent of $\epsilon$. Thus, letting $\epsilon \rightarrow 0$ we get $\mathcal{H}^{1}(\widehat{A})=0$. In particular,

$$
\limsup _{k \rightarrow \infty} \mathcal{H}^{1}\left(\pi_{\xi}\left(U_{r} \cap \Sigma\right) \backslash \pi_{\xi}\left(J_{w_{k}}\right)\right)=0 .
$$

Note that by construction, $J_{w_{k}}$ is a union of open sets, thus, for a.e. $y \in \pi_{\xi}\left(J_{w_{k}}\right)$, the line $\pi_{\xi}^{-1}(y)$ passing through $y$ and parallel to $\xi$ crosses $J_{w_{k}}$ at least at two points. Thus,

$$
\mathcal{H}^{0}\left(J_{\left(\widehat{w}_{k}\right)_{y}^{\xi}}\right) \geqq 2=2 \mathcal{H}^{0}\left(\left(U_{r} \cap \Sigma\right)_{y}^{\xi}\right)
$$

for $\mathcal{H}^{1}$-a.e. $y \in \pi_{\xi}\left(J_{w_{k}}\right) \cap \pi_{\xi}\left(U_{r} \cap \Sigma\right)$, where $o(1) \rightarrow 0$ as $k \rightarrow \infty$. Now we choose arbitrary pairwise disjoint open sets $F_{1}, F_{2}, \ldots \subset \subset U_{r}$ and repeating the same argument of Step 1 in the proof of Proposition 4.6 (by using (3.28) in place of (4.20) and using (3.27)) we obtain (3.25).

From (3.25) and (3.24) it follows that there exists $k_{\epsilon}>0$ such that

$$
\begin{aligned}
& \int_{U_{r} \cap \Omega \cap \partial^{*} A_{k}} \phi\left(v_{A_{k}}\right) \mathrm{d} \mathcal{H}^{1}+\int_{U_{r} \cap\left(\Sigma \backslash \partial^{*} A_{k}\right)} \phi\left(v_{\Sigma}\right) \mathrm{d} \mathcal{H}^{1}+2 \int_{U_{r} \cap J_{u_{k}}} \phi\left(v_{A_{k}}\right) \mathrm{d} \mathcal{H}^{1} \\
& +2 \int_{U_{r} \cap \Omega \cap A_{k}^{(1)} \cap \partial A_{k}} \phi\left(v_{A_{k}}\right) \mathrm{d} \mathcal{H}^{1} \geqq 2 \int_{U_{r} \cap \Sigma} \phi\left(v_{\Sigma}\right) \mathrm{d} \mathcal{H}^{1}-\epsilon
\end{aligned}
$$

for any $k>k_{\epsilon}$. Now (3.23) follows from (3.29).

We anticipate here that in Lemma 4.7, below, we establish a similar result.

Proof of Proposition 3.9. For simplicity, assume that $x_{0}=0, v=\mathbf{e}_{2}$ and $\phi(\xi)=$ $\varphi(0, \xi)$. Denote the left-hand side of (3.21) by $\alpha_{k}$. By (3.19) and (2.5),

$$
\alpha_{k} \geqq \widehat{\alpha}_{k}-2 \delta \mathcal{H}^{1}\left(U_{r} \cap \Omega \cap \partial A_{k}\right),
$$

where

$$
\begin{aligned}
\widehat{\alpha}_{k}:= & \int_{U_{r} \cap \Omega \cap \partial^{*} A_{k}} \phi\left(v_{A_{k}}\right) \mathrm{d} \mathcal{H}^{1}+2 \int_{U_{r} \cap \Omega \cap\left(A_{k}^{(0)} \cup A_{k}^{(1)}\right) \cap \partial A_{k}} \phi\left(v_{A_{k}}\right) \mathrm{d} \mathcal{H}^{1} \\
& +\int_{U_{r} \cap \Sigma \cap \partial^{*} A_{k} \backslash J_{u_{k}}} \beta \mathrm{d} \mathcal{H}^{1}+\int_{U_{r} \cap J_{u_{k}}} \varphi\left(x, v_{\Sigma}\right) \mathrm{d} \mathcal{H}^{1} .
\end{aligned}
$$


By Lemma 3.10 applied with $\phi$ and $\epsilon:=\delta \int_{U_{r} \cap \Sigma} \varphi\left(x, v_{\Sigma}\right) \mathrm{d} \mathcal{H}^{1}$, there exists $k_{\delta}$ such that

$$
\widehat{\alpha}_{k} \geqq 2 \int_{U_{r} \cap \Sigma \cap\left(\partial^{*} A_{k} \backslash J_{u_{k}}\right)} \phi\left(v_{\Sigma}\right) \mathrm{d} \mathcal{H}^{1}+\int_{U_{r} \cap \Sigma \cap \partial^{*} A_{k} \backslash J_{u_{k}}} \beta \mathrm{d} \mathcal{H}^{1}+\int_{U_{r} \cap J_{u_{k}}} \phi\left(v_{\Sigma}\right) \mathrm{d} \mathcal{H}^{1}-\epsilon
$$

for all $k>k_{\delta}$. Then by (3.19)

$$
\begin{aligned}
\int_{U_{r} \cap \Sigma \cap\left(\partial^{*} A_{k} \backslash J_{u_{k}}\right)} \phi\left(v_{\Sigma}\right) \mathrm{d} \mathcal{H}^{1} \geqq & \int_{U_{r} \cap \Sigma \cap\left(\partial^{*} A_{k} \backslash J_{u_{k}}\right)} \varphi\left(x, v_{\Sigma}\right) \mathrm{d} \mathcal{H}^{1} \\
& -\delta \mathcal{H}^{1}\left(U_{r} \cap \Sigma \cap\left(\partial^{*} A_{k} \backslash J_{u_{k}}\right)\right),
\end{aligned}
$$

and therefore,

$$
\begin{aligned}
\widehat{\alpha}_{k} \geqq & \int_{U_{r} \cap \Sigma \cap\left(\partial^{*} A_{k} \backslash J_{u_{k}}\right)}\left(2 \varphi\left(x, v_{\Sigma}\right)+\beta\right) \mathrm{d} \mathcal{H}^{1}+\int_{U_{r} \cap J_{u_{k}}} \phi\left(v_{\Sigma}\right) \mathrm{d} \mathcal{H}^{1} \\
& -\epsilon-\delta \mathcal{H}^{1}\left(U_{r} \cap \Sigma \cap\left(\partial^{*} A_{k} \backslash J_{u_{k}}\right)\right)
\end{aligned}
$$

Applying (2.5) in the first integral we get

$$
\widehat{\alpha}_{k} \geqq \int_{U_{r} \cap \Sigma \cap \partial^{*} A_{k}} \varphi\left(x, v_{\Sigma}\right) \mathrm{d} \mathcal{H}^{1}-\epsilon-\delta \mathcal{H}^{1}\left(U_{r} \cap \Sigma \cap \partial^{*} A_{k}\right)
$$

so that

$$
\alpha_{k} \geqq \int_{U_{r} \cap \Sigma \cap \partial^{*} A_{k}} \varphi\left(x, \nu_{\Sigma}\right) \mathrm{d} \mathcal{H}^{1}-\epsilon-2 \delta \mathcal{H}^{1}\left(U_{r} \cap \partial A_{k}\right) .
$$

By (2.4) and (2.5)

$$
c_{1} \mathcal{H}^{1}\left(U_{r} \cap \partial A_{k}\right) \leqq \alpha_{k}+\int_{U_{1} \cap \Sigma} \varphi\left(x, v_{\Sigma}\right) \mathrm{d} \mathcal{H}^{1},
$$

and hence, (3.31) and the definition of $\epsilon$ imply

$$
\left(1+\frac{\delta}{c_{1}}\right) \alpha_{k} \geqq \int_{U_{r} \cap \Sigma \cap \partial^{*} A_{k}} \varphi\left(x, v_{\Sigma}\right) \mathrm{d} \mathcal{H}^{1}-\delta\left(1+\frac{\delta}{c_{1}}\right) \int_{U_{r} \cap \Sigma} \varphi\left(x, v_{\Sigma}\right) \mathrm{d} \mathcal{H}^{1} .
$$

and (3.21) follows.

Finally we prove compactness of $\mathcal{C}_{m}$.

Proof of Theorem 2.7. Let $R:=\sup _{k} \mathcal{F}\left(A_{k}, u_{k}\right)$ and, by passing to a further not relabelled subsequence if necessary, we assume that

$$
\liminf _{k \rightarrow \infty} \mathcal{F}\left(A_{k}, u_{k}\right)=\lim _{k \rightarrow \infty} \mathcal{F}\left(A_{k}, u_{k}\right) .
$$

By (H1)-(H3) we have

$$
\sup _{k}\left(c_{1} \mathcal{H}^{1}\left(\Omega \cap \partial A_{k}\right)+2 c_{3} \int_{A_{k} \cup S}\left|e\left(u_{k}\right)\right|^{2} \mathrm{~d} x\right) \leqq R+\int_{\Sigma}|\beta| \mathrm{d} \mathcal{H}^{1}
$$


and hence,

$$
\mathcal{H}^{1}\left(\partial A_{k}\right) \leqq \mathcal{H}^{1}\left(\Omega \cap \partial A_{k}\right)+\mathcal{H}^{1}\left(\partial \Omega \cap \partial A_{k}\right) \leqq \frac{R+\int_{\Sigma}|\beta| \mathrm{d} \mathcal{H}^{1}}{c_{1}}+\mathcal{H}^{1}(\partial \Omega)
$$

and

$$
\int_{A_{k} \cup S}\left|e\left(u_{k}\right)\right|^{2} \mathrm{~d} x \leqq \frac{R+\int_{\Sigma}|\beta| \mathrm{d} \mathcal{H}^{1}}{2 c_{3}}
$$

for any $k \geqq 1$. In view of (3.32) and Proposition 3.3, there exists $A \in \mathcal{A}_{m}$ with $\mathcal{H}^{1}(\partial A)<\infty$ and a not relabelled subsequence $\left\{A_{k}\right\}$ such that $\operatorname{sdist}\left(\cdot, \partial A_{k}\right) \rightarrow$ $\operatorname{sdist}(\cdot, \partial A)$ locally uniformly in $\mathbb{R}^{2}$. Now we construct the sequence $\left\{\left(B_{n}, v_{n}\right)\right\}$ in three steps. In the first step we apply Proposition 3.6 and Corollary 3.8 to obtain a (not relabelled) subsequence and to construct a sequence $\left\{B_{k}\right\} \subset \mathcal{A}_{m}$ with associated piecewise rigid displacements $\left\{a_{k}\right\}$ such that both $B_{k} \stackrel{{ }^{\tau} \mathcal{A}}{\rightarrow} A$ and $u_{k}+a_{k} \rightarrow u$ a.e. in $\operatorname{Int}(A) \cup S$ for some $u \in H_{\text {loc }}^{1}\left(\operatorname{Int}(A) \cup S, \mathbb{R}^{2}\right) \cap G S B D^{2}\left(\operatorname{Int}(A \cup S \cup \Sigma) ; \mathbb{R}^{2}\right)$. In the second step we take care of the fact that adding different rigid motions in $B_{k}$ and in $S$ can create extra jump at $\Sigma$ making difficult to satisfy (2.9). More precisely, by Proposition 3.9 we modify $\left\{B_{k}\right\}$ and $\left\{u_{k}\right\}$ so that the modified sequence $\left\{\left(B_{k}^{\delta}, u_{k}^{\delta}\right)\right\} \subset \mathcal{C}_{m}$ satisfies (2.9) with some small error of order $\delta>0$. Finally, in Step 3 we construct the sequence $\left\{\left(D_{n}, v_{n}\right)\right\} \subset \mathcal{C}_{m}$ by means of $\left\{\left(B_{k}^{\delta}, u_{k}^{\delta}\right)\right\}$ and a diagonal argument.

Step 1: Defining a first modification $\left\{B_{k}\right\}$ of $\left\{A_{k}\right\}$. By Proposition 3.6 there exist a not relabelled subsequence $\left\{A_{k}\right\}$ and a sequence $\left\{B_{k}\right\} \subset \mathcal{A}_{m}$ such that

(a1) $\partial A_{k} \subset \partial B_{k}$ and $\lim _{k \rightarrow \infty} \mathcal{H}^{1}\left(\partial B_{k} \backslash \partial A_{k}\right)=0$;

(a2) $B_{k} \stackrel{\tau_{\mathcal{A}}}{\rightarrow} A$ as $k \rightarrow \infty$

(a3) if $\left\{E_{i}\right\}_{i \in I}$ is all connected components of $\operatorname{Int}(A)$, there exists connected components of $\operatorname{Int}\left(B_{k}\right)$ enumerated as $\left\{E_{i}^{k}\right\}_{i \in I}$ such that for any $i$ and $G \subset \subset E_{i}$ one has $G \subset \subset E_{i}^{k}$ for all large $k$ (depending only on $i$ and $G$ );

(a4) $\sum_{i} \mathcal{H}^{1}\left(\partial \Omega \cap\left(\partial E_{i}^{k} \backslash \partial E_{i}\right)\right) \rightarrow 0$ as $k \rightarrow \infty$;

(a5) $\left|B_{k}\right|=\left|A_{k}\right|$ for all $k \geqq 1$;

(a6) the boundary of every connected component of $\operatorname{Int}\left(B_{k}\right) \backslash \bigcup_{i} E_{i}^{k}$ intersects the boundary of at most one connected component of $S$.

Notice that by condition (a1),

$$
\lim _{k \rightarrow \infty}\left|\mathcal{S}\left(A_{k}, u_{k}\right)-\mathcal{S}\left(B_{k}, u_{k}\right)\right|=0
$$

and

$$
\mathcal{W}\left(A_{k}, u_{k}\right)=\mathcal{W}\left(B_{k}, u_{k}\right)
$$

Thus,

$$
\lim _{k \rightarrow \infty}\left|\mathcal{F}\left(A_{k}, u_{k}\right)-\mathcal{F}\left(B_{k}, u_{k}\right)\right|=0 .
$$


Now we define the piecewise rigid displacements $a_{k}$ associated to $B_{k}$. Let $\left\{S_{j}\right\}_{j \in Y}$ be the set of connected components of $S$ for some index set $Y$. We define the index sets $I_{n} \subset I$ and $Y_{n} \subset Y$ inductively on $n$ in such a way that Corollary 3.8 holds with $P_{k}=E_{i}^{k}$ and $P=E_{i}$ and also with $P_{k}=P=S_{j}$ for every $i \in I_{n}$ and $j \in Y_{n}$ with the same rigid displacements $a_{k}^{n}$ independent of $i$ and $j$.

More precisely, let $I_{0}:=Y_{0}:=\varnothing$, and given the sets $I_{1}, \ldots, I_{n-1}$ and $Y_{1}, \ldots, Y_{n-1}$ for $n \geqq 1$, we define $I_{n}$ and $Y_{n}$ as follows. By Corollary 3.8 applied with $P_{k}=P=S_{j_{n}}$ with $j_{n}$ the smallest element of $Y \backslash \bigcup_{l=1}^{n-1} Y_{l}$, we find a not relabelled subsequence $\left\{\left(B_{k}, u_{k}\right)\right\}$, a sequence $\left\{a_{k}^{n}\right\}$ of rigid displacements and $w_{n} \in H_{\mathrm{loc}}^{1}\left(S_{j_{n}} ; \mathbb{R}^{2}\right)$ such that $u_{k}+a_{k}^{n} \rightarrow w_{n}$ a.e. in $S_{j_{n}}$. Let $I_{n}$ and $Y_{n}$ be the sets such that there exists a not relabelled subsequence $\left\{\left(B_{k}, u_{k}\right)\right\}$ such that the sequence $\left(u_{k}+a_{k}^{n}\right) \chi_{E_{i}^{k}}$ converges a.e. in $E_{i}$ for $i \in I_{n}$ and the sequence $\left(u_{k}+a_{k}^{n}\right) \chi_{S_{j}}$ converges a.e. in $S_{j}$ for $j \in Y_{n}$. Recall that $j_{n} \in Y_{n}$. Let

$$
F_{n}^{k}:=\left(\bigcup_{i \in I_{n}} E_{i}^{k}\right) \cup\left(\bigcup_{j \in Y_{n}} S_{j}\right) \quad \text { and } \quad F_{n}:=\left(\bigcup_{i \in I_{n}} E_{i}\right) \cup\left(\bigcup_{j \in Y_{n}} S_{j}\right) .
$$

By the definition of $I_{n}$ and $Y_{n}$, and by diagonalization the sequence $\left(u_{k}+a_{k}^{n}\right) \chi_{F_{n}^{k}}$ converges as $k \rightarrow \infty$ a.e. in $F_{n}$ to some function in $H_{\text {loc }}^{1}\left(F_{n} ; \mathbb{R}^{2}\right)$, which we still denote by $w_{n}$.

Note that for large $n, Y_{n}$ is empty since $Y$ is finite by assumption. Notice also that by definition of $I_{n}$ and $Y_{n}$, and Proposition 3.7 applied in connected open sets $P \subset \subset E_{i} \cup S_{j}$, we have $\left|u_{k}+a_{k}^{n}\right| \rightarrow+\infty$ a.e in $E_{i} \cup S_{j}$ for every $i \in I \backslash I_{n}$ and $j \in Y \backslash Y_{n}$,

We now define the rigid displacements in $E_{i}^{k}$ for $i \in I \backslash \bigcup_{n} I_{n}$. By a diagonal argument and by Corollary 3.8 applied with $P_{k}=E_{i}^{k}$ and $P=E_{i}$ for any $i \in$ $I \backslash \bigcup_{n} I_{n}$, we find a further not relabelled sequence $\left\{B_{k}, u_{k}\right\}$, sequence $\left\{\widetilde{a}_{k}^{i}\right\}$ of rigid displacements and $w^{i} \in H_{\mathrm{loc}}^{1}\left(E_{i} ; \mathbb{R}^{2}\right)$ such that $\left(u_{k}+\widetilde{a}_{k}^{i}\right) \chi_{E_{i}^{k}} \rightarrow w^{i}$ a.e. $E_{i}$ as $k \rightarrow \infty$.

Finally, we define rigid displacements in connected components $C_{i}^{k}$ of $B_{k} \backslash \bigcup_{i} E_{i}^{k}$ whose interior in the limit becomes empty, i.e., $C_{i}^{k}$ turns into an external filament. Recall that $\left|C_{i}^{k}\right| \rightarrow 0$ as $k \rightarrow \infty$. If $\mathcal{H}^{1}\left(\partial C_{i}^{k} \cap \Sigma\right)=0$, we define null-rigid displacement in $C_{i}^{k}$. If $\mathcal{H}^{1}\left(\partial C_{i}^{k} \cap \Sigma\right)>0$, then by condition (a6), $\partial C_{i}^{k}$ intersects the boundary of unique $S_{j_{i}}$, in which we have defined rigid displacement $a_{k}^{j_{i}}$. In this case we define the same $a_{k}^{j_{i}}$ in $C_{i}^{k}$ so that $\bigcup_{i} \partial C_{i}^{k} \cap J_{u_{k}+a_{k}^{j_{i}}} \subset J_{u_{k}}$, that is, we do not create extra jump set.

Let

$$
a_{k}:=\sum_{n} a_{k}^{n} \sum_{i \in I_{n}, j \in Y_{n}} \chi_{E_{i}^{k} \cup S_{j}}+\sum_{i \in I \backslash \cup_{n} I_{n}} \tilde{a}_{k}^{i} \chi_{E_{i}^{k}}+\sum_{i, \mathcal{H}^{1}\left(\partial C_{i}^{k} \cap \Sigma\right)>0} a_{k}^{j_{i}} \chi_{C_{i}^{k}}
$$

and

$$
u:=\sum_{n} w_{n} \sum_{i \in I_{n}, j \in Y_{n}} \chi_{E_{i} \cup S_{j}}+\sum_{i \in I \backslash \cup_{n} I_{n}} w^{i} \chi_{E_{i}}
$$


By construction, $a_{k}$ is a piecewise rigid displacement associated to $B_{k}, u \in$ $H_{\text {loc }}^{1}\left(\operatorname{Int}(A) \cup S ; \mathbb{R}^{2}\right)$ and $u_{k}+a_{k} \rightarrow u$ a.e. $\operatorname{in} \operatorname{Int}(A) \cup S$. Note that $e\left(u_{k}+a_{k}\right)=$ $e\left(u_{k}\right)$. Hence, by convergence $A_{k} \stackrel{\tau_{\mathcal{A}}}{\rightarrow} A$ and by (3.33), for any Lipschitz open set $D \subset \subset \operatorname{Int}(A) \cup S$,

$$
\int_{D}\left|e\left(u_{k}+a_{k}\right)\right|^{2} \mathrm{~d} x<\frac{R+\int_{\Sigma}|\beta| \mathrm{d} \mathcal{H}^{1}}{2 c_{3}}
$$

for all large $k$ (depending only $D$ ). Since $u_{k}+a_{k} \rightarrow u$ a.e. in $D$, by the PoincaréKorn inequality, $e\left(u_{k}+a_{k}\right) \rightarrow e(u)$ weakly in $L^{2}\left(D ; \mathbb{M}_{\text {sym }}^{2 \times 2}\right)$. Then by the convexity of $v \mapsto \int_{D}|e(v)|^{2} \mathrm{~d} x$, we get

$$
\int_{D}|e(u)|^{2} \mathrm{~d} x \leqq \liminf _{k \rightarrow \infty} \int_{D}\left|e\left(u_{k}+a_{k}\right)\right|^{2} \mathrm{~d} x \leqq \frac{R+\int_{\Sigma}|\beta| \mathrm{d} \mathcal{H}^{1}}{2 c_{3}} .
$$

Hence, letting $D \nearrow \operatorname{Int}(A) \cup S$ we get $u \in G S B D^{2}\left(\operatorname{Int}(A \cup S \cup \Sigma) ; \mathbb{R}^{2}\right)$. Consequently, $(A, u) \in \mathcal{C}_{m}$ and $\left(B_{k}, u_{k}+a_{k}\right) \stackrel{\tau_{\mathcal{C}}}{\rightarrow}(A, u)$ as $k \rightarrow \infty$.

We observe that if $S=\emptyset$, the terms of the surface energy $\mathcal{S}\left(A_{k}, u_{k}\right)$ related to $\Sigma$ disappears, and hence, using $e\left(u_{k}+a_{k}\right)=e\left(u_{k}\right)$ and property (a1),

$$
\mathcal{F}\left(A_{k}, u_{k}\right)=\mathcal{F}\left(B_{k}, u_{k}+a_{k}\right)+o(1),
$$

where $o(1) \rightarrow 0$ as $n \rightarrow \infty$, and so we can define $D_{n}=B_{n}$.

Step 2: Further modification of $\left\{B_{k}\right\}$. Without loss of generality we assume $\mathrm{V}<|\Omega|$. It remains to control $J_{u_{k}+a_{k}}$ at $\Sigma$ since, as mentioned above, adding different rigid displacements to $u_{k}$ in connected components of the substrate and the free crystal whose closures intersect can result in a larger jump set $J_{u_{k}+a_{k}}$ than $J_{u_{k}}$. Recall that by condition (a4) and (a6),

$$
\lim _{k \rightarrow \infty} \mathcal{H}^{1}\left(J_{u_{k}+a_{k}} \backslash \partial^{*} A\right)=0 .
$$

Hence, we need only to control $J_{u_{k}} \cap \partial^{*} A$. The idea here is to remove a "small" subset $R_{k}$ of $B_{k}$ containing almost all points $x \in \Sigma \cap \partial^{*} A \cap\left(\partial^{*} A_{k} \backslash J_{u_{k}}\right)$ which in the limit becomes jump for $u$. In order to keep volume constraint, we will insert a square $U_{k}$ of volume $\left|R_{k}\right|$ in $\Omega \backslash A_{k}$. This is possible since $\left|R_{k}\right| \rightarrow 0$ and $\left|A_{k}\right| \leqq \mathrm{V}$ for all $k$.

More precisely, we prove that for any $\delta \in(0,1 / 16)$, there exist $k_{\delta}>0$ and $\left(B_{k}^{\delta}, u_{k}^{\delta}\right) \in \mathcal{C}_{m}$ with $\left|B_{k}\right|=\left|B_{k}^{\delta}\right|$ such that $\left(B_{k}^{\delta}, u_{k}^{\delta}\right)$ and

$$
\mathcal{F}\left(B_{k}, u_{k}\right)+4 \delta\left(1+c_{2}\right) \mathcal{H}^{1}(\Sigma)+4 c_{2} \delta>\mathcal{F}\left(B_{k}^{\delta}, u_{k}+a_{k}\right)
$$

for any $k>k_{\delta}$.

We divide the proof into four steps.

Substep 2.1. By assumptions of the theorem, conditions (a2) and (a5) and Lemma 3.2 (b), $|A| \leqq \mathrm{v}$. Hence, we can choose a square $U \subset \subset \Omega \backslash \bar{A}$. By (a2) and the definition of $\tau_{\mathcal{A}}$-convergence, there is no loss of generality in assuming $U \subset \subset \Omega \backslash \overline{B_{k}}$ for any $k$. Let

$$
\epsilon_{0}:=\sqrt{|U|}
$$


Without loss of generality, we assume $\epsilon_{0} \in\left(0, \frac{1}{2}\right)$.

First we observe that for any $\delta \in(0,1)$ :

(b1) since $\Sigma$ is Lipschitz, for $\mathcal{H}^{1}$-a.e. $x \in \Sigma$, there exist a unit normal $v_{\Sigma}(x)$ to $\Sigma$ and $\overline{r_{x}}>0$ such that for any $r \in\left(0, \overline{r_{x}}\right), U_{r, v_{\Sigma}(x)}(x) \cap \Sigma$ can be represented as a graph of a Lipschitz function over tangent line $U_{r, v_{\Sigma}(x)}(x) \cap T_{x}$ at $x$ in the direction $v_{\Sigma}(x)$

(b2) since $\varphi$ is uniformly continuous, for any $x \in \bar{\Omega}$ there exists $\overline{r_{x}^{\delta}}>0$ such that for any $y \in U_{r_{x}^{\delta}, v_{\Sigma}(x)}(x)$ and $\xi \in \mathbb{S}^{1}$,

$$
|\varphi(y, \xi)-\varphi(x, \xi)|<\delta
$$

(b3) since $\mathcal{H}^{1}$-a.e. $x \in \Sigma$ is the Lebesgue point of $\beta$, there exists $\overline{r_{x}^{\delta}}>0$ such that for any $r \in\left(0, \overline{r_{x}^{\delta}}\right)$,

$$
\int_{U_{r, \nu_{\Sigma}(x)}(x) \cap \Sigma}|\beta(y)-\beta(x)| \mathrm{d} \mathcal{H}^{1}(y)<\delta \mathcal{H}^{1}\left(U_{r, \nu_{\Sigma}(x)}(x) \cap \Sigma\right) ;
$$

(b4) for $\mathcal{H}^{1}$-a.e. $x \in \Sigma \cap \partial^{*} A$ one has

$$
\theta^{*}(\Sigma, x)=\theta_{*}(\Sigma, x)=\theta^{*}\left(\Sigma \cap \partial^{*} A, x\right)=\theta_{*}\left(\Sigma \cap \partial^{*} A, x\right)=1,
$$

thus, there exists $r_{x}>0$ such that for any $r \in\left(0, r_{x}\right)$,

$$
\mathcal{H}^{1}\left(U_{r, v_{\Sigma}(x)}(x) \cap\left(\Sigma \backslash \partial^{*} A\right)\right)<2 \delta r ;
$$

(b5) by Proposition A.4 applied with a connected component of $\partial A$, for a.e. $x \in$ $\Sigma \cap \partial^{*} A, \overline{U_{1, v_{\Sigma}(x)}(x)} \cap \sigma_{\rho, x}(\partial A) \stackrel{K}{\longrightarrow} \overline{U_{1, v_{\Sigma}(x)}(0)} \cap\left(T_{x}-x\right)$ as $\rho \rightarrow 0$, where $\sigma_{\rho, x}(y):=\frac{y-x}{\rho}$ is the blow up map and $T_{x}-x$ is the straight line passing through the origin and parallel to the tangent line $T_{x}$ of $\partial^{*} A$ (and of $\Sigma)$ at $x$. Thus, there exists $\overline{r_{x}^{\delta}}>0$ such that for any $r \in\left(0, \overline{r_{x}^{\delta}}\right), U_{r, v_{\Sigma}(x)}(x) \cap$ $\left\{\operatorname{dist}\left(\cdot, T_{x}\right)>\delta r\right\} \subset \operatorname{Int}(A) \cup S$.

By (3.35), there exists $\bar{k}_{\delta}>0$ such that

$$
\mathcal{H}^{1}\left(J_{u_{k}+a_{k}} \backslash \partial^{*} A\right)<\delta
$$

for any $k>\bar{k}_{\delta}$.

Fix $\delta \in\left(0, \frac{1}{16}\right)$ and let $t_{\delta}>0$ be such that

$$
\left|\left\{x \in \Omega: \operatorname{dist}(x, \Sigma)<t_{\delta}\right\}\right|<\delta^{2} \epsilon_{0}^{2},
$$

where $\epsilon_{0}$ is given in (3.37).

We now consider connected components $E_{i}$ and $S_{j}$ such that the associated rigid displacements are different. Let $I^{\prime}$ be the set of all $i \in I$ such that $\mathcal{H}^{1}\left(\partial^{*} E_{i} \cap \partial S\right)>$ 0 and $\liminf _{k \rightarrow \infty} \mathcal{H}^{1}\left(\partial E_{i}^{k} \cap \partial S\right)>0$, and there exists a connected component $S_{j}$ of $S$ such that $u_{k}+b_{k}^{i} \rightarrow u$ a.e. in $E_{i}$ and $\left|u_{k}+b_{k}^{i}\right| \rightarrow+\infty$ a.e. in $S_{j}$ for the associated sequence $\left\{b_{k}^{i}\right\}$ of rigid displacements in $E_{i}$. 
Set

$$
L:=\Sigma \cap \bigcup_{i \in I^{\prime}} \partial^{*} E_{i}
$$

Note that $\bar{L} \subset \Sigma$ and by (b1)-(b5), for a.e. $x \in \bar{L}$ for which $v_{\Sigma}(x)$ and $v_{A}(x)$ exist and $v_{\Sigma}(x)=v_{A}(x)$ there is

$$
r_{x}:=r_{x}^{\delta} \in\left(0, \frac{t_{\delta}}{8}\right)
$$

such that properties (b1)-(b5) holds with $x$ and $r=r_{x}$. Note that for any such $x$ :

(c1) since $B_{k} \stackrel{\tau_{\mathcal{A}}}{\rightarrow} A$, by property (b5), there exists $\overline{k_{x}^{\delta}}>\bar{k}_{\delta}$ such that $U_{r, v_{\Sigma}(x)}(x) \cap$ $\left\{\operatorname{dist}\left(\cdot, T_{x}\right)>\delta r\right\} \subset \operatorname{Int}\left(B_{k}\right) \cup S$ for any $k>\frac{x}{k_{x}^{\delta}}$;

(c2) by Proposition 3.9 applied with $u_{k}+a_{k}^{i}$, there exists $\overline{k_{x}^{\delta}}>\bar{k}_{\delta}$ such that

$$
\begin{aligned}
& \int_{U_{r, \nu_{0}}(x) \cap \Omega \cap \partial^{*} A_{k}} \varphi\left(y, v_{A_{k}}\right) \mathrm{d} \mathcal{H}^{1}+2 \int_{U_{r, v_{0}}(x) \cap \Omega \cap\left(A_{k}^{(0)} \cup A_{k}^{(1)}\right) \cap \partial A_{k}} \varphi\left(y, v_{A_{k}}\right) \mathrm{d} \mathcal{H}^{1} \\
& \quad+\int_{U_{r, v_{0}}(x) \cap \Sigma \cap A_{k}^{(0)} \cap \partial A_{k}}\left(\varphi\left(y, v_{\Sigma}\right)+\beta\right) \mathrm{d} \mathcal{H}^{1} \\
& \quad+\int_{U_{r, v_{0}}(x) \cap \Sigma \cap \partial^{*} A_{k} \backslash J_{u_{k}}} \beta \mathrm{d} \mathcal{H}^{1}+\int_{U_{r, v_{0}}(x) \cap J_{u_{k}}} \varphi\left(y, v_{\Sigma}\right) \mathrm{d} \mathcal{H}^{1} \\
& \geq \frac{1}{1+\frac{\delta}{c_{2}}} \int_{U_{r, v_{0}}(x) \cap \Sigma \cap \partial^{*} A} \varphi\left(y, v_{\Sigma}\right) \mathrm{d} \mathcal{H}^{1}-\delta \int_{U_{r, v_{0}}(x) \cap \Sigma} \varphi\left(y, v_{\Sigma}\right) \mathrm{d} \mathcal{H}^{1} .
\end{aligned}
$$

for any $k>\overline{k_{x}^{\delta}}$, where $r:=r_{x}$ and $\nu_{0}:=v_{\Sigma}(x)$.

Substep 2.2. Let $x \in L$ be with properties (c1)-(c2) and let $U_{r}:=U_{r, v_{\Sigma}(x)}(x)$ and $Q_{x} \subset \Omega \cap$ be the open set whose boundary consists of $\Gamma_{1}:=U_{r} \cap \Sigma$, two segments $\Gamma_{2}, \Gamma_{3} \subset \partial U_{r}$ of length at most $2 \delta r$, parallel to $v_{\Sigma}(x)$, and the segment $\Gamma_{4}:=\Omega \cap U_{r} \cap\left\{\operatorname{dist}\left(\cdot, T_{x}\right)=\delta\right\}$ of length $2 r$. Given $k>k_{x}^{\delta}$ let

$$
\left.\widehat{B}_{k}^{\delta}:=B_{k} \backslash Q_{x}\right) \cup\left(\bar{\Omega} \cap \partial U_{r}\right) .
$$

Clearly, $\left(\widehat{B}_{k}^{\delta}, u_{k}+a_{k}\right) \in \mathcal{A}_{m}$. We claim that

$$
\mathcal{S}\left(B_{k}, u_{k}\right) \geqq \mathcal{S}\left(\widehat{B}_{k}^{\delta}, u_{k}\right)-4 \delta\left(1+c_{2}\right) \mathcal{H}^{1}\left(U_{r} \cap \Sigma\right) .
$$

Indeed, without loss of generality, we assume that $x=0$ and $\nu_{\Sigma}(x)=\mathbf{e}_{2}$. By the anisotropic minimality of segments,

$$
\int_{\Gamma_{1}} \varphi\left(0, v_{\Sigma}\right) \mathrm{d} \mathcal{H}^{1}+\int_{\Gamma_{2} \cup \Gamma_{3}} \varphi\left(0, \mathbf{e}_{2}\right) \mathrm{d} \mathcal{H}^{1} \geqq \int_{\Gamma_{4}} \varphi\left(0, \mathbf{e}_{2}\right) \mathrm{d} \mathcal{H}^{1} .
$$

Since $\mathcal{H}^{1}\left(\Gamma_{1}\right) \geqq 2 r=\mathcal{H}^{1}\left(\Gamma_{4}\right)$ and $\mathcal{H}^{1}\left(\Gamma_{2}\right), \mathcal{H}^{1}\left(\Gamma_{3}\right) \leqq 2 \delta r$ and, also by property (b2), we have

$$
\int_{\Gamma_{1}} \varphi\left(0, v_{\Sigma}\right) \mathrm{d} \mathcal{H}^{1} \leqq \int_{\Gamma_{1}} \varphi\left(y, v_{\Sigma}\right) \mathrm{d} \mathcal{H}^{1}+\delta \mathcal{H}^{1}\left(\Gamma_{1}\right),
$$


and

$$
\int_{\Gamma_{2} \cup \Gamma_{3}} \varphi\left(0, \mathbf{e}_{2}\right) \mathrm{d} \mathcal{H}^{1} \leqq 2 \delta \varphi\left(0, \mathbf{e}_{2}\right) \mathcal{H}^{1}\left(\Gamma_{1}\right)
$$

and

$$
\int_{\Gamma_{4}} \varphi\left(0, \mathbf{e}_{2}\right) \mathrm{d} \mathcal{H}^{1} \geqq \int_{\Gamma_{4}} \varphi\left(y, \mathbf{e}_{2}\right) \mathrm{d} \mathcal{H}^{1}-\delta \mathcal{H}^{1}\left(\Gamma_{1}\right),
$$

hence, using (2.4) in (3.45), from (3.43) and (3.44)-(3.46) we obtain

$$
\int_{\Gamma_{4}} \varphi\left(y, \mathbf{e}_{2}\right) \mathrm{d} \mathcal{H}^{1} \leqq \int_{\Gamma_{1}} \varphi\left(y, v_{\Sigma}\right) \mathrm{d} \mathcal{H}^{1}+2 \delta\left(1+c_{2}\right) \mathcal{H}^{1}\left(\Gamma_{1}\right)
$$

Denoting by $\alpha_{k}$ the left-hand side of (3.41), by condition (a1) and the definition of $\widehat{B}_{k}^{\delta}$, we have

$$
\begin{aligned}
\mathcal{S}\left(B_{k}, u_{k}\right)-\mathcal{S}\left(\widehat{B}_{k}^{\delta}, u_{k}\right) \geqq & \alpha_{k}-2 \int_{\partial B_{k} \backslash \partial A_{k}} \varphi\left(y, v_{B_{k}}\right) \mathrm{d} \mathcal{H}^{1} \\
& -\int_{\Gamma_{2} \cup \Gamma_{3} \cup \Gamma_{4}} \phi\left(y, v_{\left.B_{k}^{\delta}\right)} \mathrm{d} \mathcal{H}^{1}-\int_{\left(B_{k}^{\delta}\right)^{(0)} \cap\left(\Gamma_{2} \cup \Gamma_{3}\right)} \phi\left(y, v_{\left.B_{k}^{\delta}\right)} \mathrm{d} \mathcal{H}^{1},\right.\right.
\end{aligned}
$$

thus, using $\Gamma_{1}=U_{r} \cap \Sigma$, from (3.41), (3.47), (3.45), (3.38) and (2.4) we obtain

$$
\begin{aligned}
\mathcal{S}\left(B_{k}, u_{k}\right)-\mathcal{S}\left(\widehat{B}_{k}^{\delta}, u_{k}\right) \geqq & \frac{1}{1+\frac{\delta}{c_{2}}} \int_{U_{r} \cap \Sigma \cap \partial^{*} A} \varphi\left(y, v_{\Sigma}\right) \mathrm{d} \mathcal{H}^{1}-\int_{U_{r} \cap \Sigma} \varphi\left(y, v_{\Sigma}\right) \mathrm{d} \mathcal{H}^{1} \\
& -3 \delta\left(1+c_{2}\right) \mathcal{H}^{1}\left(U_{r} \cap \Sigma\right) .
\end{aligned}
$$

In the last inequality using $\frac{1}{1+\frac{\delta}{c_{2}}} \geqq 1-\frac{\delta}{c_{2}}$ and inequality (2.4) once more we deduce

$$
\begin{aligned}
\mathcal{S}\left(B_{k}, u_{k}\right) \geqq & \mathcal{S}\left(\widehat{B}_{k}^{\delta}, u_{k}\right)-\int_{U_{r} \cap\left(\Sigma \backslash \partial^{*} A\right)} \varphi\left(y, v_{\Sigma}\right) \mathrm{d} \mathcal{H}^{1} \\
& -\delta\left(4+3 c_{2}\right) \mathcal{H}^{1}\left(U_{r} \cap \Sigma\right) .
\end{aligned}
$$

Now condition (b4) and (2.4) and the inequality $\mathcal{H}^{1}\left(\Gamma_{1}\right) \geqq 2 r$ imply

$\int_{U_{r} \cap\left(\Sigma \backslash \partial^{*} A\right)} \varphi\left(y, v_{\Sigma}\right) \mathrm{d} \mathcal{H}^{1} \leqq c_{2} \mathcal{H}^{1}\left(U_{r} \cap\left(\Sigma \backslash \partial^{*} A\right)\right) \leqq 2 \delta c_{2} r \leqq \delta c_{2} \mathcal{H}^{1}\left(U_{r} \cap \Sigma\right)$.

Inserting this in (3.48) we get (3.42).

Substep 2.3. Now we choose finitely many points $x_{1} \ldots, x_{N} \in \bar{L}$ with corresponding $r_{1}, \ldots, r_{N}$ satisfying (b1)-(b5) and (c1)-(c2) such that the squares $\left\{U_{r_{j}, v_{\Sigma}\left(x_{j}\right)}\left(x_{j}\right)\right\}_{j=1}^{N}$ are pairwise disjoint and

$$
\mathcal{H}^{1}\left(L \backslash \bigcup_{j=1}^{N} U_{r_{j}, v_{\Sigma}\left(x_{j}\right)}\left(x_{j}\right)\right)<\delta
$$


Recalling the definition of $k_{\delta}^{x}$ in condition (c2) and the definition $\bar{k}_{\delta}$ in (3.38), let $k_{\delta}:=\max \left\{\bar{k}_{\delta}, \overline{k_{\delta}^{x_{1}}}, \ldots, \overline{k_{\delta}^{x_{N}}}\right\}$ and let $Q_{x_{j}} \subset \Omega \cap U_{r_{j}, v_{\Sigma}\left(x_{j}\right)}\left(x_{j}\right)$ be as in Substep 2.2. Set

$$
\widetilde{B}_{k}^{\delta}:=\left(B_{k} \backslash \bigcup_{j=1}^{N} Q_{x_{j}}\right) \cup \bigcup_{j=1}^{N}\left(\bar{\Omega} \cap \partial U_{r_{j}, \nu_{\Sigma}\left(x_{j}\right)}\left(x_{j}\right)\right) .
$$

Then, as in the proof of (3.42),

$$
\mathcal{S}\left(B_{k}, u_{k}\right)-\mathcal{S}\left(\widetilde{B}_{k}^{\delta}, u_{k}\right) \geqq-4 \delta\left(1+c_{2}\right) \sum_{j=1}^{N} \mathcal{H}^{1}\left(U_{r_{j}, \nu_{\Sigma}\left(x_{j}\right)}\left(x_{j}\right) \cap \Sigma\right),
$$

so that by the pairwise disjointness of $\left\{U_{r_{j}, \nu_{\Sigma}\left(x_{j}\right)}\left(x_{j}\right)\right\}$,

$$
\mathcal{S}\left(B_{k}, u_{k}\right)-\mathcal{S}\left(\widetilde{B}_{k}^{\delta}, u_{k}\right) \geqq-4 \delta\left(1+c_{2}\right) \mathcal{H}^{1}(\Sigma) .
$$

Recalling (3.49) and (3.38), and using (2.4), we estimate

$$
\begin{aligned}
& \mathcal{S}\left(\widetilde{B}_{k}^{\delta}, u_{k}+a_{k}\right)-\mathcal{S}\left(\widetilde{B}_{k}^{\delta}, u_{k}\right) \\
& \quad \leqq \int_{L \backslash \bigcup_{j} U_{r_{j}, v_{\Sigma}\left(x_{j}\right)}\left(x_{j}\right)} \varphi\left(y, v_{\Sigma}\right) \mathrm{d} \mathcal{H}^{1}+\int_{J_{u_{k}+a_{k} \backslash \partial^{*} A}} \varphi\left(y, v_{\Sigma}\right) \mathrm{d} \mathcal{H}^{1}<2 c_{2} \delta,
\end{aligned}
$$

hence, from (3.50), we get

$$
\mathcal{S}\left(B_{k}, u_{k}\right)-\mathcal{S}\left(\widetilde{B}_{k}^{\delta}, u_{k}+a_{k}\right) \geqq-4 \delta\left(1+c_{2}\right) \mathcal{H}^{1}(\Sigma)-2 c_{2} \delta .
$$

On the other hand, since $\operatorname{Int}\left(\widetilde{B}_{k}^{\delta}\right) \subset \operatorname{Int}\left(B_{k}\right)$ and $e\left(u_{k}\right)=e\left(u_{k}+a_{k}\right)$,

$$
\mathcal{W}\left(B_{k}, u_{k}\right) \geqq \mathcal{W}\left(\widetilde{B}_{k}^{\delta}, u_{k}+a_{k}\right) .
$$

From (3.51) and (3.52) we deduce

$$
\mathcal{F}\left(B_{k}, u_{k}\right) \geqq \mathcal{F}\left(\widetilde{B}_{k}^{\delta}, u_{k}+a_{k}\right)-4 \delta\left(1+c_{2}\right) \mathcal{H}^{1}(\Sigma)-2 c_{2} \delta .
$$

However, by construction, $\left|B_{k}\right| \geqq\left|\widetilde{B}_{k}^{\delta}\right|$ since $\widetilde{B}_{k}^{\delta} \subset B_{k} \cup \bigcup_{j=1}^{N}\left(\bar{\Omega} \cap \partial U_{r_{j}, v_{\Sigma}\left(x_{j}\right)}\left(x_{j}\right)\right)$. Thus, $R_{k}:=B_{k} \backslash \widetilde{B}_{k}^{\delta}$ satisfies $\left|R_{k}\right|=\left|B_{k} \Delta \widetilde{B}_{k}^{\delta}\right|$. Since $\bigcup_{j} Q_{x_{j}} \subset \Omega \cap\{\operatorname{dist}(\cdot, \Sigma)<$ $\left.\frac{t_{\delta}}{8}\right\}$, thus, by (3.39), $\left|R_{k}\right|<\delta^{2} \epsilon_{0}$. Hence, we choose a square $U_{k} \subset U$ (see (3.37)) such that $\left|U_{k}\right|=\left|R_{k}\right|$. For $k>k_{\delta}$ set

$$
B_{k}^{\delta}:=\widetilde{B}_{k}^{\delta} \cup U_{k}
$$

In order not to increase the number of connected components of $B_{k}^{\delta}$, we translate $U_{k}$ in $\Omega \backslash \overline{B_{k}}$ until it touches to $\partial \widetilde{B}_{k}^{\delta}$. Define

$$
u_{k}^{\delta}:=u_{k} \chi_{\widetilde{B}_{k}^{\delta}}+u_{0} \chi_{U_{k}}
$$


Then $\left\{\left(B_{k}^{\delta}, u_{k}^{\delta}\right)\right\} \subset \mathcal{C}_{m}$ and for any $k>k_{\delta}$ by (3.51) and (3.52)

$$
\mathcal{F}\left(B_{k}, u_{k}\right) \geqq \mathcal{F}\left(B_{k}^{\delta}, u_{k}^{\delta}\right)-\mathcal{S}\left(U_{k}, u_{0}\right)-4 \delta\left(1+c_{2}\right) \mathcal{H}^{1}(\Sigma)-2 c_{2} \delta .
$$

By the choice of $U_{k}$, its sidelength is less that $\delta \epsilon_{0}$, hence, using $\epsilon_{0}<\frac{1}{2}$ and (2.4), $\mathcal{S}\left(U_{k}, u_{0}\right) \leqq 2 c_{2} \delta$ so that

$$
\mathcal{F}\left(B_{k}, u_{k}\right) \geqq \mathcal{F}\left(B_{k}^{\delta}, u_{k}^{\delta}\right)-4 \delta\left(1+c_{2}\right) \mathcal{H}^{1}(\Sigma)-4 c_{2} \delta .
$$

Step 3: Construction of $\left(D_{n}, v_{n}\right)$. Notice that the sequence $\left\{\left(B_{k}^{\delta}, u_{k}^{\delta}\right)\right\}$ in general does not need to satisfy $B_{k}^{\delta} \stackrel{\tau_{\mathcal{A}}}{\rightarrow} A$, since we removed "something" from $B_{k}$ and added a square $U_{k}$. To overcome this problem, we take $\delta=\delta_{n}:=\frac{1}{16 n}$ and $\left(D_{n}, v_{n}\right):=\left(B_{k_{n}}^{\delta_{n}}, u_{k_{n}}^{\delta_{n}}\right)$, where $k_{n}:=k_{\delta_{n}}+1$, and there is no loss of generality in assuming $n \mapsto k_{n}$ is increasing. Denote $r_{j}^{n}:=r_{x_{j}}^{\delta_{n}}$, where the latter is defined in Substep 2.3 and notice that by (3.39) and (3.40) $r_{j}^{n} \rightarrow 0$ as $n \rightarrow \infty$ In particular, $\partial D_{n} \stackrel{K}{\rightarrow} \partial A$ as $n \rightarrow \infty$. Thus, $D_{n} \stackrel{\tau_{\mathcal{A}}}{\rightarrow} A$. Since $\left|D_{n} \Delta A\right| \rightarrow 0, v_{n} \rightarrow u$ a.e. in $A \cup S$. By (3.36)

$$
\mathcal{F}\left(B_{k_{n}}, u_{k_{n}}\right)+\frac{\left(1+c_{2}\right) \mathcal{H}^{1}(\Sigma)+c_{2}}{4 n} \geqq \mathcal{F}\left(D_{n}, u_{n}\right),
$$

thus (2.9) follows from (3.53) and (3.34).

\section{Lower Semicontinuity}

In this section we consider more general surface energies. For every $A \in \mathcal{A}$ and $J_{A} \in \mathcal{J}_{A}$, where

$$
\mathcal{J}_{A}:=\left\{J \subseteq \Sigma \cap \overline{\partial^{*} A}: \quad J \text { is } \mathcal{H}^{1}-\text { measurable }\right\}
$$

is the collection of all possible delaminations on $\Sigma$, we define

$$
\begin{aligned}
\mathcal{S}\left(A, J_{A} ; \varphi, g\right):= & \int_{\Omega \cap \partial^{*} A} \varphi\left(x, v_{A}\right) \mathrm{d} \mathcal{H}^{1}+2 \int_{\Omega \cap\left(A^{(1)} \cup A^{(0)}\right) \cap \partial A} \varphi\left(x, v_{A}\right) \mathrm{d} \mathcal{H}^{1} \\
& +\int_{\Sigma \backslash \partial A} g(x, 0) \mathrm{d} \mathcal{H}^{1}+\int_{\Sigma \cap A^{(0)} \cap \partial A}\left(\varphi\left(x, v_{\Sigma}\right)+g(x, 1)\right) \mathrm{d} \mathcal{H}^{1} \\
& +\int_{\Sigma \cap \partial^{*} A \backslash J_{A}} g(x, 1) \mathrm{d} \mathcal{H}^{1}+\int_{J_{A}}\left(\varphi\left(x, v_{\Sigma}\right)+g(x, 0)\right) \mathrm{d} \mathcal{H}^{1},
\end{aligned}
$$

where $g: \Sigma \times\{0,1\} \rightarrow \mathbb{R}$ is a Borel function. We remark that $\mathcal{S}(A, u)=$ $\mathcal{S}\left(A, J_{u} ; \varphi, g\right)$ with $g(x, s)=\beta(x) s$ and $J_{A}=J_{u}$.

The main result of this section is the following.

Proposition 4.1. (Lower-semicontinuity of $\mathcal{S}$ ) Suppose that $g: \Sigma \times\{0,1\} \rightarrow \mathbb{R}$ is a Borel function such that $g(\cdot, s) \in L^{1}(\Sigma)$ for $s=0,1$ and

$$
|g(x, 1)-g(x, 0)| \leqq \varphi\left(x, v_{\Omega}(x)\right)
$$

for $\mathcal{H}^{1}$-a.e. $x \in \Sigma$. Let $A_{k} \in \mathcal{A}_{m}, J_{A_{k}} \in \mathcal{J}_{A_{k}}, A \in \mathcal{A}_{m}$ and $J_{A} \in \mathcal{J}_{A}$ be such that 
(a) $A_{k} \stackrel{\tau_{\mathcal{A}}}{\rightarrow}$ A as $k \rightarrow \infty$;

(b) $\mathcal{H}^{1}$-a.e. $x \in J_{A}$ there exist $r=r_{x}>0, w, w_{k} \in G S B D^{2}\left(B_{r}(x) ; \mathbb{R}^{2}\right)$ and relatively open subset $L_{k}$ of $\Sigma$ with $\mathcal{H}^{1}\left(L_{k}\right)<1 / k$ for which

$$
\left\{\begin{array}{l}
J_{w_{k}} \subset B_{r}(x) \cap\left(J_{A_{k}} \cup\left(\Omega \cap \partial A_{k}\right) \cup L_{k}\right) \text { and } J_{A} \subset J_{w} \\
w_{k} \rightarrow w \text { a.e. in } B_{r}(x) \text { as } k \rightarrow \infty \\
\sup _{k \geqq 1} \int_{B_{r}(x)}\left|e\left(w_{k}\right)\right|^{2} d x<\infty
\end{array}\right.
$$

Then

$$
\liminf _{k \rightarrow \infty} \mathcal{S}\left(A_{k}, J_{A_{k}} ; \varphi, g\right) \geqq \mathcal{S}\left(A, J_{A} ; \varphi, g\right) .
$$

We prove Proposition 4.1 using a blow-up around the points of the boundary of $A$. Given $y_{o} \in \mathbb{R}^{2}$ and $\rho>0$, the blow-up map $\sigma_{\rho, y_{o}}: \mathbb{R}^{2} \rightarrow \mathbb{R}^{2}$ is defined as

$$
\sigma_{\rho, y_{o}}(y):=\frac{y-y_{o}}{\rho}
$$

When $y_{o}=0$ we write $\sigma_{\rho}$ instead of $\sigma_{\rho, 0}$. Given $v \in \mathbb{S}^{1}, U_{\rho, v}(x)$ is an open square of sidelength $2 \rho>0$ centered at $x$ whose sides are either perpendicular or parallel to $v$; if $v=\mathbf{e}_{2}$ and $x=0$, we write $U_{\rho, v}(0):=U_{\rho}=(-\rho, \rho)^{2}, U_{\rho}^{+}=$ $(-\rho, \rho) \times(0, \rho)$, and $I_{\rho}:=[-\rho, \rho] \times\{0\}$. Observe that $\sigma_{\rho, x}\left(U_{\rho, v}(x)\right)=U_{1, v}(0)$ and $\sigma_{\rho, x}\left(\overline{U_{\rho, v}(x)}\right)=\overline{U_{1, v}(0)}$. We denote by $\pi$ the projection onto $x_{1}$-axis i.e.,

$$
\pi(x)=\left(x_{1}, 0\right)
$$

The following auxiliary results will be used in the proof of Proposition 4.1:

Lemma 4.2. Let $U$ be any open square, $K \subset \bar{U}$ be a nonempty closed set and $E_{k} \subset U$ be such that $\operatorname{sdist}\left(\cdot, \partial E_{k}\right) \stackrel{k \rightarrow \infty}{\longrightarrow} \operatorname{dist}(\cdot, K)$ uniformly in $\bar{U}$. Then $E_{k} \stackrel{\mathcal{K}}{\rightarrow} K$ as $k \rightarrow \infty$. Analogously if $\operatorname{sdist}\left(\cdot, \partial E_{k}\right) \stackrel{k \rightarrow \infty}{\longrightarrow}-\operatorname{dist}(\cdot, K)$ uniformly in $\bar{U}$, then $\bar{U} \backslash E_{k} \stackrel{\mathcal{K}}{\rightarrow} K$ as $k \rightarrow \infty$.

Proof. We prove only the first assertion, the second being the same. If $x_{k} \in E_{k}$ is such that $x_{k} \rightarrow x$, then by assumption,

$$
\operatorname{dist}(x, K)=\lim _{k \rightarrow \infty} \operatorname{sdist}\left(x_{k}, \partial E_{k}\right) \leqq 0
$$

so that $x \in K$. On the other hand, given $x \in K$ suppose that there exists $r>0$ such that $B_{r}(x) \cap E_{k}=\emptyset$ for infinitely many $k$. Then for $\operatorname{such} k, \operatorname{sdist}\left(x, \partial E_{k}\right)=$ $\operatorname{dist}\left(x, E_{k}\right) \geqq r>0$, which contradicts to the assumption.

In the next lemma we observe that the endpoints of every curve $\Gamma$ contained in the boundary of any bounded set $A$ with connected boundary are still arcwise connected if we remove the boundary of $\operatorname{Int}(\bar{A})$ belonging to $\Gamma$. 
Lemma 4.3. Let $A \subset \mathbb{R}^{2}$ be a bounded set such that $\partial A$ is connected and has finite $\mathcal{H}^{1}$ measure. Suppose that $x, y \in \partial A$ are such that $x \neq y$ and $\Gamma \subset \partial A$ is a curve connecting $x$ to $y$. Then there exists a curve $\Gamma^{\prime} \subset \overline{\partial A \backslash(\Gamma \cap \partial \operatorname{Int}(\bar{A}))}$ connecting $x$ to $y$.

Proof. Without loss of generality we assume $G:=\operatorname{Int}(\bar{A}) \neq \emptyset$, otherwise we simply take $\Gamma^{\prime}=\Gamma$. Note that

$$
\partial G=\left\{x \in \partial A: B_{r}(x) \cap \operatorname{Int}(A), B_{r}(x) \backslash \bar{A} \neq \emptyset \text { for every } r>0\right\} .
$$

Since connected compact sets of finite length are arcwise connected (see Proposition A.2), it suffices to show that $x$ and $y$ belong to the same connected component of $\overline{\partial A \backslash(\Gamma \cap \partial G)}$. Suppose that there exist two open sets $P, Q \subset \mathbb{R}^{2}$ with disjoint closures such that

$$
\overline{\partial A \backslash(\Gamma \cap \partial G)}=(P \cap \overline{\partial A \backslash(\Gamma \cap \partial G)}) \cup(Q \cap \overline{\partial A \backslash(\Gamma \cap \partial G)}),
$$

where $x \in P \cap \overline{\partial A \backslash(\Gamma \cap \partial G)}$ and $y \in Q \cap \overline{\partial A \backslash(\Gamma \cap \partial G)}$. Then $\Gamma \backslash \overline{P \cup Q} \neq \varnothing$ and

$$
\Gamma \backslash \overline{P \cup Q}=\partial G \backslash \overline{P \cup Q} .
$$

Since $\bar{P} \cap \bar{Q}=\emptyset$ and $\mathcal{H}^{1}(\Gamma)<\infty$, the number of connected components $\left\{L_{i}\right\}_{i=1}^{n}$ of $\Gamma \backslash \overline{P \cup Q}$ connecting both $P$ and $Q$ is at most finite. Moreover, since $\Gamma$ has no self-intersections (see Section A.2 for the definition of the curve in our setting) and the endpoints of $\Gamma$ belong to $P$ and $Q$, respectively, $n$ must be odd. However, by (4.8) $L_{i} \subset \partial G$, and hence, by (4.6), every neighborhood of $L_{i}$ contains points belonging to both $\operatorname{Int}(A)$ and $\mathbb{R}^{2} \backslash \bar{A}$. We reached a contradiction since in this case $\operatorname{Int}(A)$ would be unbounded.

Notice that if $A \in \mathcal{A}_{m}$, then $\overline{\partial^{*} A}=\partial A^{(1)}=\partial \operatorname{Int}(\bar{A})$.

Lemma 4.4. (Creation of external filament energy) Let $\phi$ be a norm in $\mathbb{R}^{2}$ satisfying

$$
c_{1} \leqq \phi(v) \leqq c_{2}, \quad v \in \mathbb{S}^{1}
$$

for some $c_{2} \geqq c_{1}>0$, and let $\left\{E_{k}\right\}$ be a sequence of subsets of $U_{1}$ such that

(a) $E_{k} \stackrel{\mathcal{K}}{\rightarrow} I_{1}$ as $k \rightarrow \infty$;

(b) there exists $m_{o} \in \mathbb{N}_{0}$ such that the number of connected components of $\partial E_{k}$ lying strictly inside $U_{1}$ does not exceed $m_{o}$.

Then, for every $\delta \in(0,1)$, there exists $k_{\delta}>1$ such that for any $k>k_{\delta}$,

$\int_{U_{1} \cap \partial^{*} E_{k}} \phi\left(v_{E_{k}}\right) d \mathcal{H}^{1}+2 \int_{U_{1} \cap\left(E_{k}^{(1)} \cup E_{k}^{(0)}\right) \cap \partial E_{k}} \phi\left(v_{E_{k}}\right) d \mathcal{H}^{1} \geqq 2 \int_{I_{1}} \phi\left(\mathbf{e}_{2}\right) d \mathcal{H}^{1}-\delta$. 
Proof. Let us denote the left hand side of (4.10) by $\alpha_{k}$. We may suppose $\sup _{k} \alpha_{k}<$ $\infty$. By assumption (a), for every $\delta \in(0,1)$ there exists $k_{1, \delta}>1$ such that

$$
E_{k} \subset[-1,1] \times\left(-\frac{\delta}{16 c_{2} m_{o}}, \frac{\delta}{16 c_{2} m_{o}}\right)
$$

for all $k>k_{1, \delta}$.

Step 1. Assume that for some $k>k_{1, \delta}, \partial E_{k}$ has a connected component $K^{1}$ intersecting both $\left\{x_{1}=1\right\}$ and $\left\{x_{1}=-1\right\}$. In this case by Lemma 4.3, $\overline{\partial E_{k} \backslash\left(K^{1} \cap \partial \operatorname{Int}(\bar{A})\right)}$ is also connected and contains a path $K^{2}$ connecting $\left\{x_{1}=1\right\}$ to $\left\{x_{1}=-1\right\}$. Note that $K^{1}$ and $K^{2}$ may coincide on $\left(E_{k}^{(1)} \cup E_{k}^{(0)}\right) \cap \partial E_{k}$. Let $R_{i}^{1}$ and $R_{i}^{2}, i=1,2$, be the segments along the vertical lines $\left\{x_{1}= \pm 1\right\}$ connecting the endpoints of $K^{1}$ and $K^{2}$ to $( \pm 1,0)$, respectively. Since $K^{1} \cap \partial^{*} E_{k}$ and $K^{2} \cap \partial^{*} E_{k}$ are disjoint up to a $\mathcal{H}^{1}$-negligible set

$$
\begin{aligned}
\alpha_{k} & \geqq \sum_{j=1}^{2}\left(\int_{K^{j} \cap \partial^{*} E_{k}} \phi\left(v_{E_{k}}\right) \mathrm{d} \mathcal{H}^{1}+\int_{K^{j} \backslash \partial^{*} E_{k}} \phi\left(v_{E_{k}}\right) \mathrm{d} \mathcal{H}^{1}\right) \\
& =\sum_{j=1}^{2} \int_{\gamma_{j}} \phi\left(v_{\gamma_{j}}\right) \mathrm{d} \mathcal{H}^{1}-\sum_{i, j=1}^{2} \int_{R_{i}^{j}} \phi\left(\mathbf{e}_{\mathbf{1}}\right) \mathrm{d} \mathcal{H}^{1},
\end{aligned}
$$

where $\gamma_{j}:=R_{1}^{j} \cup K^{j} \cup R_{2}^{j}$ is the curve connecting $(-1,0)$ to $(1,0)$. By the (anisotropic) minimality of segments [30, Lemma 6.2],

$$
\int_{\gamma_{j}} \phi\left(v_{\gamma_{j}}\right) \mathrm{d} \mathcal{H}^{1} \geqq \int_{I_{1}} \phi\left(\mathbf{e}_{2}\right) \mathrm{d} \mathcal{H}^{1}
$$

Moreover, since $\mathcal{H}^{1}\left(R_{i}^{j}\right) \leqq \frac{\delta}{16 c_{2} m_{o}}$ for any $i, j=1,2$, by (4.11), (4.12) and (2.4) we obtain

$$
\alpha_{k} \geqq 2 \int_{I_{1}} \phi\left(\mathbf{e}_{2}\right) \mathrm{d} \mathcal{H}^{1}-\frac{4 c_{2} \delta}{16 c_{2} m_{o}}=2 \int_{I_{1}} \phi\left(\mathbf{e}_{2}\right) \mathrm{d} \mathcal{H}^{1}-\frac{\delta}{4 m_{o}},
$$

which implies (4.10).

Step 2. Assume now that every connected component of $U_{1} \cap \partial E_{k}$ intersects at most one of $\left\{x_{1}=1\right\}$ and $\left\{x_{1}=-1\right\}$. In this case, let $K^{1}, \ldots, K^{m_{k}}$ stand for the connected components of $\partial E_{k}$ lying strictly inside of $U_{1}$ (that is, not intersecting $\left\{x_{1}= \pm 1\right\}$ ); by (b), $m_{k} \leqq m_{o}$. Since $\alpha_{k}<\infty$, the connected components $\left\{L^{i}\right\}$ of $\overline{U_{1}} \cap \partial E_{k}$ intersecting $\left\{x_{1}= \pm 1\right\}$ is at most countable. If $\left\{L^{i}: L^{i} \cap\left\{x_{1}=1\right\} \neq\right.$ $\emptyset\}=\emptyset$, we set $K^{m_{k}+1}=\emptyset$ otherwise let $K^{m_{k}+1}$ be such that $\pi\left(K^{m_{k}+1}\right)$ contains all $\pi\left(L^{i}\right)$ with $L^{i} \cap\left\{x_{1}=1\right\} \neq \emptyset$, where $\pi$ is given by (4.5). Analogously, we define $K^{m_{k}+2} \in\left\{L^{i}: L^{i} \cap\left\{x_{1}=-1\right\} \neq \emptyset\right\} \cup\{\emptyset\}$. By the connectedness of $K^{j}$, for each $j=1, \ldots, m_{k}+2, \pi\left(K^{j}\right)$ (if non-empty) is a segment $\left[a_{k}^{i}, b_{k}^{i}\right] \times\{0\}$. Then assumption (a) and the bound $m_{k} \leqq m_{o}$ imply that

$$
\lim _{k \rightarrow \infty} \mathcal{H}^{1}\left(I_{1} \backslash \bigcup_{j=1}^{m_{k}+2} \pi\left(K^{j}\right)\right)=0 .
$$


Hence there exists $k_{2, \delta}>k_{1, \delta}$ such that

$$
\mathcal{H}^{1}\left(I_{1} \backslash \bigcup_{i=1}^{m_{k}+2} \pi\left(K^{j}\right)\right)<\frac{\delta}{8 c_{2} m_{o}}
$$

for any $k>k_{2, \delta}$. Then repeating the proof of (4.13) with $K^{j}$ in $\left(a_{j}, b_{j}\right) \times(-1,1)$, for every $j=1, \ldots, m_{k}+2$ we find

$\int_{K^{j} \cap \partial^{*} E_{k}} \phi\left(v_{E_{k}}\right) \mathrm{d} \mathcal{H}^{1}+2 \int_{K^{j} \cap\left(E_{k}^{(1)} \cup E_{k}^{(0)}\right) \cap \partial E_{k}} \phi\left(v_{E_{k}}\right) \mathrm{d} \mathcal{H}^{1} \geqq 2 \int_{\pi\left(K^{j}\right)} \phi\left(\mathbf{e}_{2}\right) \mathrm{d} \mathcal{H}^{1}-\frac{\delta}{4 m_{o}}$.

Therefore, by (4.14) and (2.4),

$$
\begin{aligned}
\alpha_{k} & \geqq \sum_{j=1}^{m_{k}+2} \int_{K^{j} \cap \partial^{*} E_{k}} \phi\left(v_{E_{k}}\right) \mathrm{d} \mathcal{H}^{1}+2 \int_{K^{j} \cap\left(E_{k}^{(1)} \cup E_{k}^{(0)}\right) \cap \partial E_{k}} \phi\left(v_{E_{k}}\right) \mathrm{d} \mathcal{H}^{1} \\
& \geqq \sum_{j=1}^{m_{k}+2}\left(2 \int_{\pi\left(K^{j}\right)} \phi\left(\mathbf{e}_{2}\right) \mathrm{d} \mathcal{H}^{1}-\frac{\delta}{4 m_{o}}\right) \geqq 2 \int_{\bigcup \pi\left(K^{j}\right)} \phi\left(\mathbf{e}_{2}\right) \mathrm{d} \mathcal{H}^{1}-\frac{\left(m_{k}+2\right) \delta}{4 m_{o}} \\
& \geqq 2 \int_{I_{1}} \phi\left(\mathbf{e}_{2}\right) \mathrm{d} \mathcal{H}^{1}-2 c_{2} \frac{\delta}{8 c_{2} m_{o}}-\frac{\left(m_{o}+2\right) \delta}{4 m_{o}} \\
& =2 \int_{I_{1}} \phi\left(\mathbf{e}_{2}\right) \mathrm{d} \mathcal{H}^{1}-\frac{\left(m_{o}+3\right) \delta}{4 m_{o}} .
\end{aligned}
$$

Since $m_{o} \geqq 1$, this implies (4.10).

Lemma 4.5. (Creation of internal crack energy)

Let $\phi$ be as in Lemma 4.4 and let $\left\{E_{k}\right\}$ be a sequence of subsets of $U_{1}$ such that

(a) $U_{1} \backslash E_{k} \stackrel{\mathcal{K}}{\rightarrow} I_{1}=[-1,1] \times\{0\}$ as $k \rightarrow \infty$;

(b) there exists $m_{o} \in \mathbb{N}_{0}$ such that the number of connected components of each $\partial E_{k}$ lying strictly inside $U_{1}$ does not exceed $m_{o}$.

Then for every $\delta \in(0,1)$ there exists $k_{\delta}>1$ such that for any $k>k_{\delta}$,

$\int_{U_{1} \cap \partial^{*} E_{k}} \phi\left(v_{E_{k}}\right) d \mathcal{H}^{1}+2 \int_{U_{1} \cap\left(E_{k}^{(0)} \cup E_{k}^{(1)}\right) \cap \partial E_{k}} \phi\left(v_{E_{k}}\right) d \mathcal{H}^{1} \geqq 2 \int_{I_{1}} \phi\left(\mathbf{e}_{2}\right) d \mathcal{H}^{1}-\delta$.

Proof. The assertion follows from applying Lemma 4.5 to $U_{1} \backslash E_{k}$.

The following result extends the lower semicontinuity result of [15, Theorem 1.1] to the anisotropic case.

Proposition 4.6. Let $D \subset \mathbb{R}^{d}$ be a bounded open set and let $\phi \in C(\bar{D} \times$ $\mathbb{R}^{d} ;[0,+\infty)$ be a Finsler norm in $\mathbb{R}^{d}, d \geqq 2$, satisfying

$$
c_{1} \leqq \phi(x, v) \leqq c_{2}, \quad(x, v) \in \bar{D} \times \mathbb{S}^{d-1},
$$


for some $c_{2} \geqq c_{1}>0$. Consider $\left\{w_{h}\right\} \subset G S B D^{2}\left(D ; \mathbb{R}^{d}\right)$ such that

$$
\sup _{k \geqq 1} \int_{D}\left|e\left(w_{h}\right)\right|^{2} d x+\mathcal{H}^{1}\left(J_{w_{h}}\right) \leqq M
$$

for some $M>0$ and the set

$$
E:=\left\{x \in D: \liminf _{h \rightarrow \infty}\left|w_{h}(x)\right|=+\infty\right\}
$$

has finite perimeter. Suppose that $w_{h} \rightarrow w$ a.e. in $D \backslash E$ as $h \rightarrow \infty$ (so that by [15, Theorem 1.1] $\left.w \in G S B D^{2}\left(D \backslash E ; \mathbb{R}^{d}\right)\right)$. Then

$$
\int_{J_{w} \cup \partial^{*} E} \phi\left(x, v_{J_{w} \cup \partial E}\right) d \mathcal{H}^{d-1} \leqq \liminf _{h \rightarrow \infty} \int_{J_{w_{h}}} \phi\left(x, v_{J_{w_{h}}}\right) d \mathcal{H}^{d-1} .
$$

Proof. We divide the proof into two steps.

Step 1. First we prove the (4.17) assuming that $\phi$ is independent on $x \in D$, i.e., $\phi(v)=\phi(x, v)$ for any $x \in \bar{D} \times \mathbb{R}^{d}$.

Let $W=\left\{\phi^{\circ} \leqq 1\right\}$ be the Wulff shape of $\phi$, i.e, the unit ball for the dual norm

$$
\phi^{\circ}(\xi)=\max _{\phi(\eta)=1}|\xi \cdot \eta|
$$

Note that $\phi^{\circ \circ}=\phi$ and by (4.15),

$$
\frac{1}{c_{2}}|\xi| \leqq \phi^{\circ}(\xi) \leqq \frac{1}{c_{1}}|\xi|
$$

for any $\xi \in \mathbb{R}^{2}$. Let $\left\{\xi_{n}\right\} \subset \partial W$ be a countable dense set. Then since

$$
\phi(v)=\sup _{n \in \mathbb{N}}\left|\xi_{n} \cdot v\right|,
$$

from [25, Lemma 6] it follows that for every bounded open set $G$ and $u \in$ $G S B D^{2}\left(G ; \mathbb{R}^{d}\right)$,

$$
\int_{G \cap J_{u}} \phi\left(v_{J_{u}}\right) \mathrm{d} \mathcal{H}^{d-1}=\sup \sum_{n=1}^{N} \int_{F_{n} \cap J_{u}}\left|\xi_{n} \cdot v_{J_{u}}\right| \mathrm{d} \mathcal{H}^{d-1},
$$

where sup is taken over finite disjoint open sets $\left\{F_{n}\right\}_{n=1}^{N}$ whose closures are contained in $G$.

Now we prove (4.17). Under the notation of [7,15], for any $\epsilon \in(0,1)$, open set $F \subset D$ with $\bar{F} \subset D$ and for $\mathcal{H}^{1}$-a.e. $\xi \in \partial W$ we have

$$
\begin{aligned}
|\xi| \int_{\Pi_{\xi}}\left[\mathcal { H } ^ { 0 } \left(F_{y}^{\xi}\right.\right. & \left.\left.\cap J_{\widehat{w}_{y}^{\xi}} \cap(F \backslash E)_{y}^{\xi}\right)+\mathcal{H}^{0}\left(F_{y}^{\xi} \cap \partial E_{y}^{\xi}\right)\right] \mathrm{d} \mathcal{H}^{d-1} \\
& \leqq|\xi| \liminf _{h \rightarrow \infty} \int_{\Pi_{\xi}}\left[\mathcal{H}^{0}\left(F_{y}^{\xi} \cap J_{\left(\widehat{w}_{h}\right)_{y}^{\xi}}\right)+c_{2}^{-1} \epsilon f_{y}^{\xi}\left(w_{h}\right)\right] \mathrm{d} \mathcal{H}^{d-1}
\end{aligned}
$$


where $\Pi_{\xi}:=\left\{y \in \mathbb{R}^{d}: y \cdot \xi=0\right\}$, is the hyperplane passing through the origin and orthogonal to $\xi$, given $y \in \mathbb{R}^{d}, F_{y}^{\xi}:=\{t \in \mathbb{R}: y+t \xi \in F\}$ is the section of the straight line passing through $y \in \mathbb{R}^{d}$ and parallel to $\xi$, given $u: F \rightarrow \mathbb{R}^{d}$ and $y \in \mathbb{R}^{d}, \widehat{u}_{y}^{\xi}: F_{y}^{\xi} \rightarrow \mathbb{R}$ is defined as $\widehat{u}_{y}^{\xi}(t):=u(y+t \xi) \cdot \xi$, and

$$
f_{y}^{\xi}\left(w_{h}\right)=I_{y}^{\xi}\left(w_{h}\right)+I I_{y}^{\xi}\left(w_{h}\right)
$$

with

$$
\int_{\Pi_{\xi}} I_{y}^{\xi}\left(w_{h}\right) \mathrm{d} \mathcal{H}^{1} \leqq \int_{F}\left|e\left(w_{h}(x)\right)\right|^{2} \mathrm{~d} x, \quad h \geqq 1,
$$

and

$$
\int_{\Pi_{\xi}} I I_{y}^{\xi}\left(w_{h}\right) \mathrm{d} \mathcal{H}^{1} \leqq\left|D_{\xi}\left(\tau\left(w_{h} \cdot \xi\right)\right)\right|(F), \quad h \geqq 1,
$$

for $\tau(t):=\tanh (t)$ (see [15, Eq.s 3.10 and 3.11] applied with $F$ in place of $\Omega$ ).

By [2, Theorem 4.10] and (4.18), (4.20) can be rewritten as

$$
\begin{aligned}
& \int_{F \cap\left(J_{w} \cup \partial^{*} E\right)}\left|v_{J_{w} \cup \partial E} \cdot \xi\right| \mathrm{d} \mathcal{H}^{d-1} \leqq \liminf _{h \rightarrow \infty} \int_{F \cap J_{w_{h}}}\left|v_{J_{w_{h}}} \cdot \xi\right| \mathrm{d} \mathcal{H}^{d-1} \\
& +\epsilon \int_{F}\left|e\left(w_{h}(x)\right)\right|^{2} \mathrm{~d} x+\epsilon\left|D_{\xi}\left(\tau\left(w_{h} \cdot \xi\right)\right)\right|(F) .
\end{aligned}
$$

Fix any finite family $\left\{F_{n}\right\}_{n=1}^{N}$ of pairwise disjoint open sets whose closures are contained in $D$. Since (4.22) holds for $\mathcal{H}^{1}$-a.e. $\xi \in \partial W$, we can extract a countable dense set $\left\{\xi_{n}\right\} \subset \partial W$ satisfying (4.22) with $\xi=\xi_{i}$ and $F=F_{j}$ for all $i, j$. Now taking $F=F_{n}$ and $\xi=\xi_{n}$ in (4.22) and summing over $n=1, \ldots, N$, we get

$$
\begin{aligned}
& \sum_{n=1}^{N} \int_{F_{n} \cap\left(J_{w} \cup \partial^{*} E\right)}\left|v_{J_{w} \cup \partial E} \cdot \xi_{n}\right| \mathrm{d} \mathcal{H}^{d-1} \leqq \liminf _{h \rightarrow \infty} \sum_{n=1}^{N} \int_{F_{n} \cap J_{w_{h}}}\left|v_{J_{w_{h}}} \cdot \xi_{n}\right| \mathrm{d} \mathcal{H}^{d-1} \\
& +\epsilon \int_{\bigcup_{n=1}^{N} F_{n}}\left|e\left(w_{h}(x)\right)\right|^{2} \mathrm{~d} x+\epsilon\left|D_{\xi_{n}}\left(\tau\left(w_{h} \cdot \xi_{n}\right)\right)\right|\left(\bigcup_{n=1}^{N} F_{n}\right) .
\end{aligned}
$$

Recall that by (4.19),

$$
\sum_{n=1}^{N} \int_{F_{n} \cap J_{w_{h}}}\left|v_{J_{w_{h}}} \cdot \xi_{n}\right| \mathrm{d} \mathcal{H}^{1} \leqq \int_{J_{w_{h}}} \phi\left(v_{J_{w_{h}}}\right) \mathcal{H}^{d-1}
$$

and by (4.16),

$$
\int_{n=1}^{N}\left|e\left(w_{h}(x)\right)\right|^{2} \mathrm{~d} x \leqq \int_{D}\left|e\left(w_{h}(x)\right)\right|^{2} \mathrm{~d} x \leqq M
$$


and

$$
\left|D_{\xi_{n}}\left(\tau\left(w_{h} \cdot \xi_{n}\right)\right)\right|\left(\bigcup_{n=1}^{N} F_{n}\right) \leqq\left|D_{\xi_{n}}\left(\tau\left(w_{h} \cdot \xi_{n}\right)\right)\right|(D) \leqq M
$$

for any $h \geqq 1$. Therefore,

$$
\sum_{n=1}^{N} \int_{F_{n} \cap\left(J_{w} \cup \partial^{*} E\right)}\left|v_{J_{w} \cup \partial E} \cdot \xi_{n}\right| \mathrm{d} \mathcal{H}^{d-1} \leqq 2 M \epsilon+\liminf _{h \rightarrow \infty} \int_{J_{w_{h}}} \phi\left(v_{J_{w_{h}}}\right) \mathcal{H}^{d-1} .
$$

Now taking sup over $\left\{F_{n}\right\}$ and letting $\epsilon \rightarrow 0$ we obtain (4.17).

Step 2. Now we prove (4.17) in general case. Without loss of generality we suppose that the liminf in (4.17) is a finite limit. Consider the sequence $\left\{\mu_{h}\right\}_{h \geqq 0}$ of positive Radon measures in $D$ defined at Borel subsets of $B \subseteq D$ as

$$
\mu_{h}(B)=\int_{B \cap J_{w_{h}}} \phi\left(x, v_{J_{w_{h}}}\right) \mathrm{d} \mathcal{H}^{d-1}, \quad h \geqq 1,
$$

and

$$
\mu_{0}(B)=\int_{B \cap J_{w}} \phi\left(x, v_{J_{w}}\right) \mathrm{d} \mathcal{H}^{d-1} .
$$

Since $\sup _{h} \mu_{h}(D)<\infty$, by compactness, there exist a positive Radon measure $\mu$ and a not relbelled subsequence $\left\{\mu_{h}\right\}_{h \geq 1}$ such that $\mu_{h} \rightarrow * \mu$ as $h \rightarrow \infty$. We prove that

$$
\mu \geqq \mu_{0},
$$

in particular from $\mu(D) \geqq \mu_{0}(D)$ (4.17) follows. Since $\mu_{0}$ is absolutely continuous with respect to $\mathcal{H}^{d-1}\left\llcorner J_{w}\right.$, to prove (4.23) we need only to show

$$
\frac{\mathrm{d} \mu}{\mathrm{d} \mathcal{H}^{d-1}\left\llcorner J_{w}\right.}(x) \geqq \phi\left(x, v_{J_{w}}\right) \quad \text { for } \mathcal{H}^{d-1}-\text { almost everywhere } x \in J_{w} \text {. }
$$

For this aim fix $\epsilon \in\left(0, c_{1}\right)$. By the uniform continuity of $\phi$, there exists $r_{\epsilon}>0$ such that

$$
|\phi(x, v)-\phi(y, v)| \leqq \epsilon
$$

for any $v \in \mathbb{S}^{d-1}$ and $x, y \in D$ with $|x-y|<r_{\epsilon}$. In particular, given $x \in J_{w}$ and for a.e. $r \in\left(0, r_{\epsilon}\right)$,

$$
\begin{aligned}
\mu\left(B_{r}\left(x_{0}\right)\right) & =\lim _{h \rightarrow \infty} \mu_{h}\left(B_{r}\left(x_{0}\right)\right) \\
\geqq & \liminf _{h \rightarrow \infty} \int_{B_{r}\left(x_{0}\right) \cap J_{w_{h}}} \phi\left(x_{0}, v_{J_{w_{h}}}\right) \mathrm{d} \mathcal{H}^{d-1}-\epsilon \limsup _{h \rightarrow \infty} \mathcal{H}^{d-1}\left(B_{r}\left(x_{0}\right) \cap J_{w_{h}}\right),
\end{aligned}
$$

where in the equality we use the weak* convergence of $\left\{\mu_{h}\right\}$ and in the inequality (4.25) with $y=x_{0}$ and $x \in B_{r}\left(x_{0}\right) \cap J_{w_{h}}$ By Proposition 4.6 applied with $\phi\left(x_{0}, \cdot\right)$, 


$$
\begin{aligned}
\liminf _{h \rightarrow \infty} \int_{B_{r}\left(x_{0}\right) \cap J_{w_{h}}} \phi\left(x_{0}, v_{J_{w_{h}}}\right) \mathrm{d} \mathcal{H}^{d-1} & \geqq \int_{B_{r}\left(x_{0}\right) \cap J_{w}} \phi\left(x_{0}, v_{J_{w}}\right) \mathrm{d} \mathcal{H}^{d-1} \\
& \geqq \mu_{0}\left(B_{r}\left(x_{0}\right)\right)-\epsilon \mathcal{H}^{d-1}\left(B_{r}\left(x_{0}\right) \cap J_{w}\right),
\end{aligned}
$$

where in the second equality we again used (4.25). Moreover, by (4.15),

$$
\limsup _{h \rightarrow \infty} \mathcal{H}^{d-1}\left(B_{r}\left(x_{0}\right) \cap J_{w_{h}}\right) \leqq \frac{1}{c_{1}} \limsup _{h \rightarrow \infty} \mu_{h}\left(B_{r}\left(x_{0}\right)\right)=\frac{\mu\left(B_{r}\left(x_{0}\right)\right)}{c_{1}}
$$

and

$$
\mathcal{H}^{d-1}\left(B_{r}\left(x_{0}\right) \cap J_{w}\right) \leqq \frac{\mu_{0}\left(B_{r}\left(x_{0}\right)\right)}{c_{1}}
$$

thus,

$$
\mu\left(B_{r}\left(x_{0}\right)\right) \geqq \frac{c_{1}-\epsilon}{c_{1}+\epsilon} \mu_{0}\left(B_{r}\left(x_{0}\right)\right) .
$$

Since $\epsilon$ and $r \in\left(0, r_{\epsilon}\right)$ are arbitrary, (4.24) follows from the Besicovitch Derivation Theorem.

\section{Lemma 4.7. (Creation of delamination energy)}

Let $\phi$ be as in Lemma 4.4 and suppose that $\Omega_{k} \subset U_{4}$ is a sequence of Lipschitz sets, $E_{k} \subset \Omega_{k}, J_{E_{k}} \in \mathcal{J}_{E_{k}}$, and $g_{0}, g_{1} \in[0,+\infty), u_{k} \in G S B D^{2}\left(U_{4} ; \mathbb{R}^{2}\right)$ and $u^{ \pm} \in \mathbb{R}^{2}$ with $u^{+} \neq u^{-}$are such that

(a) $\operatorname{sdist}\left(\cdot, U_{4} \cap \partial \Omega_{k}\right) \rightarrow \operatorname{sdist}\left(\cdot, \partial U_{4}^{+}\right)$uniformly in $U_{3 / 2}$;

(b) $\Sigma_{k}:=U_{4} \cap \partial \Omega_{k}$ is a graph of a Lipschitz function $l_{k}: I_{4} \rightarrow \mathbb{R}$ such that $l_{k}(0)=0$ and $\left|l_{k}^{\prime}\right| \leqq \frac{1}{k}$;

(c) $\operatorname{sdist}\left(\cdot, U_{4} \cap \partial E_{k}\right) \rightarrow \operatorname{sdist}\left(\cdot, \partial U_{4}^{+}\right)$uniformly in $U_{3 / 2}$;

(d) there exists $m_{k} \in \mathbb{N}_{0}$ such that the number of connected components of each $\partial E_{k}$ lying strictly inside $\Omega_{k}$ does not exceed $m_{k}$;

(e) $\left|g_{1}-g_{0}\right| \leqq \phi\left(\mathbf{e}_{2}\right)$;

(f) $J_{u_{k}} \subset\left(\Omega_{k} \cap \partial E_{k}\right) \cup J_{E_{k}} \cup L_{k}$, where $L_{k} \subset \Sigma_{k}$ is a relatively open subset of $\Sigma_{k}$ with $\mathcal{H}^{1}\left(L_{k}\right)<1 / k$

(g) $\sup \int_{U_{4}}\left|e\left(u_{k}\right)\right|^{2} d x<\infty$; $k \geqq 1$

(h) $u_{k} \rightarrow u^{+}$a.e. in $U_{1}^{+}$and $u_{k} \rightarrow u^{-}$a.e. in $U_{1} \backslash \overline{U_{1}^{+}}$.

Then for every $\delta \in(0,1)$ there exists $k_{\delta}>1$ for which

$$
\begin{gathered}
\int_{U_{1} \cap \Omega_{k} \cap \partial^{*} E_{k}} \phi\left(v_{E_{k}}\right) d \mathcal{H}^{1}+2 \int_{U_{1} \cap \Omega_{k} \cap\left(E_{k}^{(1)} \cup E_{k}^{(0)}\right) \cap \partial E_{k}} \phi\left(v_{E_{k}}\right) d \mathcal{H}^{1} \\
+\int_{U_{1} \cap \Sigma_{k} \backslash \partial E_{k}} g_{0} d \mathcal{H}^{1}+\int_{U_{1} \cap \Sigma_{k} \cap E_{k}^{(0)} \cap \partial E_{k}}\left(\phi\left(v_{\Sigma}\right)+g_{1}\right) d \mathcal{H}^{1}
\end{gathered}
$$




$$
\begin{aligned}
& +\int_{U_{1} \cap \Sigma_{k} \cap \partial^{*} E_{k} \backslash J_{E_{k}}} g_{1} d \mathcal{H}^{1}+\int_{U_{1} \cap J_{E_{k}}}\left(\phi\left(v_{\Sigma}\right)+g_{0}\right) d \mathcal{H}^{1} \\
\geqq & \int_{I_{1}}\left(\phi\left(\mathbf{e}_{2}\right)+g_{0}\right) d \mathcal{H}^{1}-\delta
\end{aligned}
$$

for any $k>k_{\delta}$.

Proof. Denote the left-hand-side of (4.26) by $\alpha_{k}$. We suppose that $\sup _{k}\left|\alpha_{k}\right|<\infty$ so that by (4.9)

$$
\sup _{k} \mathcal{H}^{1}\left(\partial E_{k}\right) \leqq M
$$

for some $M>0$. Moreover, passing to a not relabelled subsequence if necessary, we assume that

$$
\liminf _{k \rightarrow \infty} \alpha_{k}=\lim _{k \rightarrow \infty} \alpha_{k}
$$

By assumption (b), $\Sigma_{k}$ is "very close" $I_{2}$, hence, by the area formula [3, Theorem 2.91] for any $K_{k} \subset \Sigma_{k}$ one has

$$
\begin{aligned}
& \underset{k \rightarrow \infty}{\limsup }\left|\int_{\pi\left(K_{k}\right)} \phi\left(\mathbf{e}_{2}\right) \mathrm{d} \mathcal{H}^{1}-\int_{K_{k}} \phi\left(v_{\Sigma_{k}}\right) \mathrm{d} \mathcal{H}^{1}\right| \\
& \leqq \limsup _{k \rightarrow \infty} \int_{\pi\left(K_{k}\right)}\left|\phi\left(\mathbf{e}_{2}\right)-\phi\left(l_{k}^{\prime}, 1\right)\right| \mathrm{d} \mathcal{H}^{1} \leqq \limsup _{k \rightarrow \infty} \int_{\pi\left(K_{k}\right)}\left|\phi\left(\mathbf{e}_{2}-\left(l_{k}^{\prime}, 1\right)\right)\right| \mathrm{d} \mathcal{H}^{1} \\
& =\limsup _{k \rightarrow \infty} \int_{\pi\left(K_{k}\right)} \phi(0,1)\left|l_{k}^{\prime}\right| \mathrm{d} \mathcal{H}^{1}=0,
\end{aligned}
$$

where in the last inequality and in the first equality we used that $\phi$ is a norm, and the last equality follows from $\left|l_{k}^{\prime}\right| \leqq \frac{1}{k}$. Hence,

$$
\lim _{k \rightarrow \infty}\left|\int_{\pi\left(K_{k}\right)} \phi\left(\mathbf{e}_{2}\right) \mathrm{d} \mathcal{H}^{1}-\int_{K_{k}} \phi\left(v_{\Sigma_{k}}\right) \mathrm{d} \mathcal{H}^{1}\right|=0 .
$$

We divide the proof into two steps.

Step 1. For shortness, let $J_{k}:=J_{E_{k}}$ and $C_{k}:=\Sigma_{k} \backslash \overline{\partial^{*} E_{k}}$. We claim that for any $\delta \in(0,1)$ there exists $k_{\delta}^{1}>0$ such that for any $k>k_{\delta}^{1}$,

$$
\begin{gathered}
\int_{U_{1} \cap \Omega_{k} \cap \partial^{*} E_{k}} \phi\left(v_{E_{k}}\right) \mathrm{d} \mathcal{H}^{1}+2 \int_{U_{1} \cap \Omega_{k} \cap\left(E_{k}^{(1)} \cup E_{k}^{(0)}\right) \cap \partial E_{k}} \phi\left(v_{E_{k}}\right) \mathrm{d} \mathcal{H}^{1} \\
\geqq 2 \int_{U_{1} \cap \Sigma_{k} \backslash\left(J_{k} \cup C_{k}\right)} \phi\left(v_{\Sigma_{k}}\right) \mathrm{d} \mathcal{H}^{1}+\int_{U_{1} \cap C_{k}} \phi\left(v_{\Sigma_{k}}\right) \mathrm{d} \mathcal{H}^{1}-\delta .
\end{gathered}
$$

Indeed, by adding to both sides of (4.29) the quantity $2 \int_{U_{1} \cap J_{k}} \phi\left(v_{\Sigma_{k}}\right) \mathrm{d} \mathcal{H}^{1}+$ $\int_{U_{1} \cap C_{k}} \phi\left(v_{\Sigma_{k}}\right) \mathrm{d} \mathcal{H}^{1},(4.29)$ is equivalent to 


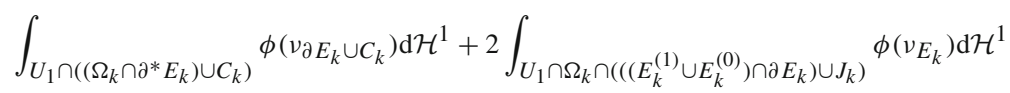

$$
\begin{aligned}
& \geqq 2 \int_{U_{1} \cap \Sigma_{k}} \phi\left(v_{\Sigma_{k}}\right) \mathrm{d} \mathcal{H}^{1}-\delta,
\end{aligned}
$$

and hence, we will prove (4.30).

Note that since $J_{k} \subset \Sigma_{k}$ is $\mathcal{H}^{1}$-rectifiable, given $\delta \in(0,1)$ there exists a finite union $R_{k}$ of intervals of $\Sigma_{k}$ such that

$$
J_{k} \cup L_{k} \subset R_{k} \text { and } \quad \mathcal{H}^{1}\left(R_{k} \backslash\left(J_{k} \cup L_{k}\right)\right)<\frac{\delta}{5 c_{2}},
$$

where $c_{2}>0$ is given in (4.9). Possibly slightly modifying $u_{k}$ around the (approximate) continuity points of $R_{k}$ and around the boundary of the voids $U_{2} \backslash E_{k}$ we assume that $J_{k}:=R_{k}, L_{k}=\emptyset$ and $J_{u_{k}}=\left(\Omega_{k} \cap \partial E_{k}\right) \cup C_{k} \cup J_{k}$ (up to a $\mathcal{H}^{1}$ negligible set).

Let $K_{k}=U_{1} \cap\left(\overline{\Omega_{k} \cap \partial E_{k}} \cup \overline{J_{k} \cup C_{k}}\right)$. By relative openness of $C_{k}=\Sigma_{k} \backslash \overline{\partial^{*} E_{k}}$ and $J_{k}$ in $\Sigma_{k}$ and assumption (d), $K_{k}$ is a union $\bigcup_{j} K_{k}^{j}$ of at most countably many pairwise disjoint connected rectifiable sets $K_{k}^{j}$ relatively closed in $U_{1}$.

Let $c o\left(K_{k}^{j}\right)$ denote the closed convex hull of $K_{k}^{j}$. Observe that if $K_{k}^{j}$ is not a segment, then the interior of $c o\left(K_{k}^{j}\right)$ is non-empty and

$$
\begin{aligned}
& \int_{K_{k}^{j} \cap\left(\partial^{*} E_{k} \cup C_{k}\right)} \phi\left(v_{K_{k}}\right) \mathrm{d} \mathcal{H}^{1}+2 \int_{K_{k}^{j} \cap\left(\left(\left(E_{k}^{(1)} \cup E_{k}^{(0)}\right) \cap \partial E_{k}\right) \cup J_{k}\right)} \phi\left(v_{K_{k}}\right) \mathrm{d} \mathcal{H}^{1} \\
& \quad \geqq \int_{\partial \operatorname{co}\left(K_{k}^{j}\right)} \phi\left(v_{c o\left(K_{k}^{j}\right)}\right) \mathrm{d} \mathcal{H}^{1} .
\end{aligned}
$$

Now we define the minimal union of disjoint closed convex sets containing $K_{k}$ as follows. For every $k \geqq 1$ let us define the sequences $\left\{D_{i}^{k}\right\}_{i}$ of pairwise disjoint subsets of $\mathbb{N}$ and $\left\{V_{i}^{k}\right\}_{i}$ of pairwise disjoint closed convex subsets of $\overline{U_{1}}$ as follows. Let $D_{0}^{k}:=\{1\}$ and $V_{0}^{k}:=\emptyset$. Suppose that for some $i \geqq 1$ the sets $D_{0}^{k}, \ldots, D_{i-1}^{k}$ and $V_{0}^{k}, \ldots, V_{i-1}^{k}$ are defined and let $j_{o}$ be the smallest element of $\mathbb{N} \backslash \bigcup_{j=0}^{i-1} D_{j}^{k}$. Define

$$
D_{i}^{k}:=\left\{h \in \mathbb{N} \backslash \bigcup_{j=0}^{i-1} D_{j}^{k}: \operatorname{co}\left(K_{k}^{j_{o}}\right) \cap \operatorname{co}\left(K_{k}^{h}\right) \neq \emptyset\right\}
$$

and

$$
V_{i}^{k}:=\operatorname{co}\left(\cup_{h \in D_{i}^{k}} K_{k}^{h}\right)
$$


Note that $j_{o} \in D_{i}^{k}$. As in (4.32) we observe that

$$
\begin{aligned}
& \sum_{h \in D_{i}^{k}} \int_{K_{k}^{h} \cap\left(\partial^{*} E_{k} \cup C_{k}\right)} \phi\left(v_{K_{k}}\right) \mathrm{d} \mathcal{H}^{1}+2 \int_{K_{k}^{h} \cap\left(\left(\left(E_{k}^{(1)} \cup E_{k}^{(0)}\right) \cap \partial E_{k}\right) \cup J_{k}\right)} \phi\left(v_{K_{k}}\right) \mathrm{d} \mathcal{H}^{1} \\
& \geqq \int_{\partial V_{i}^{k}} \phi\left(v_{V_{i}^{k}}\right) \mathrm{d} \mathcal{H}^{1} .
\end{aligned}
$$

Then $K_{k} \subset \bigcup_{i} V_{i}^{k}$ and by (4.33)

$$
\begin{aligned}
& \int_{K \cap\left(\partial^{*} E_{k} \cup C_{k}\right)} \phi\left(v_{K_{k}}\right) \mathrm{d} \mathcal{H}^{1}+2 \int_{K_{k} \cap\left(\left(\left(E_{k}^{(1)} \cup E_{k}^{(0)}\right) \cap \partial E_{k}\right) \cup J_{k}\right)} \phi\left(v_{K_{k}}\right) \mathrm{d} \mathcal{H}^{1} \\
& \geqq \sum_{i \notin T} \int_{\partial V_{i}^{k}} \phi\left(v_{V_{i}^{k}}\right) \mathrm{d} \mathcal{H}^{1}+2 \sum_{i \in T} \int_{\partial V_{i}^{k}} \phi\left(v_{V_{i}^{k}}\right) \mathrm{d} \mathcal{H}^{1},
\end{aligned}
$$

where $T$ is the set of all indices $i$ for which $V_{i}^{k}$ is a line segment. For every $i \in T$ we replace the segment $V_{i}^{k}$ with a closed rectangle $Q_{i}$ containing $V_{i}^{k}$ and not intersecting any $V_{j}^{k}, j \neq i$, such that

$$
2 \int_{V_{i}^{k}} \phi\left(v_{V_{i}^{k}}\right) \mathrm{d} \mathcal{H}^{1} \geqq \int_{\partial Q_{i}} \phi\left(v_{Q_{i}}\right) \mathrm{d} \mathcal{H}^{1}-\frac{\delta}{10 \cdot 2^{i}}
$$

Therefore, redefining $V_{i}^{k}:=Q_{i}$ we obtain

$$
\begin{aligned}
& \int_{K_{k} \cap\left(\partial^{*} E_{k} \cup C_{k}\right)} \phi\left(v_{K_{k}}\right) \mathrm{d} \mathcal{H}^{1}+2 \int_{K_{k} \cap\left(\left(\left(E_{k}^{(1)} \cup E_{k}^{(0)}\right) \cap \partial E_{k}\right) \cup J_{k}\right)} \phi\left(v_{K_{k}}\right) \mathrm{d} \mathcal{H}^{1} \\
& \geqq \sum_{i} \int_{\partial V_{i}^{k}} \phi\left(v_{V_{i}^{k}}\right) \mathrm{d} \mathcal{H}^{1}-\frac{\delta}{5} .
\end{aligned}
$$

Note that $U_{1} \backslash \bigcup_{i} V_{i}^{k}$ is a Lipschitz open set and $J_{u_{k}} \cap\left(U_{1} \backslash \bigcup_{i} V_{i}^{k}\right)=\varnothing$, and hence, by the Poincaré-Korn inequality, $u_{k} \in H^{1}\left(U_{1} \backslash \bigcup_{i} V_{i}^{k}\right)$. Moreover, (4.27), (4.9) and (4.34) imply $c_{1} \sum_{i} \mathcal{H}^{1}\left(\partial V_{i}^{k}\right)<M+1$, thus, there exists $\eta \in \mathbb{R}^{2}$ such that the set

$$
\left\{x \in U_{1} \cap \bigcup_{i} \partial V_{i}^{k}: \text { trace of }\left.u_{k}\right|_{U_{1} \backslash \cup V_{i}^{k}} \text { is equal to } \eta\right\}
$$

is $\mathcal{H}^{1}$-negligible (see [46, Proposition 2.6]). Therefore, $v_{k}:=u_{k} \chi_{U_{1} \backslash \cup V_{i}^{k}}+\eta \chi_{\cup V_{i}^{k}}$ belongs to $G S B D^{2}\left(U_{1} ; \mathbb{R}^{2}\right), J_{v_{k}}=U_{1} \cap \cup_{i} \partial V_{i}^{k}$. By assumptions (a), (c) and (h), $v_{k} \rightarrow v:=u^{+} \chi_{U_{1}^{+}}+u^{-} \chi_{U_{1} \backslash U_{1}^{+}}$, and by assumption (g) and inequalities (4.9), and (4.27),

$$
\sup _{k} \int_{U_{1}}\left|e\left(v_{k}\right)\right|^{2} \mathrm{~d} x+\mathcal{H}^{1}\left(J_{v_{k}}\right) \leqq \sup _{k} \int_{U_{1}}\left|e\left(u_{k}\right)\right|^{2} \mathrm{~d} x+\frac{M+1}{c_{1}}<\infty .
$$


Repeating the same arguments of the proof of (3.25) we obtain

$$
2 \int_{I_{1}} \phi\left(\mathbf{e}_{2}\right) \mathrm{d} \mathcal{H}^{1} \leqq \liminf _{k \rightarrow \infty} \int_{J_{v_{k}}} \phi\left(v_{J_{v_{k}}}\right) \mathrm{d} \mathcal{H}^{1} .
$$

Note that the direct application of Proposition 4.6 would not be enough since we would obtain the estimate:

$$
\int_{I_{1}} \phi\left(\mathbf{e}_{2}\right) \mathrm{d} \mathcal{H}^{1} \leqq \liminf _{k \rightarrow \infty} \int_{J_{v_{k}}} \phi\left(v_{J_{v_{k}}}\right) \mathrm{d} \mathcal{H}^{1}
$$

without coefficient 2 on the left.

From (4.34) and (4.35) it follows that there exists $k_{\delta}^{1}>0$ such that

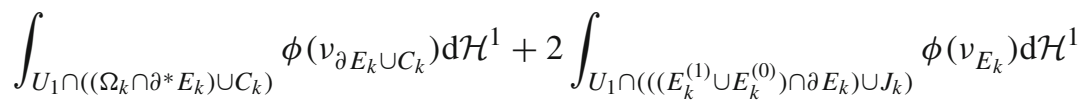

$$
\begin{aligned}
& \geqq 2 \int_{I_{1}} \phi\left(\mathbf{e}_{2}\right) \mathrm{d} \mathcal{H}^{1}-\frac{2 \delta}{5}
\end{aligned}
$$

for any $k \geqq k_{\delta}^{1}$. By (4.28) we may suppose that for such $k$,

$$
\int_{I_{1}} \phi\left(\mathbf{e}_{2}\right) \mathrm{d} \mathcal{H}^{1} \geqq \int_{U_{1} \cap \Sigma_{k}} \phi\left(v_{\Sigma_{k}}\right) \mathrm{d} \mathcal{H}^{1}-\frac{\delta}{5} .
$$

Thus, in view of (4.31), from (4.36) we get (4.30).

Step 2. Finally we prove (4.26). Let $k_{\delta / 2}^{1}$ be given by Step 1 with $\delta / 2$ in place of $\delta$. From (4.29) for $k>k_{\delta / 2}^{1}$ we have

$$
\begin{aligned}
& \int_{U_{1} \cap \Omega_{k} \cap \partial^{*} E_{k}} \phi\left(v_{E_{k}}\right) \mathrm{d} \mathcal{H}^{1}+2 \int_{U_{1} \cap \Omega_{k} \cap\left(E_{k}^{(1)} \cup E_{k}^{(0)}\right) \cap \partial E_{k}} \phi\left(v_{E_{k}}\right) \mathrm{d} \mathcal{H}^{1} \\
& \quad+\int_{U_{1} \cap \Sigma_{k} \backslash \partial E_{k}} g_{0} \mathrm{~d} \mathcal{H}^{1}+\int_{U_{1} \cap \Sigma_{k} \cap E_{k}^{(0)} \cap \partial E_{k}}\left(\phi\left(v_{\Sigma_{k}}\right)+g_{1}\right) \mathrm{d} \mathcal{H}^{1} \\
& \quad+\int_{U_{1} \cap \Sigma_{k} \cap \partial^{*} E_{k} \backslash J_{E_{k}}} g_{1} \mathrm{~d} \mathcal{H}^{1}+\int_{U_{1} \cap J_{E_{k}}}\left(\phi\left(v_{\Sigma_{k}}\right)+g_{0}\right) \mathrm{d} \mathcal{H}^{1} \\
& \geqq \int_{U_{1} \cap \Sigma_{k} \backslash \partial E_{k}}\left(\phi\left(v_{\Sigma_{k}}\right)+g_{0}\right) \mathrm{d} \mathcal{H}^{1}+\int_{U_{1} \cap \Sigma_{k} \cap E_{k}^{(0)} \cap \partial E_{k}}\left(2 \phi\left(v_{\Sigma_{k}}\right)+g_{1}\right) \mathrm{d} \mathcal{H}^{1} \\
& \quad+\int_{U_{1} \cap \Sigma_{k} \cap \partial^{*} E_{k} \backslash J_{E_{k}}}\left(2 \phi\left(v_{\Sigma_{k}}\right)+g_{1}\right) \mathrm{d} \mathcal{H}^{1}+\int_{U_{1} \cap J_{E_{k}}}\left(\phi\left(v_{\Sigma_{k}}\right)+g_{0}\right) \mathrm{d} \mathcal{H}^{1}-\frac{\delta}{2} .
\end{aligned}
$$

Thus, (4.28) implies that there exists $k_{\delta}>k_{\delta / 2}^{1}$ such that

$$
\begin{gathered}
\int_{U_{1} \cap \Sigma_{k} \backslash \partial E_{k}}\left(\phi\left(v_{\Sigma_{k}}\right)+g_{0}\right) \mathrm{d} \mathcal{H}^{1}+\int_{U_{1} \cap \Sigma_{k} \cap E_{k}^{(0)} \cap \partial E_{k}}\left(2 \phi\left(v_{\Sigma_{k}}\right)+g_{1}\right) \mathrm{d} \mathcal{H}^{1} \\
+\int_{U_{1} \cap \Sigma_{k} \cap \partial^{*} E_{k} \backslash J_{E_{k}}}\left(2 \phi\left(v_{\Sigma_{k}}\right)+g_{1}\right) \mathrm{d} \mathcal{H}^{1}+\int_{U_{1} \cap J_{E_{k}}}\left(\phi\left(v_{\Sigma_{k}}\right)+g_{0}\right) \mathrm{d} \mathcal{H}^{1}
\end{gathered}
$$




$$
\begin{aligned}
\geqq & \int_{I_{1} \cap \pi\left(\Sigma_{k} \backslash \partial E_{k}\right)}\left(\phi\left(\mathbf{e}_{2}\right)+g_{0}\right) \mathrm{d} \mathcal{H}^{1}+\int_{I_{1} \cap \pi\left(\Sigma_{k} \cap E_{k}^{(0)} \cap \partial E_{k}\right)}\left(2 \phi\left(\mathbf{e}_{2}\right)+g_{1}\right) \mathrm{d} \mathcal{H}^{1} \\
& +\int_{I_{1} \cap \pi\left(\Sigma_{k} \cap \partial^{*} E_{k} \backslash J_{E_{k}}\right)}\left(2 \phi\left(\mathbf{e}_{2}\right)+g_{1}\right) \mathrm{d} \mathcal{H}^{1}+\int_{I_{1} \cap \pi\left(J_{E_{k}}\right)}\left(\phi\left(\mathbf{e}_{2}\right)+g_{0}\right) \mathrm{d} \mathcal{H}^{1}-\frac{\delta}{2} \\
\geqq & \int_{I_{1}}\left(\phi\left(\mathbf{e}_{2}\right)+g_{0}\right) \mathrm{d} \mathcal{H}^{1}-\frac{\delta}{2},
\end{aligned}
$$

where we used also that $g_{0}$ and $g_{1}$ are constants. Now (4.26) follows from (4.37) and the last inequality.

Proof of Proposition 4.1. Without loss of generality, we suppose that the limit in the left-hand side of (4.3) is reached and finite. Define

$$
g_{+}(x, s)=g(x, s)-g(x, 0)+\varphi\left(x, v_{\Sigma}(x)\right) .
$$

Then $g_{+}$is Borel, $g_{+}(\cdot, s) \in L^{1}(\Sigma)$ for $s=0,1$, and by (4.1), $g_{+} \geqq 0$ and

$$
\left|g_{+}(x, 1)-g_{+}(x, 0)\right| \leqq \varphi\left(x, v_{\Omega}(x)\right)
$$

for $\mathcal{H}^{1}$-a.e. $x \in \Sigma$. Consider the sequence $\mu_{k}$ of Radon measures in $\mathbb{R}^{2}$, associated to $\mathcal{S}\left(A_{k}, J_{A_{k}} ; \varphi, g\right)$, defined at Borel sets $B \subset \mathbb{R}^{2}$ by

$$
\begin{aligned}
\mu_{k}(B):= & \int_{B \cap \Omega \cap \partial^{*} A_{k}} \varphi\left(x, v_{A_{k}}\right) \mathrm{d} \mathcal{H}^{1}+2 \int_{B \cap \Omega \cap\left(A_{k}^{(1)} \cup A_{k}^{(0)}\right) \cap \partial A_{k}} \varphi\left(x, v_{A_{k}}\right) \mathrm{d} \mathcal{H}^{1} \\
& +\int_{B \cap \Sigma \backslash \partial A_{k}} g_{+}(x, 0) \mathrm{d} \mathcal{H}^{1}+\int_{B \cap \Sigma \cap A_{k}^{(0)} \cap \partial A_{k}}\left(\varphi\left(x, v_{\Sigma}\right)+g_{+}(x, 1)\right) \mathrm{d} \mathcal{H}^{1} \\
& +\int_{B \cap \Sigma \cap \partial^{*} A_{k} \backslash J_{A_{k}}} g_{+}(x, 1) \mathrm{d} \mathcal{H}^{1}+\int_{B \cap J_{A_{k}}}\left(\varphi\left(x, v_{\Sigma}\right)+g_{+}(x, 0)\right) \mathrm{d} \mathcal{H}^{1} .
\end{aligned}
$$

Analogously, we define the positive Radon measure $\mu$ in $\mathbb{R}^{2}$ associated to $\mathcal{S}\left(A, J_{A} ; \varphi, g\right)$, writing $A$ in place of $A_{k}$ in the definition of $\mu_{k}$. By (2.4), assumption $A_{k} \stackrel{\tau_{\mathcal{A}}}{\rightarrow} A$ and the nonnegativity of $g_{+}$,

$$
\sup _{k \geqq 1} \mu_{k}\left(\mathbb{R}^{2}\right) \leqq 2 c_{2} \sup _{k \geqq 1} \mathcal{H}^{1}\left(\partial A_{k}\right)+\sum_{s=0}^{1} \int_{\Sigma} g_{+}(x, s) \mathrm{d} \mathcal{H}^{1}<\infty .
$$

Thus, by compactness there exists a (not relabelled) subsequence $\left\{\mu_{k}\right\}$ and a nonnegative bounded Radon measure $\mu_{0}$ in $\mathbb{R}^{2}$ such that $\mu_{k} \stackrel{*}{\rightarrow} \mu_{0}$ as $k \rightarrow \infty$. We claim that

$$
\mu_{0} \geqq \mu,
$$

which implies the assertion of the proposition. In fact, (4.3) follows from (4.39), the weak*-convergence of $\mu_{k}$, and the equalities

$$
\mu_{k}\left(\mathbb{R}^{2}\right)=\mathcal{S}\left(A_{k}, J_{A_{k}} ; \varphi, g\right)+\int_{\Sigma}\left(\varphi\left(x, v_{\Sigma}\right)-g(x, 0)\right) \mathrm{d} \mathcal{H}^{1}
$$


and

$$
\mu\left(\mathbb{R}^{2}\right)=\mathcal{S}\left(A, J_{A} ; \varphi, g\right)+\int_{\Sigma}\left(\varphi\left(x, v_{\Sigma}\right)-g(x, 0)\right) \mathrm{d} \mathcal{H}^{1}
$$

Since $\mu_{0}$ and $\mu$ are non-negative, and $\mu<<\mathcal{H}^{1} L(\partial A \cup \Sigma)$, by Remark 2.3 to prove (4.39) it suffices to establish the following lower-bound estimates for densities of $\mu_{0}$ with respect to $\mathcal{H}^{1}$ restricted to various parts of $\partial A$ :

$$
\begin{aligned}
& \frac{d \mu_{0}}{d \mathcal{H}^{1} \mathrm{~L}\left(\Omega \cap \partial^{*} A\right)}(x) \geqq \varphi\left(x, v_{A}(x)\right) \quad \text { for } \mathcal{H}^{1}-\text { almost everywhere } x \in \Omega \cap \partial^{*} A, \\
& \frac{d \mu_{0}}{d \mathcal{H}^{1} \mathrm{~L}\left(A^{(0)} \cap \partial A\right)}(x) \geqq 2 \varphi\left(x, v_{A}(x)\right) \quad \text { for } \mathcal{H}^{1}-\text { almost everywhere } x \in \Omega \cap A^{(0)} \cap \partial A, \\
& \frac{d \mu_{0}}{d \mathcal{H}^{1} \mathrm{~L}\left(A^{(1)} \cap \partial A\right)}(x) \geqq 2 \varphi\left(x, v_{A}(x)\right) \quad \text { for } \mathcal{H}^{1}-\text { almost everywhere } x \in \Omega \cap A^{(1)} \cap \partial A, \\
& \frac{d \mu_{0}}{d \mathcal{H}^{1} \mathrm{~L}(\Sigma \backslash \partial A)}(x)=g_{+}(x, 0) \quad \text { for } \mathcal{H}^{1}-\text { almost everywhere } x \in \Sigma \backslash \partial A \\
& \frac{d \mu_{0}}{d \mathcal{H}^{1} \mathrm{~L}\left(\Sigma \cap A^{(0)} \cap \partial A\right)}(x) \geqq \varphi\left(x, v_{\Sigma}(x)\right)+g_{+}(x, 1) \\
& \text { for } \mathcal{H}^{1}-\text { almost everywhere } x \in \Sigma \cap A^{(0)} \cap \partial A, \\
& \frac{\mathrm{d} \mu_{0}}{\mathrm{~d} \mathcal{H}^{1} \mathrm{~L}\left(\Sigma \cap \partial^{*} A\right)} \geqq g_{+}(x, 1) \quad \text { for } \mathcal{H}^{1}-\text { almost everywhere } x \in \Sigma \cap \partial^{*} A, \\
& \frac{d \mu_{0}}{d \mathcal{H}^{1} \mathrm{~L} J_{A}}(x) \geqq \varphi\left(x, v_{\Omega}(x)\right)+g_{+}(x, 0) \quad \text { for } \mathcal{H}^{1}-\text { almost everywhere } x \in J_{A} .
\end{aligned}
$$

We separately outline below the proofs of (4.40a)-(4.40g).

Proof of (4.40a). Consider points $x \in \Omega \cap \partial^{*} A$ such that

(a1) $v_{A}(x)$ exists;

(a2) $x$ is a Lebesgue point of $y \in \partial^{*} A \mapsto \varphi\left(y, v_{A}(y)\right)$, i.e.,

$$
\lim _{r \rightarrow 0} \frac{1}{2 r} \int_{U_{r} \cap \partial^{*} A}\left|\varphi\left(y, v_{A}(y)\right)-\varphi\left(x, v_{A}(x)\right)\right| \mathrm{d} \mathcal{H}^{1}(y)=0 ;
$$

(a3) $\frac{\mathrm{d} \mu_{0}}{\mathrm{~d} \mathcal{H}^{1} \mathrm{~L}\left(\Omega \cap \partial^{*} A\right)}(x)$ exists and is finite.

By the definition of $\partial^{*} A$, continuity of $\phi$, the Borel regularity of $y \in \partial^{*} A \mapsto$ $\varphi\left(y, v_{A}(y)\right)$, and the Besicovitch Derivation Theorem, the set of points $x \in \Omega \cap \partial^{*} A$ not satisfying these conditions is $\mathcal{H}^{1}$-negligible, hence we prove (4.40a) for $x \in$ $\Omega \cap \partial^{*} A$ satisfying (a1)-(a3). Without loss of generality we suppose $x=0$ and $v_{A}(x)=\mathbf{e}_{2}$. By Lemma $3.2, A_{k} \rightarrow A$ in $L^{1}\left(\mathbb{R}^{2}\right)$, therefore, $D \chi_{A_{k}} \stackrel{*}{\rightarrow} D \chi_{A}$, and hence, by the Besicovitch Derivation Theorem [3, Theorem 2.22] and the definition (2.1) of the reduced boundary,

$$
v_{A_{k}} \mathcal{H}^{1} \mathrm{~L}\left(\Omega \cap \partial^{*} A_{k}\right) \stackrel{*}{\rightarrow} v_{A} \mathcal{H}^{1} \mathrm{~L}\left(\Omega \cap \partial^{*} A\right) .
$$

Then for a.e. $r>0$ such that $U_{r} \subset \subset \Omega$ and $\mathcal{H}^{1}\left(\partial U_{r} \cap \partial A\right)=0$, the Reshetnyak Lower-semicontinuity Theorem [3, Theorem 2.38] implies

$\mu_{0}\left(U_{r}\right)=\liminf _{k \rightarrow \infty} \mu_{k}\left(U_{r}\right) \geqq \liminf _{k \rightarrow \infty} \int_{U_{r} \cap \partial^{*} A_{k}} \varphi\left(y, v_{A_{k}}\right) \mathrm{d} \mathcal{H}^{1} \geqq \int_{U_{r} \cap \partial^{*} A} \varphi\left(y, v_{A}\right) \mathrm{d} \mathcal{H}^{1}$ 
Therefore, by [32, Theorem 1.153] and assumption (a2),

$\frac{\mathrm{d} \mu_{0}}{\mathrm{~d} \mathcal{H}^{1} \mathrm{~L}\left(\Omega \cap \partial^{*} A\right)}(0)=\lim _{r \rightarrow 0} \frac{\mu_{0}\left(U_{r}\right)}{2 r} \geqq \liminf _{r \rightarrow 0} \frac{1}{2 r} \int_{U_{r} \cap \partial^{*} A} \varphi\left(y, v_{A}\right) \mathrm{d} \mathcal{H}^{1}=\varphi\left(0, \mathbf{e}_{2}\right)$.

Proof of (4.40b). Consider points $x \in \Omega \cap A^{(0)} \cap \partial A$ such that

(b1) $\theta^{*}(\partial A, x)=\theta_{*}(\partial A, x)=1$;

(b2) $v_{A}(x)$ exists;

(b3) $\overline{U_{1}} \cap \sigma_{\rho, x}(\partial A) \stackrel{\mathcal{K}}{\rightarrow} \overline{U_{1}} \cap T_{x}$, where $T_{x}$ is the approximate tangent line to $\partial A$ and $\sigma_{\rho, x}$ is given by (4.4);

(b4) $\frac{\mathrm{d} \mu_{0}}{\mathrm{~d} \mathcal{H}^{1}\left\llcorner\left(A^{(0)} \cap \partial A\right)\right.}$ exists and finite.

By the $\mathcal{H}^{1}$-rectifiability of $\partial A$, Proposition A.4 (applied with the closed connected component $K$ of $\partial A$ containing $x$ ) and the Besicovitch Derivation Theorem, the set of points $x \in A^{(0)} \cap \partial A$ not satisfying these conditions is $\mathcal{H}^{1}$-negligible, hence we prove (4.40b) for $x \in A^{(0)} \cap \partial A$ satisfying (b1)-(b4). Without loss of generality we assume $x=0, v_{A}(x)=\mathbf{e}_{2}$ and $T_{x}=T_{0}$ is the $x_{1}$-axis.

Let us choose a sequence $\rho_{n} \searrow 0$ such that

$$
\mu_{0}\left(\partial U_{\rho_{n}}\right)=0 \quad \text { and } \quad \lim _{k \rightarrow \infty} \mu_{k}\left(\overline{U_{\rho_{n}}}\right)=\mu_{0}\left(U_{\rho_{n}}\right)
$$

and

$$
\frac{\mathrm{d} \mu_{0}}{\mathrm{~d} \mathcal{H}^{1} \mathrm{~L}\left(A^{(0)} \cap \partial A\right)}(0)=\lim _{n \rightarrow \infty} \frac{\mu_{0}\left(U_{\rho_{n}}\right)}{2 \rho_{n}} .
$$

By Proposition A.5 (a), (b2) and (b3) imply that $\operatorname{sdist}\left(\cdot, \sigma_{\rho_{n}}(\partial A)\right) \rightarrow \operatorname{dist}\left(\cdot, T_{0}\right)$ uniformly in $\overline{U_{1}}$. Since for any $n>1$, $\operatorname{sdist}\left(\cdot, \partial A_{k}\right) \rightarrow \operatorname{sdist}(\cdot, \partial A)$ uniformly in $\overline{U_{\rho_{n}}}$ as $k \rightarrow \infty$, by a diagonal argument, we find a subsequence $\left\{A_{k_{n}}\right\}$ such that

$$
\operatorname{sdist}\left(\cdot, \sigma_{\rho_{n}}\left(\partial A_{k_{n}}\right)\right) \rightarrow \operatorname{dist}\left(\cdot, T_{0}\right) \quad \text { uniformly in } \overline{U_{1}},
$$

as $n \rightarrow \infty$ and

$$
\mu_{k_{n}}\left(\overline{U_{\rho_{n}}}\right) \leqq \mu_{0}\left(U_{\rho_{n}}\right)+\rho_{n}^{2}
$$

for any $n$. By Lemma $4.2, \overline{U_{1}} \cap \sigma_{\rho_{n}}\left(A_{k_{n}}\right) \stackrel{\mathcal{K}}{\rightarrow} I_{1}:=\overline{U_{1}} \cap T_{0}$.

From (2.4), (4.43), the definition of $\mu_{k},(4.42)$ and (b4) it follows that

$$
\limsup _{n \rightarrow \infty} \frac{\mathcal{H}^{1}\left(\overline{U_{\rho}} \cap \partial A_{k_{n}}\right)}{2 \rho_{n}} \leqq \limsup _{n \rightarrow \infty} \frac{\mu_{k_{n}}\left(\overline{U_{\rho_{n}}}\right)}{2 c_{1} \rho_{n}} \leqq c_{1}^{-1} \frac{\mathrm{d} \mu_{0}}{\mathcal{H}^{1} \mathrm{~L}\left(A^{(0)} \cap \partial A\right)}(0) .
$$

By the uniform continuity of $\varphi$, for every $\epsilon>0$ there exists $n_{\epsilon}>0$ such that

$$
\varphi(y, \xi) \geqq \varphi(0, \xi)-\epsilon
$$

for every $y \in U_{\rho_{n_{\epsilon}}}$. Moreover, since $\left\{A_{k}\right\} \subset \mathcal{A}_{m}$, the number of connected components of $\partial \sigma_{\rho_{n}}\left(A_{k_{n}}\right)$ lying strictly inside $U_{1}$, does not exceed from $m$. Hence, 
applying Lemma 4.4 with $\phi=\varphi(0, \cdot), m_{o}=m$ and $\delta=\epsilon$, we find $n_{\epsilon}^{\prime}>n_{\epsilon}$ such that for any $n>n_{\epsilon}^{\prime}$,

$$
\begin{aligned}
& \int_{U_{1} \cap \partial^{*} \sigma_{\rho_{n}}\left(A_{k_{n}}\right)} \varphi\left(0, v_{\sigma_{\rho_{n}}\left(A_{k_{n}}\right)}\right) \mathrm{d} \mathcal{H}^{1} \\
& \quad+2 \int_{U_{1} \cap\left(\left(\sigma_{\rho_{n}}\left(A_{k_{n}}\right)\right)^{(0)} \cup\left(\sigma_{\rho_{n}}\left(A_{k_{n}}\right)\right)^{(1)}\right) \cap \partial \sigma_{\rho_{n}}\left(A_{k_{n}}\right)} \varphi\left(0, v_{\sigma_{\rho_{n}}\left(A_{k_{n}}\right)}\right) \mathrm{d} \mathcal{H}^{1} \\
& \quad \geqq 2 \int_{I_{1}} \varphi\left(0, \mathbf{e}_{2}\right) \mathrm{d} \mathcal{H}^{1}-\epsilon=4 \varphi\left(0, \mathbf{e}_{2}\right)-\epsilon .
\end{aligned}
$$

Therefore, by the definition of $\mu_{k}$, for such $n$ one has

$$
\begin{aligned}
\mu_{k_{n}}\left(\overline{U_{\rho_{n}}}\right) \geqq & \int_{\overline{U_{\rho_{n}}} \cap \partial^{*} A_{k_{n}}} \varphi\left(y, v_{A_{k_{n}}}\right) \mathrm{d} \mathcal{H}^{1}+2 \int_{\overline{U_{\rho_{n}}} \cap\left(A_{k}^{(0)} \cup A_{k}^{(1)}\right) \cap \partial A_{k_{n}}} \varphi\left(y, v_{A_{k_{n}}}\right) \mathrm{d} \mathcal{H}^{1} \\
\geqq & \int_{\overline{U_{\rho_{n}}} \cap \partial^{*} A_{k_{n}}} \varphi\left(0, v_{A_{k_{n}}}\right) \mathrm{d} \mathcal{H}^{1}+2 \int_{\overline{U_{\rho_{n}}} \cap\left(A_{k}^{(0)} \cup A_{k}^{(1)}\right) \cap \partial A_{k_{n}}} \varphi\left(0, v_{A_{k_{n}}}\right) \mathrm{d} \mathcal{H}^{1} \\
& -\epsilon \mathcal{H}^{1}\left(\overline{U_{\rho_{n}}} \cap \partial A_{k}\right) \\
= & \rho_{n}\left(\int_{U_{1} \cap \partial^{*} \sigma_{\rho_{n}}\left(A_{k_{n}}\right)} \varphi\left(0, v_{\sigma_{\rho_{n}}\left(A_{k_{n}}\right)}\right) \mathrm{d} \mathcal{H}^{1}\right. \\
& \left.+2 \int_{U_{1} \cap\left(\left(\sigma_{\rho_{n}}\left(A_{k_{n}}\right)\right)^{(0)} \cup\left(\sigma_{\rho_{n}}\left(A_{k_{n}}\right)\right)^{(1)}\right) \cap \partial \sigma_{\rho_{n}}\left(A_{k_{n}}\right)} \varphi\left(0, v_{\sigma_{\rho_{n}}\left(A_{k_{n}}\right)}\right) \mathrm{d} \mathcal{H}^{1}\right) \\
& -\epsilon \mathcal{H}^{1}\left(\overline{U_{\rho_{n}}} \cap \partial A_{k_{n}}\right) \\
\geqq & 4 \rho_{n} \varphi\left(0, \mathbf{e}_{2}\right)-\epsilon \rho_{n}-\epsilon \mathcal{H}^{1}\left(\overline{U_{\rho_{n}}} \cap \partial A_{k_{n}}\right),
\end{aligned}
$$

and thus, by (4.42)-(4.44),

$$
\begin{aligned}
\frac{\mathrm{d} \mu_{0}}{\mathrm{~d} \mathcal{H}^{1}\left\llcorner\left(A^{(0)} \cap \partial A\right)\right.}(0) & \geqq \liminf _{n \rightarrow \infty} \frac{\mu_{k_{n}}\left(\overline{U_{\rho_{n}}}\right)}{2 \rho_{n}} \\
& \geqq 2 \varphi\left(0, \mathbf{e}_{2}\right)-\frac{\epsilon}{2}-\epsilon \limsup _{n \rightarrow \infty} \frac{\mathcal{H}^{1}\left(\overline{U_{\rho_{n}}} \cap \partial A_{k}\right)}{2 \rho_{n}} \\
& \geqq 2 \varphi\left(0, \mathbf{e}_{2}\right)-\frac{\epsilon}{2}-c_{1}^{-1} \epsilon \frac{\mathrm{d} \mu_{0}}{\mathrm{~d} \mathcal{H}^{1} L\left(A^{(0)} \cap \partial A\right)}(0)
\end{aligned}
$$

Now using assumption (b4) and letting $\epsilon \rightarrow 0^{+}$we obtain (4.40b).

Proof of (4.40c). We repeat the same arguments of the proof of (4.40b) using Lemma 4.5 in place of Lemma 4.4 and Proposition A.5 (a) in place of Proposition A.5 (b).

Proof of (4.40d). Given $x \in \Sigma \backslash \partial A$, there exists $r_{x}>0$ such that $B_{r_{x}}(x) \cap \partial A=$ $\emptyset$. Since $\partial A_{k} \stackrel{\mathcal{K}}{\rightarrow} \partial A$, there exists $k_{x}$ such that $B_{r_{x} / 2}(x) \cap \partial A_{k}=\emptyset$ for all $k>k_{x}$. Thus, for any $r \in\left(0, r_{x} / 2\right)$,

$$
\mu_{k}\left(B_{r}(x)\right)=\int_{\Sigma \cap B_{r}(x)} g_{+}(y, 0) \mathrm{d} \mathcal{H}^{1}
$$


so that

$$
\frac{d \mu_{0}}{d \mathcal{H}^{1}\llcorner(\Sigma \backslash \partial A)}(x)=g_{+}(x, 0)
$$

for $\mathcal{H}^{1}$-a.e. Lebesgue points $x \in \Sigma \backslash \partial A$ of $g_{+}$.

Proof of (4.40e). Consider points $x \in \Sigma \cap A^{(0)} \cap \partial A$ such that

(e1) $\theta^{*}(\Sigma \cap \partial A, x)=\theta_{*}(\Sigma \cap \partial A, x)=1$;

(e2) $v_{\Sigma}(x)$ and $v_{A}(x)$ exist (clearly, either $v_{\Sigma}(x)=v_{A}(x)$ or $v_{\Sigma}(x)=-v_{A}(x)$ );

(e3) $\overline{U_{1}} \cap \sigma_{\rho, x}(\partial A) \stackrel{\mathcal{K}}{\rightarrow} \overline{U_{1}} \cap T_{x}$, where $T_{x}$ is the approximate tangent line to $\partial A$;

(e4) $x$ is a Lebesgue point of $g_{+}(\cdot, 1)$, i.e.,

$$
\lim _{\rho \rightarrow 0} \frac{1}{2 \rho} \int_{\Sigma \cap U_{\rho, v_{\Sigma}(x)}}\left|g_{+}(y, 1)-g_{+}(x, 1)\right| \mathrm{d} \mathcal{H}^{1}(y)=0 ;
$$

(e5) $x$ is a Lebesgue point of $y \in \Sigma \cap \varphi\left(y, v_{\Sigma}(y)\right)$, i.e.,

$$
\lim _{\rho \rightarrow 0} \frac{1}{2 \rho} \int_{\Sigma \cap U_{\rho, v_{\Sigma}(x)}}\left|\varphi\left(y, v_{\Sigma}(y)\right)-\varphi\left(x, v_{\Sigma}(x)\right)\right| \mathrm{d} \mathcal{H}^{1}(y)=0 ;
$$

(e6) $\frac{d \mu_{0}}{d \mathcal{H}^{1} \mathrm{~L} \Sigma}(x)$ exists and is finite.

By the $\mathcal{H}^{1}$-rectifiability of $\partial A$, the Lipschitz continuity of $\Sigma$, the Borel regularity of $v_{\Sigma}(\cdot)$, Proposition A.4 (applied with closed connected component $K$ of $\partial A$ containing $x$ ), the continuity of $\varphi$, assumptions on $g_{+}$and the Besicovitch Derivation Theorem, the set of $x \in \Sigma \cap \partial A$ not satisfying these conditions is $\mathcal{H}^{1}$-negligible. Hence, we prove (4.40e) for $x$ satisfying (e1)-(e6). Without loss of generality we assume $x=0, v_{\Sigma}(x)=v_{A}(x)=\mathbf{e}_{2}$ and $T_{x}=T_{0}$ is the $x_{1}$-axis. Let $r_{n} \searrow 0$ be such that

$$
\mu_{0}\left(\partial U_{r_{n}}\right)=\mathcal{H}^{1}\left(\partial U_{r_{n}} \cap \Sigma\right)=0
$$

and

$$
\frac{d \mu_{0}}{d \mathcal{H}^{1}\left\llcorner\left(\Sigma \cap A^{(0)} \cap \partial A\right)\right.}(x)=\lim _{n \rightarrow \infty} \frac{\mu_{0}\left(U_{r_{n}}\right)}{2 r_{n}} .
$$

By the weak*-convergence, for any $h \geqq 1$ we have

$$
\lim _{k \rightarrow \infty} \mu_{k}\left(\overline{U_{r_{n}}}\right)=\mu_{0}\left(U_{r_{n}}\right)
$$

By Proposition A.5 (b), (e2) and (e3) imply $\operatorname{sdist}\left(\cdot, \sigma_{r_{n}}(\partial A)\right) \rightarrow \operatorname{dist}\left(\cdot, T_{0}\right)$ uniformly in $\overline{U_{1}}$. Since for any $n, \operatorname{sdist}\left(\cdot, \sigma_{r_{n}}\left(\partial A_{k}\right)\right) \rightarrow \operatorname{sdist}\left(\cdot, \sigma_{r_{n}}(\partial A)\right)$ uniformly in $\overline{U_{1}}$ as $k \rightarrow \infty$, by a diagonal argument, we can find a subsequence $\left\{k_{n}\right\}$ and not relabelled subsequence $\left\{r_{n}\right\}$ such that

$$
\mu_{k_{n}}\left(\overline{U_{r_{n}}}\right) \leqq \mu_{0}\left(U_{r_{n}}\right)+r_{n}^{2}
$$


for any $n \geqq 1$ and $\operatorname{sdist}\left(\cdot, \sigma_{r_{n}}\left(A_{k}\right)\right) \rightarrow \sigma\left(\cdot, T_{0}\right)$ uniformly in $\overline{U_{1}}$ as $k \rightarrow \infty$, thus, by Lemma 4.2 ,

$$
U_{1} \cap \sigma_{r_{n}}\left(A_{k_{n}}\right) \stackrel{\mathcal{K}}{\rightarrow} I_{1}:=U_{1} \cap T_{0}
$$

as $n \rightarrow \infty$. Notice also that by (e2) and Proposition A.4 (applied with the closed connected component $K$ of $\Sigma), U_{1} \cap \sigma_{r_{n}}(\Sigma) \stackrel{\mathcal{K}}{\rightarrow} I_{1}$ as $n \rightarrow \infty$.

By (4.38),

$$
\varphi\left(y, v_{\Sigma}(x)\right)+g_{+}(y, 0) \geqq g_{+}(y, 1)
$$

for $\mathcal{H}^{1}$-a.e. on $\Sigma$, in particular on $J_{A_{k}}$, hence, by Remark 2.3 and the definition of $\mu_{k}$,

$$
\begin{aligned}
\mu_{k_{n}}\left(\overline{U_{r_{n}}}\right) \geqq & \int_{\overline{U_{r_{n}}} \cap \Omega \cap \partial^{*} A_{k_{n}}} \varphi\left(y, v_{A_{k_{n}}}\right) \mathrm{d} \mathcal{H}^{1}+2 \int_{\overline{U_{r_{n}}} \cap \Omega \cap\left(A_{k_{n}}^{(0)} \cup A_{k_{n}}^{(1)}\right) \cap \partial A_{k_{n}}} \varphi\left(y, v_{A_{k_{n}}}\right) \mathrm{d} \mathcal{H}^{1} \\
& +\int_{U_{r_{n}} \cap \Sigma} g_{+}(y, 1) \mathrm{d} \mathcal{H}^{1}+\int_{U_{r_{n}} \cap \Sigma \cap A_{k_{n}}^{(0)} \cap \partial A_{k_{n}}} \varphi\left(y, v_{\Sigma}\right) \mathrm{d} \mathcal{H}^{1} \\
& +\int_{U_{r_{n}} \cap \Sigma \backslash \partial A_{k_{n}}}\left(g_{+}(y, 0)-g_{+}(y, 1)\right) \mathrm{d} \mathcal{H}^{1}
\end{aligned}
$$

Adding and subtracting $\int \overline{U_{r_{n}}} \cap \Sigma \cap \partial^{*} A_{k_{n}} \phi\left(y, v_{A_{k_{n}}}\right) \mathrm{d} \mathcal{H}^{1}$ to the right and using (4.38) once more in the integral over $U_{r_{n}} \cap \Sigma \backslash \partial A_{k_{n}}$ we get

$$
\begin{aligned}
\mu_{k_{n}}\left(\overline{U_{r_{n}}}\right) \geqq & \int_{\overline{U_{r_{n}}} \cap \partial^{*} A_{k_{n}}} \varphi\left(y, v_{A_{k_{n}}}\right) \mathrm{d} \mathcal{H}^{1}+2 \int_{\overline{U_{r_{n}}} \cap\left(A_{k_{n}}^{(0)} \cup A_{k_{n}}^{(1)}\right) \cap \partial A_{k_{n}}} \varphi\left(y, v_{A_{k_{n}}}\right) \mathrm{d} \mathcal{H}^{1} \\
& +\int_{U_{r_{n}} \cap \Sigma} g_{+}(y, 1) \mathrm{d} \mathcal{H}^{1}-\int_{U_{r_{n}} \cap \Sigma} \varphi\left(y, v_{\Sigma}(y)\right) \mathrm{d} \mathcal{H}^{1}
\end{aligned}
$$

By the uniform continuity of $\varphi$, given $\epsilon \in(0,1)$ there exists $n_{\epsilon}>0$ such that

$$
|\varphi(y, v)-\varphi(0, v)|<\epsilon
$$

for all $y \in U_{r_{n}}, v \in \mathbb{S}^{1}$ and $n>n_{\epsilon}$. We suppose also that Lemma 4.4 holds with $n_{\epsilon}$ when $\delta=\epsilon$. Since the number of connected components of $\partial A_{k_{n}}$ lying strictly inside $U_{r_{n}}$ is not greater than $m$, in view of (4.49) and the non-negativity of $g_{+}$, as in (4.46) for all $n>n_{\epsilon}$ we obtain

$$
\begin{aligned}
\mu_{k_{n}}\left(\overline{U_{r_{n}}}\right) \geqq & 4 r_{n} \varphi\left(0, \mathbf{e}_{2}\right)-\epsilon r_{n}-\epsilon \mathcal{H}^{1}\left(\overline{U_{r_{n}} \cap \partial A_{k_{n}}}\right) \\
& +\int_{U_{r_{n}} \cap \Sigma} g_{+}(y, 1) \mathrm{d} \mathcal{H}^{1}-\int_{U_{r_{n}} \cap \Sigma} \varphi\left(y, v_{\Sigma}(y)\right) \mathrm{d} \mathcal{H}^{1} .
\end{aligned}
$$

By the non-negativity of $g_{+},(2.4)$ and (4.48),

$$
\begin{aligned}
\mathcal{H}^{1}\left(\overline{U_{r_{n}} \cap \partial A_{k_{n}}}\right) & \leqq \mathcal{H}^{1}\left(U_{r_{n}} \cap \Omega \cap \cap \partial A_{k_{n}}\right)+\mathcal{H}^{1}\left(U_{r_{n}} \cap \Sigma \cap \cap \partial A_{k_{n}}\right) \\
\leqq & \frac{\mu_{k_{n}}\left(U_{r_{n}}\right)}{c_{1}}+\mathcal{H}^{1}\left(U_{r_{n}} \cap \Sigma\right) \leqq \mu_{0}\left(U_{r_{n}}\right)+r_{n}^{2}+\mathcal{H}^{1}\left(U_{r_{n}} \cap \Sigma\right)
\end{aligned}
$$


thus again using (4.48), also (4.47), (4.50) and (4.51), as well as (e1) and (e3)-(e5) we establish

$$
\begin{aligned}
& \frac{\mathrm{d} \mu_{0}}{\mathrm{~d} \mathcal{H}^{1} \mathrm{~L}\left(\Sigma \cap A^{(0)} \cap \partial A\right)}(0)=\lim _{h \rightarrow \infty} \frac{\mu_{0}\left(U_{r_{n}}\right)}{2 r_{n}} \geqq \limsup _{h \rightarrow \infty} \frac{\mu_{k_{n}}\left(U_{r_{n}}\right)}{2 r_{n}} \\
& \geqq 2 \varphi\left(0, \mathbf{e}_{2}\right)-\frac{3 \epsilon}{2}-\epsilon \frac{\mathrm{d} \mu_{0}}{\mathrm{~d} \mathcal{H}^{1} \mathrm{~L}\left(\Sigma \cap A^{(0)} \cap \partial A\right)}(0)+g_{+}(0,1) \mathrm{d} \mathcal{H}^{1}-\varphi\left(0, v_{\Sigma}(0)\right) .
\end{aligned}
$$

Now letting $\epsilon \rightarrow 0$ and using $\nu_{\Sigma}(0)=\mathbf{e}_{2}$ we obtain (4.40e).

Proof of (4.40f). Since $g_{+}$is non-negative and $\chi_{A_{k}} \rightarrow \chi_{A}$ in $L^{1}\left(\mathbb{R}^{2}\right)$, the inequality follows from [1, Lemma 3.8] applied to $u_{k}=\chi_{A_{k}}, u=\chi_{A}$ and

$$
\mathcal{F}_{U}(v, B):=\int_{U \cap B \cap J_{v} \backslash \Sigma} \varphi\left(x, v_{J_{v}}\right) \mathrm{d} \mathcal{H}^{1}+\int_{\Sigma \cap B} g_{+}(x, 1) v^{+}(x) \mathrm{d} \mathcal{H}^{1},
$$

where $U:=\Omega$. More precisely, by (4.1),

$$
\mu_{k}(B) \geqq \mathcal{F}_{U}\left(\chi_{A_{k}}, B\right)
$$

so that the total variation of $\mathcal{F}_{U}\left(\chi_{A_{k}}, \cdot\right)$ is uniformly bounded. Therefore, passing to a further not relabelled subsequence if necessary, we have $\mathcal{F}_{U}\left(\chi_{A_{k}}, \cdot\right) \rightarrow^{*} \bar{\mu}$ as $k \rightarrow$ $\infty$ for some bounded positive Radon measure $\bar{\mu}$ in $\mathbb{R}^{2}$. By [1, Lemma 3.8, Eq. 3.15],

$$
\frac{d \bar{\mu}}{\mathrm{d} \mathcal{H}^{1}\llcorner\Sigma}(x) \geqq g_{+}(x, 1) \chi_{\partial^{*} A} \quad \text { for } \mathcal{H}^{1}-\text { almost everywhere } x \in \Sigma
$$

By (4.52), $\mu_{0} \geqq \bar{\mu}$, and thus, (4.40f) follows.

Proof of (4.40g). Consider points $x \in J_{A}$ for which

(g1) $\theta^{*}\left(J_{A}, x\right)=\theta_{*}\left(J_{A}, x\right)=\theta^{*}(\partial A, x)=\theta_{*}(\partial A, x)=\theta^{*}(\Sigma, x)=\theta_{*}(\Sigma, x)=$ 1 ;

(g2) assumption (b) of Proposition 4.1 holds with some $r=r_{x}>0$;

(g3) $\Sigma$ is differentiable at $x$ and $v_{\Sigma}(x)$ exists;

(g4) one-sided traces $w^{+}(x) \neq w^{-}(x)$ of $w$, given by assumption (b) of Proposition 4.1, exists;

(g5) $x$ is a Lebesgue point of $g_{+}(\cdot, s)$ and $\left|g_{+}(x, 0)-g_{+}(x, 1)\right| \leqq \phi\left(v_{\Sigma}(x)\right)$;

(g6) $\frac{\mathrm{d} \mu_{0}}{\mathrm{~d} \mathcal{H}^{1}\left[J_{A}\right.}(x)$ exists and finite.

By the $\mathcal{H}^{1}$-rectifiability of $J_{A}, \partial A$ and $\Sigma$, assumption (b) of Proposition 4.1 (recall that $J_{A} \subset J_{w}$ ), the definition of the jump set of $G S B D$-functions, (4.38), and the Besicovitch Derivation Theorem, the set of points $x \in J_{A}$ not satisfying these conditions is $\mathcal{H}^{1}$-negligible. Hence we prove (4.40g) for $x \in J_{A}$ satisfying (g1)(g6). Without loss of generality, we assume $x=0$ and $v_{\Sigma}(x)=\mathbf{e}_{2}$. Let $r_{0}=r_{x}$ and $w_{k} \in G S B D^{2}\left(B_{r_{0}}(0) ; \mathbb{R}^{2}\right)$ be given by assumption (b) of Proposition 4.1. Note that by the weak ${ }^{*}$-convergence of $\mu_{k}$,

$$
\lim _{k \rightarrow \infty} \mu_{k}\left(\overline{U_{r}}\right)=\mu_{0}\left(U_{r}\right)
$$


for a.e. $r \in\left(0, r_{0}\right)$, and by $(\mathrm{g} 1),(\mathrm{g} 3)$, and Proposition A.4 (applied with connected components of $\Sigma$ and $\partial A$ intersecting at $x$ ) and also by the definition of blow-up,

$$
U_{4} \cap \sigma_{r}(\Sigma) \stackrel{\mathcal{K}}{\rightarrow} I_{4} \quad \text { and } \quad \mathcal{H}^{1} \mathrm{~L}\left(U_{4} \cap \sigma_{r}(\Sigma)\right) \stackrel{*}{\rightarrow} \mathcal{H}^{1}\left\llcorner I_{4}\right.
$$

and

$$
U_{4} \cap \sigma_{r}(\partial A) \stackrel{\mathcal{K}}{\rightarrow} I_{4} \quad \text { and } \quad \mathcal{H}^{1} \mathrm{~L}\left(U_{4} \cap \sigma_{r_{h}}(\partial A)\right) \stackrel{*}{\rightarrow} \mathcal{H}^{1}\left\llcorner I_{4}\right.
$$

as $r \rightarrow 0$. Since $J_{A} \subset \Sigma$, in view of (4.54),

$$
U_{4} \cap \sigma_{r}\left(J_{A}\right) \stackrel{\mathcal{K}}{\rightarrow} I_{4} .
$$

Moreover, since $J_{A}$ has a generalized normal at $x=0$,

$$
\mathcal{H}^{1} \mathrm{~L}\left(U_{4} \cap \sigma_{r}\left(J_{A}\right)\right) \stackrel{*}{\rightarrow} \mathcal{H}^{1}\left\llcorner I_{4}\right.
$$

as $r \rightarrow 0$. In particular, from (4.54) and (4.55),

$$
\operatorname{sdist}\left(\cdot, U_{4} \cap \sigma_{r}(\partial \Omega)\right) \rightarrow \operatorname{sdist}\left(\cdot, \partial U_{4}^{+}\right)
$$

and

$$
\operatorname{sdist}\left(\cdot, U_{4} \cap \sigma_{r}(\partial A)\right) \rightarrow \operatorname{sdist}\left(\cdot, \partial U_{4}^{+}\right)
$$

locally uniformly in $U_{3 / 2}$ as $r \rightarrow 0$.

Letting $\phi=\varphi(0, \cdot)$, we claim that there exist sequences $r_{h} \searrow 0$ and $k_{h} \nearrow \infty$ such that the sets

$$
\Omega_{h}:=U_{4} \cap \sigma_{r_{h}}(\Omega), \quad \Sigma_{h}:=U_{4} \cap \sigma_{r_{h}}(\Sigma),
$$

and

$$
E_{h}:=U_{4} \cap \sigma_{r_{h}}\left(A_{k_{h}}\right), \quad J_{E_{h}}=U_{4} \cap \sigma_{r_{h}}\left(J_{A_{k_{h}}}\right),
$$

the functions $u_{h}(x)=w_{k_{h}}\left(r_{h} x\right) \in G S B D^{2}\left(U_{4} ; \mathbb{R}^{2}\right)$, the numbers $g_{s}=g_{+}(0, s) \in$ $[0,+\infty)$ and the vectors $u^{ \pm}=w^{ \pm}(0)$ satisfy assumptions (a)-(h) of Lemma 4.7.

Indeed, let $\tau$ be any homeomorphism between $\mathbb{R}^{2}$ and a bounded subset of $\mathbb{R}^{2}$; for example, one can take $\tau\left(x_{1}, x_{2}\right)=\left(\tanh \left(x_{1}\right), \tanh \left(x_{2}\right)\right)$. By $(4.2), w_{k}(r x) \rightarrow$ $w(r x)$ as $k \rightarrow \infty$ for a.e. $x \in U_{4}$ and for any $r \in\left(0, r_{0} / 4\right)$, so that by the Dominated Convergence Theorem,

$$
\lim _{k \rightarrow \infty} \int_{U_{1}}\left|\tau\left(w_{k}(r x)\right)-\tau(w(r x))\right| \mathrm{d} x=0 .
$$

Moreover, by (g4), the definition [19, Definition 2.4] of the (approximate) jump of the function $w$ and [19, Remark 2.2],

$$
\lim _{r \rightarrow 0} \int_{U_{1}} \mid \tau(w(r x))-(\tau(u(x)) \mid \mathrm{d} x=0,
$$


where $u(x):=w^{+}(0) \chi_{U_{1}^{+}}(x)+w^{-}(0) \chi_{U_{1} \backslash U_{1}^{+}}(x)$. We use (4.60) and (4.61) to extract sequences $k_{h} \rightarrow \infty$ and $r_{h} \rightarrow 0$ such that $w_{k_{h}}\left(r_{h} x\right) \rightarrow u(x)$ a.e. in $U_{1}$. By assumption $A_{k} \stackrel{\tau_{\mathcal{A}}}{\rightarrow} A$ and the relations (4.59), (4.53), (4.61) and (4.60), there exist $k_{h}^{1}>1$ and a decreasing sequence $r_{h} \in\left(0, \frac{1}{h}\right)$ such that for any $h>1$ and $k>k_{h}^{1}$,

$$
\begin{aligned}
& \left\|\operatorname{sdist}\left(\cdot, \sigma_{r_{h}}\left(U_{4 r_{h}} \cap \partial A_{k}\right)\right)-\operatorname{sdist}\left(\cdot, \sigma_{r_{h}}\left(U_{4 r_{h}} \cap \partial A\right)\right)\right\|_{L^{\infty}\left(U_{3 / 2}\right)}<\frac{1}{h} \\
& \left\|\operatorname{sdist}\left(\cdot, \sigma_{r_{h}}\left(U_{4 r_{h}} \cap \partial A\right)\right)-\operatorname{sdist}\left(\cdot, \partial U_{4}^{+}\right)\right\|_{L^{\infty}\left(U_{3 / 2}\right)}<\frac{1}{h} \\
& \mu_{k}\left(\overline{U_{r_{h}}}\right)<\mu_{0}\left(U_{r_{h}}\right)+r_{h}^{2} \\
& \int_{U_{1}}\left|\tau\left(w\left(r_{h} x\right)\right)-\tau(u(x))\right| \mathrm{d} x<\frac{1}{h} \\
& \int_{U_{1}}\left|\tau\left(w_{k}\left(r_{h} x\right)\right)-\tau\left(w\left(r_{h} x\right)\right)\right| \mathrm{d} x<\frac{1}{h} .
\end{aligned}
$$

For every $h \geqq 1$, we choose $k_{h}>k_{h}^{1}$ such that

$$
\frac{1}{k_{h} r_{h}}<\frac{1}{h} \text {. }
$$

Now $u_{h}(x):=w_{k_{h}}\left(r_{h} x\right) \in G S B D^{2}\left(U_{2} ; \mathbb{R}^{2}\right)$, and

- by (4.58), $\operatorname{sdist}\left(\cdot, U_{4} \cap \partial \Omega_{h}\right) \rightarrow \operatorname{sdist}\left(\cdot, \partial U_{4}^{+}\right)$locally uniformly in $U_{3 / 2}$ as $h \rightarrow \infty$;

- by assumption (g3) and the Lipschitz property of $\Sigma, U_{4 r_{h}} \cap \Sigma$ is a graph of a $L$-Lipschitz function $l:\left[-4 r_{h}, 4 r_{h}\right] \rightarrow \mathbb{R}$ so that $\Sigma_{h}=U_{4} \cap \partial \Omega_{h}$ is the graph of $l_{h}(t):=l\left(r_{h} t\right)$, where $t \in[-4,4]$, so that $l_{h}(0)=0$ and $\left|l_{h}^{\prime}\right| \leqq \frac{L}{h}$ by choice (4.63) of $r_{h}$;

- by (4.62a) and (4.62b), $\operatorname{sdist}\left(\cdot, U_{4} \cap \partial E_{h}\right) \rightarrow \operatorname{sdist}\left(\cdot, \partial U_{4}^{+}\right)$as $h \rightarrow+\infty$;

- by assumption $A_{k} \in \mathcal{A}_{m}$, the number of connected components of $\partial E_{h}$ lying strictly inside $U_{4}$ does not exceed $m$;

- by (g5), $\left|g_{1}-g_{0}\right| \leqq \phi\left(\mathbf{e}_{2}\right)$;

- by (4.2), $J_{u_{h}} \subset\left(\Omega_{h} \cap \partial E_{h}\right) \cup J_{E_{h}} \cup \widehat{L}_{h}$, where by (4.63), $\widehat{L}_{h}:=\sigma_{r_{h}}\left(U_{4 r_{h}} \cap L_{k_{h}}\right)$ satisfies $\mathcal{H}^{1}\left(\widehat{L}_{h}\right)<\frac{1}{h}$

- since

$$
\int_{U_{4}}\left|e\left(u_{h}\right)\right|^{2} \mathrm{~d} x \leqq \int_{U_{4 r_{h}}}\left|e\left(w_{k_{h}}\right)\right|^{2} \mathrm{~d} x \leqq \int_{B_{r_{0}}(0)}\left|e\left(w_{k_{h}}\right)\right|^{2} \mathrm{~d} x,
$$

by (4.2), we have sup $\left|e\left(u_{h}\right)\right|^{2} \mathrm{~d} x<\infty$;

- by (4.62d)-(4.62e),

$$
\lim _{h \rightarrow \infty} \int_{U_{1}}\left|\tau\left(u_{h}(x)\right)-\tau(u(x))\right| \mathrm{d} x=0,
$$

thus, possibly passing to further not relabelled subsequence, $u_{h} \rightarrow u=$ $u^{+} \chi_{U_{1}^{+}}+u^{-} \chi_{U_{1} \backslash U_{1}^{+}}$a.e. in $U_{1}$. 
This implies the claim.

Now we prove $(4.40 \mathrm{~g})$. Given $\delta \in(0,1)$, by the continuity of $\varphi,(\mathrm{g} 5)$ and $(\mathrm{g} 6)$, there exists $h_{\delta}^{1}>1$ such that

$$
\mu_{k_{h}}\left(\overline{U_{r_{h}}}\right) \geqq \widehat{\mu}_{k_{h}}\left(\overline{U_{r_{h}}}\right)-2 \delta \mathcal{H}^{1}\left(U_{r_{h}} \cap(\partial A \cup \Sigma)\right)
$$

for all $h>h_{\delta}^{1}$, where $\widehat{\mu}_{k}$ is defined exactly the same as $\mu_{k}$ with $\phi$ and $g_{s}$ in place of $\varphi$ and $g_{+}(x, s)$, here we used $(\mathrm{g} 5)$ as

$$
\int_{U_{r_{h}} \cap \Sigma}\left|g_{+}(x, s)-g_{+}(0, s)\right| \mathrm{d} \mathcal{H}^{1} \leqq \frac{\delta}{4} \mathcal{H}^{1}\left(U_{r_{h}} \cap \Sigma\right)
$$

provided that $h$ is large enough. By Lemma 4.7, there exists $h_{\delta}^{2}>h_{\delta}^{1}$ such that for any $h>h_{\delta}^{2}$,

$$
\frac{1}{r_{h}} \widehat{\mu}_{k_{h}}\left(\overline{U_{r_{h}}}\right) \geqq 2 \phi\left(\mathbf{e}_{2}\right)+2 g_{0}-2 \delta .
$$

Moreover, by (2.4) and non-negativity of $g_{+}$,

$$
\begin{aligned}
\mathcal{H}^{1}\left(U_{r_{h}} \cap(\partial A \cup \Sigma)\right. & =\mathcal{H}^{1}\left(U_{r_{h}} \cap \Omega \cap \partial A\right)+\mathcal{H}^{1}\left(U_{r_{h}} \cap \Sigma \cap \partial A\right) \\
& \leqq \frac{\mu_{k_{h}}\left(\overline{U_{r_{h}}}\right)}{c_{1}}+\mathcal{H}^{1}\left(U_{r_{h}} \cap \Sigma\right) .
\end{aligned}
$$

Thus, by (4.64) for any $h>h_{\delta}^{2}$,

$$
\left(1+\frac{\delta}{c_{1}}\right) \frac{\mu_{k_{h}}\left(\overline{U_{r_{h}}}\right)}{2 r_{h}}+2 \delta \frac{\mathcal{H}^{1}\left(U_{r_{h}} \cap \Sigma\right)}{2 r_{h}} \geqq \phi\left(\mathbf{e}_{2}\right)+g_{0}-\delta .
$$

From here and $(4.62 \mathrm{c})$ we get

$$
\phi\left(\mathbf{e}_{2}\right)+g_{0} \leqq \delta+\left(1+\frac{\delta}{c_{1}}\right)\left(\frac{\mu_{0}\left(U_{r_{h}}\right)}{2 r_{h}}+\frac{r_{h}}{2}\right)+2 \delta \frac{\mathcal{H}^{1}\left(U_{r_{h}} \cap \Sigma\right)}{2 r_{h}},
$$

therefore, first letting $h \rightarrow \infty$, then $\delta \rightarrow 0$, and using (g6), we obtain (4.40g).

Now we address the lower semicontinuity of $\mathcal{F}$. We start with the following auxiliary extension result:

Lemma 4.8. Let $P \subset \mathbb{R}^{n}$ and $Q \supset P$ be non-empty bounded connected Lipschitz open sets and let $\mathcal{E}: H^{1}\left(P ; \mathbb{R}^{2}\right) \rightarrow H^{1}\left(Q ; \mathbb{R}^{n}\right)$ be the Sobolev extension map, i.e., a bounded linear operator such that for any $v \in H^{1}\left(P ; \mathbb{R}^{n}\right), E v=v$ a.e. in $P$ and there exists $C_{P}>0$ such that $\|\mathcal{E} v\|_{H^{1}(Q)} \leqq C_{P}\|v\|_{H^{1}(P)}$. Consider any $\left\{u_{k}\right\} \subset H^{1}\left(P ; \mathbb{R}^{n}\right)$ such that

$$
\sup _{k} \int_{P}\left|e\left(u_{k}\right)\right|^{2} d x<\infty
$$

and $u_{k} \rightarrow$ u a.e. in $P$ for some function $u: P \rightarrow \mathbb{R}^{n}$. Then there exist a subsequence $\left\{u_{k_{l}}\right\}_{l}$ and $v \in H^{1}\left(Q ; \mathbb{R}^{n}\right)$ such that $v=u$ a.e. in $P$ and $\mathcal{E} u_{k_{l}} \rightarrow v$ in $L^{2}(Q)$ and

$$
\sup _{l}\left\|\mathcal{E} u_{k_{l}}\right\|_{H^{1}(Q)}<\infty
$$


Proof. By Proposition 3.7, $u \in H_{\mathrm{loc}}^{1}\left(P ; \mathbb{R}^{n}\right) \cap G S B D^{2}\left(P ; \mathbb{R}^{n}\right)$. By Poincaré-Korn inequality, there exist $c_{P}>0$ and a sequence $\left\{a_{k}\right\}$ of rigid displacements such that

$$
\left\|u_{k}+a_{k}\right\|_{H^{1}(P)} \leqq c_{P}\left\|e\left(u_{k}\right)\right\|_{L^{2}(P)}
$$

for any $k$. Since $u_{k} \rightarrow u$ a.e. in $P$, reasoning as in the proof of Proposition 3.7 (with $P$ in place of $B_{\epsilon}$ ), up to a not relabelled subsequence, $a_{k} \rightarrow a$ a.e. in $\mathbb{R}^{n}$ for some rigid displacement $a: \mathbb{R}^{n} \rightarrow \mathbb{R}^{n}$. In particular, $H^{1}\left(P ; \mathbb{R}^{n}\right)$-norm of $a_{k}$ is uniformly bounded independently of $k$, hence,

$$
\sup _{k}\left\|\mathcal{E} a_{k}\right\|_{H^{1}(Q)}<\infty
$$

Since

$$
\left\|\mathcal{E}\left(u_{k}+a_{k}\right)\right\|_{H^{1}(Q)} \leqq C_{P}\left\|u_{k}+a_{k}\right\|_{H^{1}(P)} \leqq C_{P} c_{P}\left\|e\left(u_{k}\right)\right\|_{L^{2}(P)},
$$

by (4.65), the linearity of $E$ and (4.67),

$$
\sup _{k}\left\|\mathcal{E} u_{k}\right\|_{H^{1}(Q)}<\infty .
$$

Thus, by the Rellich-Kondrachov Theorem, there exists a not relabelled subsequence $\left\{u_{k}\right\}$ and $v \in H^{1}\left(Q ; \mathbb{R}^{n}\right)$ such that $\mathcal{E} u_{k} \rightarrow v$ in $L^{2}(Q)$ and a.e. in $Q$.

Proof of Theorems 2.8. Without loss of generality, we assume that

$$
\sup _{k \geqq 1} \int_{A_{k} \cup S}\left|e\left(u_{k}\right)\right|^{2} \mathrm{~d} x+\mathcal{H}^{1}\left(\partial A_{k}\right)<\infty,
$$

The lower semicontinuity of the elastic-energy part can be shown by using convexity $W(x, \cdot)$. Indeed, let $D \subset \subset \operatorname{Int}(A)$. Then by $\tau_{\mathcal{A}}$-convergence of $A_{k}, D \subset \subset \operatorname{Int}\left(A_{k}\right)$ for all large $k$. Since $u_{k} \rightarrow u$ a.e. in $A \cup S$, by (4.69) and the weak-compactness of $L^{2}(D \cup S), e\left(u_{k}\right) \rightarrow e(u)$ in $L^{2}(D \cup S)$. Therefore, from the convexity of $\mathcal{W}(D, \cdot)$ it follows that

$$
\mathcal{W}(D, u) \leqq \liminf _{k \rightarrow \infty} \mathcal{W}\left(D, u_{k}\right) \leqq \liminf _{k \rightarrow \infty} \mathcal{W}\left(A_{k}, u_{k}\right)
$$

Now letting $D \nearrow A \cup S$ we get

$$
\mathcal{W}(A, u) \leqq \liminf _{k \rightarrow \infty} \mathcal{W}\left(A_{k}, u_{k}\right)
$$

Since $\mathcal{S}(E, v)=\mathcal{S}\left(E, J_{v} ; \varphi, g\right)$ with $J_{E}=J_{v}$ and $g(x, s)=\beta(x) s$, the lower semicontinuity of of the surface part, follows from Proposition 4.1 provided that for $\mathcal{H}^{1}$-a.e. $x \in J_{u}$ there exists $r_{x}>0, w_{k} \in G S B D\left(B_{r_{x}}(x) ; \mathbb{R}^{2}\right)$ and relatively open sets $L_{k}$ of $\Sigma$ with $\mathcal{H}^{1}\left(L_{k}\right)<1 / k$ such that (4.2) holds. Let

$$
r_{0}^{x}:=\frac{1}{4} \min \{\operatorname{dist}(x, \partial \Omega \backslash \Sigma), \operatorname{dist}(x, \partial S \backslash \Sigma)\}
$$


so that $B_{r_{0}^{x}}(x) \subset \subset \Omega \cup \Sigma \cup S$, and choose $r=r_{x} \in\left(0, r_{0}^{x}\right)$ such that

$$
\begin{aligned}
\mathcal{H}^{1}\left(\partial B_{r}(x) \cap \partial A_{k}\right) & =\mathcal{H}^{1}\left(\partial B_{r}(x) \cap J_{u_{k}}\right) \\
& =\mathcal{H}^{1}\left(\partial B_{r}(x) \cap \partial A\right)=\mathcal{H}^{1}\left(\partial B_{r}(x) \cap J_{u}\right)=0
\end{aligned}
$$

(see [46, Proposition 2.6]) and $B_{r}(x) \cap S$ is connected. We construct $\left\{w_{k}\right\}$ by extending $\left\{u_{k}\right\}$ in $B_{r}(x) \backslash\left(A_{k} \cup S\right)$ without creating extra jumps at the interface on the exposed surface of the substrate. More precisely, we apply Lemma 4.8 with $Q:=B_{r}(x), P:=B_{r}(x) \cap S$, and $\left.u_{k}\right|_{P}$. Since $u_{k} \rightarrow u$ a.e. in $P$, by Lemma 4.8, there exist $v \in H^{1}\left(Q ; \mathbb{R}^{2}\right)$ and a not relabelled subsequence $\left\{u_{k}\right\}$ such that the Sobolev extension $\mathcal{E} u_{k}$ of $\left.u_{k}\right|_{P}$ to $Q$ converges to $v$ a.e. in $Q$. Define

$$
w_{k}:=u_{k} \chi_{B_{r}(x) \cap\left(A_{k} \cup S\right)}+\mathcal{E} u_{k} \chi_{B_{r}(x) \backslash\left(A_{k} \cup S\right)} .
$$

Perturbing $w_{k}$ slightly if necessary, we can assume $J_{w_{k}}=\Gamma:=B_{r} \cap\left(J_{u_{k}} \cup(\Omega \cup\right.$ $\left.\left.\partial^{*} A_{k}\right) \cup\left(A_{k}^{(1)} \cap \partial A_{k}\right)\right)$ up to a $\mathcal{H}^{1}$-negligible set. In fact, by [46, Proposition 2.6] there exist $\xi \in \mathbb{R}^{2}$ with arbitrarily small $|\xi|>0$ for which $\mathcal{H}^{1}\left(\left\{y \in \Gamma:\left[u_{k}\right](y)=\right.\right.$ $\xi\})=0$ (with $\left[u_{k}\right](x)$ the size of the jump of $u_{k}$ ), and hence, we can perturb $u_{k}$ with a $W^{1, \infty}\left(B_{r}(x) \backslash \Gamma\right)$-function with arbitrarily small norm, which is equal to $\xi$ on an arbitrarily large subset of $\Gamma$. By construction,

$$
w_{k} \rightarrow w:=u \chi_{B_{r}(x) \cap(A \cup S)}+v \chi_{B_{r}(x) \cap(A \cup S)},
$$

thus, by [15, Theorem 1.1], $w \in G S B D^{2}\left(B_{r}(x) ; \mathbb{R}^{2}\right)$. Notice also that $J_{u} \subset J_{w}$ since $w=u$ a.e. in $B_{r}(x) \cap(A \cup S)$. Thus $w_{k}$ and $w$ satisfy (4.2).

We conclude this section by proving a lower semicontinuity property of $\mathcal{F}^{\prime}$ with respect to $\tau_{\mathcal{C}}^{\prime}$. Observe that if $\left(A_{k}, u_{k}\right) \stackrel{\tau_{\mathcal{C}}^{\prime}}{\rightarrow}(A, u)$, then $A=\operatorname{Int}(A)$ so that the weak convergence of $u_{k}$ to $u$ in $H_{\text {loc }}^{1}\left(A \cup S ; \mathbb{R}^{2}\right)$ is well-defined. However, notice that $\mathcal{C}_{m}^{\prime}$ is not closed with respect to $\tau_{\mathcal{C}}^{\prime}$-convergence.

Proposition 4.9. (Lower semicontinuity of $\left.\mathcal{F}^{\prime}\right)$ Assume $(\mathrm{H} 1)-(\mathrm{H} 3)$. If $\left(A_{k}, u_{k}\right) \in$ $\mathcal{C}_{m}^{\prime}$ and $(A, u) \in \mathcal{C}$ are such that $\left(A_{k}, u_{k}\right) \stackrel{\tau_{\mathcal{C}}^{\prime}}{\rightarrow}(A, u)$, then

$$
\liminf _{k \rightarrow \infty} \mathcal{F}^{\prime}\left(A_{k}, u_{k}\right) \geqq \mathcal{F}^{\prime}(A, u) .
$$

Proof. Consider the auxiliary functional $\tilde{\mathcal{F}}: \mathcal{C} \rightarrow \mathbb{R}$ defined as

$$
\widetilde{\mathcal{F}}(A, u)=\mathcal{F}(A, u)-\int_{\Sigma \cap A^{(0)} \cap \partial A}\left(\phi\left(x, v_{A}\right)+\beta\right) \mathrm{d} \mathcal{H}^{1} .
$$

Since $\widetilde{\mathcal{F}}$ does not see wetting layer energy,

$$
\mathcal{F}^{\prime}(G, u)=\widetilde{\mathcal{F}}(G, u)-\int_{\Sigma} \beta \mathrm{d} \mathcal{H}^{1}=\mathcal{F}(G \cup \Sigma, u)-\int_{\Sigma} \beta \mathrm{d} \mathcal{H}^{1}
$$

for any $G \in \mathcal{A}_{m}^{\prime}:=\left\{A \in \underset{\mathcal{A}}{\mathcal{F}}: A \cup \Sigma \in \mathcal{A}_{m}\right\}$. Repeating the proof of Theorem 2.8 one can readily show that $\widetilde{\mathcal{F}}$ is also $\tau_{\mathcal{C}}$-lower semicontinuous. 
Now we prove (4.70). Without loss of generality we suppose that liminf is a finite limit. Let $E_{k}:=A_{k} \cup \Sigma$. By the definition of $\mathcal{A}_{m}^{\prime}$ and $\tau_{\mathcal{C}}^{\prime}$-convergence, $\left\{E_{k}\right\} \subset \mathcal{A}_{m}$ and $\sup \mathcal{H}^{1}\left(\partial E_{k}\right)<\infty$, therefore by Proposition 3.3, there exist a (not relabelled) subsequence and $E \in \mathcal{A}_{m}$ such that $E_{k} \stackrel{{ }^{\tau_{\mathcal{C}}}}{\rightarrow} E$. By Remark 2.2, $A=\operatorname{Int}(E)$, thus, by (4.71),

$$
\begin{aligned}
\lim _{k \rightarrow \infty} \mathcal{F}^{\prime}\left(A_{k}, u_{k}\right) & =\lim _{k \rightarrow \infty} \tilde{\mathcal{F}}\left(A_{k} \cup \Sigma, u_{k}\right)-\int_{\Sigma} \beta \mathrm{d} \mathcal{H}^{1} \\
& \geqq \widetilde{\mathcal{F}}(E, u)-\int_{\Sigma} \beta \mathrm{d} \mathcal{H}^{1} \geqq \mathcal{F}^{\prime}(A, u) .
\end{aligned}
$$

\section{Existence}

In this section we prove Theorems 2.6 and 2.9.

Proof of Theorem 2.6. We start by showing the existence of solutions of problems (CP) and (UP).

For the constrained minimum problem, let $\left\{\left(A_{k}, u_{k}\right)\right\} \subset \mathcal{C}_{m}$ be arbitrary minimizing sequence such that

$$
\sup _{k \geqq 1} \mathcal{F}\left(A_{k}, u_{k}\right)<\infty .
$$

By Theorem 2.7, there exist $(A, u) \in \mathcal{C}_{m}$, a not relabelled subsequence $\left\{\left(A_{k}, u_{k}\right)\right\}$ and a sequence $\left\{D_{k}, v_{k}\right\} \subset \mathcal{C}_{m}$ such that $\left(D_{k}, v_{k}\right) \stackrel{\tau_{\mathcal{C}}}{\rightarrow}(A, u)$ and $\left|B_{k}\right|=\left|A_{k}\right|=\mathrm{v}$ and

$$
\liminf _{k \rightarrow \infty} \mathcal{F}\left(A_{k}, u_{k}\right) \geqq \liminf _{k \rightarrow \infty} \mathcal{F}\left(D_{k}, v_{k}\right) .
$$

By Lemma 3.2 (b), $D_{n} \rightarrow A$ in $L^{1}\left(\mathbb{R}^{2}\right)$ so that $|A|=\mathrm{v}$. Now by Theorem 2.8

$$
\inf _{(V, v) \in \mathcal{C}_{m},|V|=\mathrm{v}} \mathcal{F}(V, v)=\liminf _{k \rightarrow \infty} \mathcal{F}\left(D_{k}, v_{k}\right) \geqq \mathcal{F}(A, u)
$$

so that $(A, u)$ is a minimizer. The case of the unconstrained problem is analogous.

Now we prove (2.7). Observe that in general

$$
\inf _{(A, u) \in \mathcal{C}} \mathcal{F}^{\lambda}(A, u) \leqq \inf _{(A, u) \in \mathcal{C},|A|=\mathrm{v}} \mathcal{F}(A, u)
$$

and the same inequality still holds if we replace $\mathcal{C}$ with $\mathcal{C}_{m}$. Moreover, any solution $(A, u) \in \mathcal{C}_{m}$ of (UP) satisfying $|A|=\mathrm{v}$ solves also (CP). By Proposition A.6, there exists a universal constant $\lambda_{0}>0$ with the following property: $(A, u) \in \mathcal{C}_{m}$ is a solution of (CP) if and only if it solves (UP) for some (and hence for all) $\lambda \geqq \lambda_{0}$. Thus,

$$
\inf _{(A, u) \in \mathcal{C}_{m}} \mathcal{F}^{\lambda}(A, u)=\inf _{(A, u) \in \mathcal{C}_{m},|A|=\mathrm{v}} \mathcal{F}(A, u)
$$


for any $m \geqq 1$ and $\lambda \geqq \lambda_{0}$. Since $\mathcal{C}_{m} \subset \mathcal{C}_{m+1} \subset \mathcal{C}$, the map

$$
m \in \mathbb{N} \mapsto \inf _{(A, u) \in \mathcal{C}_{m},|A|=\mathrm{v}} \mathcal{F}(A, u)
$$

is nonincreasing, and

$$
\inf _{(A, u) \in \mathcal{C},|A|=\mathrm{v}} \mathcal{F}(A, u) \leqq \inf _{(A, u) \in \mathcal{C}_{m},|A|=\mathrm{v}} \mathcal{F}(A, u),
$$

so that

$$
\inf _{(A, u) \in \mathcal{C},|A|=\mathrm{v}} \mathcal{F}(A, u) \leqq \lim _{m \rightarrow \infty} \inf _{(A, u) \in \mathcal{C}_{m},|A|=\mathrm{v}} \mathcal{F}(A, u) .
$$

In view of (5.1) and (5.3) to conclude the proof of (2.7) it suffices to show that for any $\epsilon \in(0,1)$ and $\lambda>\lambda_{0}$, there exist $n \geqq 1$ and $(E, v) \in \mathcal{C}_{n}$ such that

$$
\inf _{(A, u) \in \mathcal{C}} \mathcal{F}^{\lambda}(A, u)+\epsilon \geqq \mathcal{F}^{\lambda}(E, v)
$$

Indeed, by (5.4) and (5.2), given $\epsilon \in(0,1)$

$$
\begin{aligned}
\inf _{(A, u) \in \mathcal{C}} \mathcal{F}^{\lambda}(A, u)+\epsilon & \geqq \mathcal{F}^{\lambda}(E, v) \\
\geqq & \inf _{(A, u) \in \mathcal{C}_{n}} \mathcal{F}^{\lambda}(A, u) \\
& =\inf _{(A, u) \in \mathcal{C}_{n},|A|=\mathrm{v}} \mathcal{F}(A, u) \\
& \geqq \lim _{m \rightarrow \infty} \inf _{(A, u) \in \mathcal{C}_{m},|A|=\mathrm{v}} \mathcal{F}(A, u) .
\end{aligned}
$$

Now letting $\epsilon \rightarrow 0$ and using (5.2) and (5.3) we get (2.7).

We construct $(E, v) \in \mathcal{C}_{n}$ satisfying (5.4) as follows. Fix $\epsilon \in(0,1)$ and $\lambda>\lambda_{0}$, and choose $(A, u) \in \mathcal{C}$ such that

$$
\inf \mathcal{F}^{\lambda}+\frac{\epsilon}{4}>\mathcal{F}^{\lambda}(A, u)
$$

Notice that:

- removing the exterior filaments decreases the energy, i.e., $\mathcal{F}(A, u) \geqq$ $\mathcal{F}(\operatorname{Int}(A), u)$, thus, we assume that $A=\operatorname{Int}(A)$ so that $A$ is open;

- let $\left\{A_{i}\right\}_{i \in I}$ be all open connected components of $A$. Since

$$
\mathcal{F}(A, u)=\sum_{i \in I} \mathcal{F}\left(A_{j}, u\right)
$$

by the finiteness of $\mathcal{F}(A, u)$ and $|A|$, we can choose a finite set $I^{\prime} \subset I$ such that

$$
|A| \leqq \sum_{j \in I^{\prime}}\left|A_{j}\right|+\frac{\epsilon}{8 \lambda} .
$$

Thus, setting $A^{\prime}:=\bigcup_{j \in I^{\prime}} A_{j}$ and $u^{\prime}:=\left.u\right|_{A^{\prime}}$ we get that $\left(A^{\prime}, u^{\prime}\right) \in \mathcal{C}$ and

$$
\mathcal{F}^{\lambda}(A, u)+\frac{\epsilon}{8} \geqq \mathcal{F}\left(A^{\prime}, u^{\prime}\right)
$$


- let $\left\{F_{j}\right\}_{j \in J}$ be all open connected components of $\Omega \backslash \overline{A^{\prime}}$. Since $\partial F_{j} \subset \partial A^{\prime} \cup \partial \Omega$ and $|\Omega|+\mathcal{H}^{1}\left(\partial A^{\prime}\right)+\mathcal{H}^{1}(\partial \Omega)<\infty$, there exists a finite set $J^{\prime} \subset J$ such that

$$
\sum_{j \in J \backslash J^{\prime}} \mathcal{S}\left(F_{j} ; u_{0}\right)<\frac{\epsilon}{16}
$$

and

$$
\sum_{j \in J \backslash J^{\prime}}\left|F_{j}\right|<\frac{\epsilon}{16 \lambda} .
$$

Hence, setting $A^{\prime \prime}:=A \cup \bigcup_{j \in J \backslash J^{\prime}} F_{j}$ and $u^{\prime \prime}:=u^{\prime} \chi_{A^{\prime}}+u_{0} \chi \bigcup_{j \in J \backslash J^{\prime}} F_{j}$ we get that $\left(A^{\prime}, u^{\prime}\right) \in \mathcal{C}$ and

$$
\mathcal{F}^{\lambda}\left(A^{\prime}, u^{\prime}\right)+\frac{\epsilon}{4} \geqq \mathcal{F}^{\lambda}\left(A^{\prime \prime}, u^{\prime \prime}\right) .
$$

Notice that for $j \in J \backslash J^{\prime}$, the set $\partial A^{\prime} \cap \partial F_{j}$ becomes the internal crack for $A^{\prime \prime}$, and there is no elastic energy contribution in $F_{j}$;

see Fig. 2.

Hence, $A^{\prime \prime}$ is a union of finitely many connected open sets with finitely many "holes" inside so that $\partial \overline{A^{\prime \prime}}=\overline{\partial^{*} A^{\prime \prime}}$ consists of finitely many connected sets with finite length. Moreover, by (5.5), (5.6) and (5.7),

$$
\inf \mathcal{F}^{\lambda}+\frac{\epsilon}{2} \geqq \mathcal{F}^{\lambda}\left(A^{\prime \prime}, u^{\prime \prime}\right) .
$$

In view of (5.5) and (5.8) it remains to show that there exists $m \geqq 1$ and $(E, v) \in \mathcal{C}_{m}$ such that

$$
\mathcal{F}^{\lambda}\left(A^{\prime \prime}, u^{\prime \prime}\right)+\frac{\epsilon}{2}>\mathcal{F}^{\lambda}(E, v) .
$$

Let $G:=\operatorname{Int}\left(\overline{A^{\prime \prime}}\right)$ so that $G$ is open and $\partial G=\overline{\partial^{*} G}$. Since $\Sigma$ is a 1-dimensional Lipschitz manifold, by the outer regularity of $\mathcal{H}^{1} L \Sigma$ there exists a finite union $I$ of subintervals of $\Sigma$ such that

$$
J_{u^{\prime \prime}} \subseteq I
$$

and

$$
\mathcal{H}^{1}\left(I \backslash J_{u^{\prime \prime}}\right)<\frac{\epsilon}{64 c_{2}} .
$$

Since $\partial \Omega$ is Lipschitz and $\varphi$, there exists a Lipschitz open set $V \subset \Omega$ such that $\partial V \cap \Sigma=I$ and

$$
\int_{\Omega \cap \partial^{*} V} \varphi\left(x, v_{U}\right) \mathrm{d} \mathcal{H}^{1} \leqq \int_{I} \varphi\left(x, v_{\Sigma}\right) \mathrm{d} \mathcal{H}^{1}+\frac{\epsilon}{64}
$$

and

$$
|V|<\frac{\epsilon}{32 \lambda}
$$




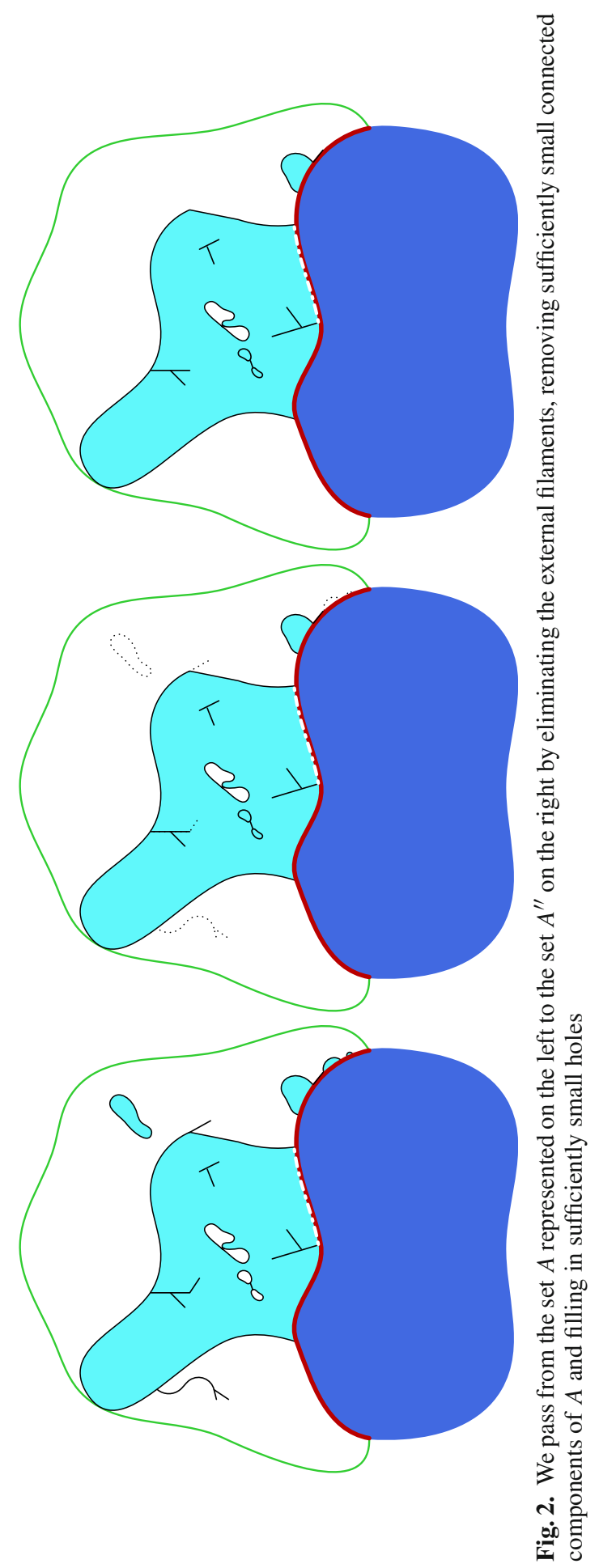


since $\varphi$ is uniformly continuous, basically, $V$ is obtained slightly translating $I$ inside A.

Let us consider $(B, w) \in \mathcal{C}$ with $B:=A^{\prime \prime} \backslash V$ and $w=u^{\prime \prime} \chi_{A^{\prime \prime} \backslash V}$. Since $B \subset A^{\prime \prime}$,

$$
\mathcal{W}(B, w) \leqq \mathcal{W}\left(A^{\prime \prime}, u^{\prime \prime}\right)
$$

since $J_{w}=\emptyset$, by (5.10) and (5.11)

$$
\mathcal{S}(B, w) \leqq \mathcal{S}\left(A^{\prime \prime}, u^{\prime \prime}\right)+\frac{\epsilon}{16},
$$

and by (5.12)

$$
\lambda|| B|-\mathrm{v}| \leqq \lambda|| A^{\prime \prime}|-\mathrm{v}|+\frac{\epsilon}{16}
$$

Thus,

$$
\mathcal{F}^{\lambda}(B, w) \leqq \mathcal{F}^{\lambda}\left(A^{\prime \prime}, u^{\prime \prime}\right)+\frac{\epsilon}{8}
$$

Let $\widetilde{w} \in G S B D^{2}\left(\operatorname{Int}(\overline{B \cup S}) ; \mathbb{R}^{2}\right)$ be such that $\widetilde{w}=w$ a.e. in $\overline{B \cup S}$. Notice that $\Sigma \cap J_{\widetilde{w}}=\varnothing$ and $J_{\widetilde{w}} \subseteq B^{(1)} \cap \partial B$. Perturbing approximate continuity points of $w$ along $B^{(1)} \cap \partial B$ (as has been done in the proof of Theorem 2.8), we may suppose that $B^{(1)} \cap \partial B$ is a jump set for $\widetilde{w}$. Hence, using the Vitali class of covering squares for $J_{\widetilde{w}}$ contained in $\Omega$ in the proof of [14, Theorem 1.1] we find $\left.\widetilde{v} \in S B V^{2}(\operatorname{Int}(B) \cup S \cup \Sigma) ; \mathbb{R}^{2}\right) \cap W^{1, \infty}\left(\operatorname{Int}(B \cup S \cup \Sigma) ; \mathbb{R}^{2}\right)$ such that $J_{\widetilde{v}}$ is contained in a union of finitely many closed connected curves in $\bar{B}$ (see [14, pp. 1353 and 1359]) and

$$
\begin{aligned}
& \int_{B \cup S}|e(\widetilde{v})-e(\widetilde{w})|^{2} \mathrm{~d} x<\frac{\epsilon^{2}}{512(\mathcal{W}(B, \widetilde{w})+1)\left(\|\mathbb{C}\|_{\infty}+1\right)}, \\
& \mathcal{H}^{1}\left(J_{\widetilde{v}} \Delta J_{\widetilde{w}}\right)<\frac{\epsilon}{32 c_{2}} .
\end{aligned}
$$

Notice that we do not need to control the boundary trace of $\widetilde{w}$ that's why we can use the approximation result [14, Theorem 1.1] only inside $B \cup \Sigma \cup S$. Moreover, since $J_{\widetilde{w}} \subset \operatorname{Int}(\bar{B})$ and we use Vitali class of covering cubes only inside $\Omega$ by the formula [14, page 1359] for the jump of the approximating sequence, it follows that $J_{\widetilde{v}} \subset \bar{B}$. In particular, $\widetilde{v} \in H^{1}\left(S ; \mathbb{R}^{2}\right)$.

By the convexity of the elastic energy and the Cauchy-Schwarz inequality for nonnegative quadratic forms,

$$
\begin{aligned}
& \int_{B \cup S} W\left(x, e(\widetilde{v})-E_{0}\right) \mathrm{d} x \leqq \int_{B \cup S} W\left(x, e(\widetilde{w})-E_{0}\right) \mathrm{d} x \\
& \quad+2 \int_{B \cup S} \mathbb{C}(x)\left[e(\widetilde{v})-E_{0}\right]:[e(\widetilde{v})-e(\widetilde{w})] \mathrm{d} x \\
& \quad \leqq \int_{B \cup S} W\left(x, e(\widetilde{w})-E_{0}\right) \mathrm{d} x
\end{aligned}
$$




$$
+2 \sqrt{\int_{B \cup S} W\left(x, e(\widetilde{v})-E_{0}\right) \mathrm{d} x} \sqrt{\int_{B \cup S} W(x, e(\tilde{v})-e(\widetilde{w})) \mathrm{d} x} .
$$

Since

$$
\int_{B \cup S} W(x, e(\widetilde{v})-e(\widetilde{w})) \mathrm{d} x \leqq\|\mathbb{C}\|_{\infty} \int_{B \cup S}|e(\widetilde{v})-e(\widetilde{w})|^{2} \mathrm{~d} x,
$$

and

$$
\begin{aligned}
& \int_{B \cup S} W\left(x, e(\widetilde{v})-E_{0}\right) \mathrm{d} x \\
& \quad \leqq 2 \int_{B \cup S} W(x, e(\widetilde{v})-e(\widetilde{w})) \mathrm{d} x+2 \int_{B \cup S} W\left(x, e(\widetilde{w})-E_{0}\right) \mathrm{d} x \\
& \quad \leqq 2\|\mathbb{C}\|_{\infty} \int_{B \cup S}|e(\widetilde{v})-e(\widetilde{w})|^{2} \mathrm{~d} x+2 \mathcal{W}(B, \widetilde{w}) \leqq 2 \mathcal{W}(B, \widetilde{w})+2,
\end{aligned}
$$

by (5.15a) and (5.16),

$$
\int_{B \cup S} W\left(x, e(\widetilde{v})-E_{0}\right) \mathrm{d} x \leqq \int_{B \cup S} W\left(x, e(\widetilde{w})-E_{0}\right) \mathrm{d} x+\frac{\epsilon}{4} .
$$

As $J_{v}$ is contained in at most finitely many closed $C^{1}$-curves, we can find finitely many arcs of those curves whose union $\Gamma \subset \bar{B}$ still contains $J_{\widetilde{v}}$ and satisfies

$$
\mathcal{H}^{1}\left(\Gamma \backslash J_{\widetilde{v}}\right)<\frac{\epsilon}{32 c_{2}} .
$$

Set $E:=\operatorname{Int}(\bar{B}) \backslash \Gamma$ and $v:=\left.\widetilde{v}\right|_{E}$. We show that $(E, v)$ satisfies (5.9). Note that $J_{v} \cap(E \cup S)=\emptyset$, thus, $v \in H_{\text {loc }}^{1}\left(E \cup S ; \mathbb{R}^{2}\right) \cap G S B D^{2}\left(\operatorname{Int}(E \cup S \cup \Sigma) ; \mathbb{R}^{2}\right)$. Moreover, by construction, $\overline{\partial^{*} A^{\prime \prime}}, \Gamma$ and $\partial V$ consist of finitely many connected components, therefore, there exists $m \geqq 1$ such that $(E, v) \in \mathcal{C}_{m}$. Notice that by the definition of $E$,

$$
|E|=|\operatorname{Int}(\bar{B})|=|B|,
$$

by the definition of $v, \widetilde{w}$ and (5.17),

$$
\mathcal{W}(E, v) \leqq \mathcal{W}(B, \widetilde{w})+\frac{\epsilon}{4}=\mathcal{W}(B, w)+\frac{\epsilon}{4},
$$

and by $\partial^{*} E=\partial^{*} B,(5.18)$ and (5.15b) as well as (2.4) and (5.13),

$$
\mathcal{S}(E, v) \leqq \mathcal{S}(B, w)+\frac{\epsilon}{8}
$$

From (5.19)-(5.21) we get

$$
\mathcal{F}^{\lambda}(E, v) \leqq \mathcal{F}^{\lambda}(B, w)+\frac{3 \epsilon}{8} .
$$

Combining this with (5.14) we obtain (5.9). 
Proof of Theorem 2.9. In view of Proposition 4.9 the assertion follows from the direct methods of the Calculus of Variations.

Acknowledgements. Open access funding provided by Austrian Science Fund (FWF). Sh. Kholmatov acknowledges support from the Austrian Science Fund (FWF) project M 2571N32. P. Piovano acknowledges support from the Vienna Science and Technology Fund (WWTF), the City of Vienna, and Berndorf Privatstiftung through Project MA16-005, from the Austrian Science Fund (FWF) through project P 29681, and from BMBWF through the OeAD-WTZ project HR 08/2020. Moreover, the authors are grateful to the anonymous referees for their careful reading and valuable comments.

Open Access This article is licensed under a Creative Commons Attribution 4.0 International License, which permits use, sharing, adaptation, distribution and reproduction in any medium or format, as long as you give appropriate credit to the original author(s) and the source, provide a link to the Creative Commons licence, and indicate if changes were made. The images or other third party material in this article are included in the article's Creative Commons licence, unless indicated otherwise in a credit line to the material. If material is not included in the article's Creative Commons licence and your intended use is not permitted by statutory regulation or exceeds the permitted use, you will need to obtain permission directly from the copyright holder. To view a copy of this licence, visit http://creativecommons.org/ licenses/by/4.0/.

Publisher's Note Springer Nature remains neutral with regard to jurisdictional claims in published maps and institutional affiliations.

\section{Appendix A}

In this section we recall some results from the literature for the reader's convenience.

\section{A.1. Kuratowski Convergence}

Let $\left\{E_{k}\right\}$ be a sequence of subsets of $\mathbb{R}^{2}$. A set $E \subset \mathbb{R}^{2}$ is the $\mathcal{K}$-lower limit of $\left\{E_{k}\right\}$ if for every $x \in E$ and $\rho>0$ there exists $n>0$ such that $B_{\rho}(x) \cap E_{k} \neq \emptyset$ for all $k>n$. A set $E \subset \mathbb{R}^{2}$ is the $\mathcal{K}$-upper limit of $\left\{E_{k}\right\}$ if for every $x \in E$ and $\rho>0$ and $n \in \mathbb{N}$ there exists $k>n$ such that $B_{\rho}(x) \cap E_{k} \neq \emptyset$.

The $\mathcal{K}$-lower and $\mathcal{K}$-upper limits of $\left\{E_{k}\right\}$ are always exist and respectively denoted as

$$
\mathcal{K}-\liminf _{k \rightarrow \infty} E_{k} \quad \text { and } \quad \mathcal{K}-\limsup _{k \rightarrow \infty} E_{k}
$$

It is clear that both sets are closed sets and

$$
\mathcal{K}-\liminf _{k \rightarrow \infty} E_{k} \subseteq \mathcal{K}-\limsup _{k \rightarrow \infty} E_{k}
$$

in case of equality, we say $E_{k}$ converges to $E=\mathcal{K}-\liminf _{k \rightarrow \infty} E_{k}=\mathcal{K}-\limsup _{k \rightarrow \infty} E_{k}$ in the Kuratowski sense and write

$$
E=\mathcal{K}-\lim _{k \rightarrow \infty} E_{k} \quad \text { or } \quad E_{k} \stackrel{\mathcal{K}}{\rightarrow} E .
$$

Observe that by the definition of $\mathcal{K}$-convergence, $E_{k}$ and $\overline{E_{k}}$ have the same $\mathcal{K}$-upper and $\mathcal{K}$-lower limits. Moreover, Kuratowski limit is always unique. 
Proposition A.1. The following assertions are equivalent:

(a) $E_{k} \stackrel{\mathcal{K}}{\rightarrow} E$;

(b) if $x_{k} \in E_{k}$ converges to some $x \in \mathbb{R}^{2}$, then $x \in E$, and for every $x \in E$ there exists a subsequence $x_{n_{k}} \in E_{n_{k}}$ converging to $x$;

(c) $\operatorname{dist}\left(\cdot, E_{k}\right) \rightarrow \operatorname{dist}(\cdot, E)$ locally uniformly in $\mathbb{R}^{2}$;

(d) if, in addition, $\left\{E_{k}\right\}$ is uniformly bounded, $E_{k} \rightarrow E$ with respect to Hausdorff distance dist $_{\mathcal{H}}$, where

$$
\operatorname{dist}_{\mathcal{H}}(A, B):=\left\{\begin{array}{lc}
0 & \text { if } A=B=\emptyset, \\
\max \left\{\sup _{x \in A} \operatorname{dist}(x, B), \sup _{x \in B} \operatorname{dist}(x, A)\right\} & \text { otherwise. }
\end{array}\right.
$$

\section{A.2. Rectifiability in $\mathbb{R}^{2}$}

Below we recall some important regularity properties of compact connected subsets of finite $\mathcal{H}^{1}$-measure of $\mathbb{R}^{2}$ most of them are taken from [29, Chapters 2 and 3].

The image $\Gamma$ of a continuous injection $\gamma:[a, b] \rightarrow \mathbb{R}^{2}$ is called curve (or Jordan curve), and $\gamma$ is the parametrization of $\Gamma$. Clearly, any curve is compact and connected set, hence it is $\mathcal{H}^{1}$-measurable. The length of a curve $\Gamma$ is defined as

$$
\sup s(\gamma, P),
$$

where $P=\left\{t_{0}, t_{1}, \ldots, t_{N}\right\}$ is a partition of $[a, b]$, i.e., $a=t_{0}<t_{1}<\ldots<t_{N}=b$,

$$
s(\gamma, P):=\sum_{i=1}^{N}\left|\gamma\left(t_{i-1}\right)-\gamma\left(t_{i}\right)\right|,
$$

and sup is taken over all partitions $P$ of $[a, b]$. By [29, Lemma 3.2], the length of curve $\Gamma$ is equal to $\mathcal{H}^{1}(\Gamma)$.

Any curve $\Gamma$ with finite length admits so-called arclength parametrization in $\left[0, \mathcal{H}^{1}(\Gamma)\right]$, which is a Lipschitz parametrization $\gamma_{o}$ with Lipschitz constant 1 . Hence, by the Rademacher Theorem [3, Theorem 2.14] it is differentiable at a.e. $\ell \in\left(0, \mathcal{H}^{1}(\Gamma)\right)$ and $\left|\dot{\gamma}_{o}(\ell)\right| \leqq 1$. Hence $\Gamma$ has an (approximate) tangent line at $\mathcal{H}^{1}$-a.e. $x \in \Gamma$ and we can define the approximate unit normal $v_{\Gamma}(x)$ of $\Gamma$ at $\mathcal{H}^{1}$-a.e. $x \in \Gamma$.

We recall the following characteristics of compact connected $\mathcal{H}^{1}$-rectifiable sets (see [29, Theorem 3.14] and [36, Section 2]).

Proposition A.2. (Rectifiable connected sets) Every connected compact set $K \subset \bar{U}$ with $\mathcal{H}^{1}(K)<\infty$ is arcwise connected and countably $\mathcal{H}^{1}$-rectifiable, i.e,

$$
K:=N \cup \bigcup_{j \geqq 1} \Gamma_{j},
$$

where $N$ is a $\mathcal{H}^{1}$-negligible set and $\Gamma_{j}:=\gamma_{j}([0,1])$ is a curve with finite length for a parametrization $\gamma_{j}:[0,1] \rightarrow \mathbb{R}^{2}$ such that

$$
\gamma_{j}((0,1)) \cap \bigcup_{i=1}^{j-1} \Gamma_{i}=\emptyset, \quad j \geqq 2 .
$$


Remark A.3. (Rectifiable curve is locally Jordan) Let $\Gamma$ be a rectifiable curve. Then for $\mathcal{H}^{1}$ a.e. $x \in \Gamma$ there exists $r_{x}>0$ such that $B_{r_{x}}(x) \backslash \Gamma$ has exactly two connected components. Indeed, suppose that there exists $x \in \Gamma$ such that $\theta^{*}(\Gamma, x)=\theta_{*}(\Gamma, x)=1$ and $B_{r}(x) \backslash \Gamma$ has at least three connected components for every $r>0$ such that endpoints of $\Gamma$ lie outside $\overline{B_{r}(x)}$. Then $\left(B_{r}(x) \backslash B_{r / 2}(x)\right) \cap \Gamma$ should have three connected components and as a result $\mathcal{H}^{1}\left(\left(B_{r}(x) \backslash B_{r / 2}(x)\right) \cap \Gamma\right) \geqq 3 r / 2$ and

$$
1=\lim _{r \rightarrow 0} \frac{\mathcal{H}^{1}\left(B_{r}(x) \cap \Gamma\right)}{2 r} \geqq \lim _{r \rightarrow 0} \frac{\mathcal{H}^{1}\left(B_{r / 2}(x) \cap \Gamma\right)}{2 r}+\frac{3}{4}=\frac{5}{4},
$$

which is a contradiction.

Proposition A.4. (Properties of regular points $[3,29,36]$ ) Suppose that $K \subset \mathbb{R}^{2}$ be a connected compact set with $\mathcal{H}^{1}(K)<\infty$. Thus, it admits a unit (measure-theoretic) normal $v_{K}(x)$ at $\mathcal{H}^{1}$-a.e. $x \in K$; the map $x \mapsto v_{K}(x)$ is Borel measurable and if $L$ is any connected subset of $K$ then $v_{L}(x)=v_{K}(x)$ for any $x \in L$ for which the unit normal $v_{K}(x)$ to $K$ exists. Moreover, $\mathcal{H}^{1}\left\llcorner\sigma_{\rho, x}(K) \rightarrow^{*} \mathcal{H}^{1}\left\llcorner T_{x}\right.\right.$ and $\overline{U_{1, v_{K}}(x)} \cap \sigma_{\rho, x}(K) \stackrel{\mathcal{K}}{\rightarrow} \overline{U_{1, v_{K}}(x)} \cap T_{x}$ as $\rho \rightarrow 0^{+}$, where $T_{x}$ is the generalized tangent line to $K$ at $x$.

Note that $U_{1, v_{K}}(x)$ in Proposition A.4 can be replaced by arbitrary $U_{R, v_{K}}(x)$.

Proposition A.5. Let $A \in \mathcal{A}$ and $x \in \partial A$ be such that the measure-theoretic unit normal $v_{A}(x)$ to $\partial A$ exists and $\overline{U_{R, v_{A}(x)}(x)} \cap \sigma_{\rho, x}(\partial A) \stackrel{\mathcal{K}}{\rightarrow} \overline{U_{R, v_{A}(x)}(x)} \cap T_{x}$ for any $R>0$ as $\rho \rightarrow 0^{+}$. Then:

(a) if $x \in A^{(1)} \cap \partial A$, then $\operatorname{sdist}\left(\cdot, \sigma_{\rho, x}(\partial A)\right) \rightarrow-\operatorname{dist}\left(\cdot, T_{x}\right)$ uniformly in $\overline{U_{1, v_{A}(x)}}$;

(b) if $x \in A^{(0)} \cap \partial A$, then $\operatorname{sdist}\left(\cdot, \sigma_{\rho, x}(\partial A)\right) \rightarrow \operatorname{dist}\left(\cdot, T_{x}\right)$ uniformly in $\overline{U_{1, v_{A}(x)}}$.

Proof. We prove only (a); the proof of (b) is analogous. Let $x \in A^{(1)} \cap \partial A$ be such that

(x1) $v_{A}(x)$ exists;

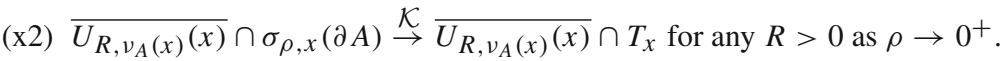

Without loss of generality we assume $x=0, v_{A}(x)=\mathbf{e}_{2}$ and $T_{x}=T_{0}$ is the $x_{1}$-axis. For shortness we write $A_{\rho}$ and $(\partial A)_{\rho}$ in places of $\sigma_{\rho, x}(A)$ and $\sigma_{\rho, x}(\partial A)$, respectively. Let $\left\{\rho_{k}\right\} \subset(0,1)$ be arbitrary sequence converging to 0 . Consider $f_{k}:=\left.\operatorname{sdist}\left(\cdot,(\partial A)_{\rho_{k}}\right)\right|_{U_{4}} \in$ $W^{1, \infty}\left(U_{4}\right)$. For any $k \geqq 1, f_{k}$ is 1 -Lipschitz and $f_{k}(0)=0$, therefore, by the ArzelaAscoli Theorem, there exist $f \in W^{1, \infty}\left(U_{4}\right)$ and not relabelled subsequence $\left\{f_{k}\right\}$ such that $f_{k} \rightarrow f$ uniformly in $U_{4}$. By (x2) applied with $R>4,\left|f_{k}\right|=\operatorname{dist}\left(\cdot,(\partial A)_{\rho_{k}}\right) \rightarrow \operatorname{dist}\left(\cdot, T_{0}\right)$ uniformly in $U_{4}$, therefore, $|f(x)|=\operatorname{dist}\left(x, T_{0}\right)$ for any $x \in U_{4}$. Thus, it suffices to prove that $f \leqq 0$.

Assume by contradiction that $f>0$ in $U_{4}^{+}:=U_{4} \cap\left\{x_{2}>0\right\}$. In addition, by (x2) for any $\delta \in(0,1)$ there exists $k_{\delta}>0$ such that $U_{4} \cap(\partial A)_{\rho_{k}} \subset T_{0} \times(-\delta, \delta)$ for any $k>k_{\delta}$. Therefore, $\operatorname{sdist}\left(\cdot,(\partial A)_{\rho_{k}}\right)>0$ in $U_{4, \delta}^{+}:=U_{4} \cap\left\{x_{2}>\delta\right\}$, and hence, $A \cap \rho_{k} U_{4, \delta}^{+}=\emptyset$, where $r D=\{r x: x \in D\}$. Since $0 \in A^{(1)} \cap \partial A$, this implies

$$
1=\lim _{k \rightarrow \infty} \frac{\left|A \cap U_{4 \rho_{k}}\right|}{\left|U_{4 \rho_{k}}\right|}=\lim _{k \rightarrow \infty} \frac{\left|\left(A \cap U_{4 \rho_{k}}\right) \backslash \rho_{k} U_{4, \delta}^{+}\right|}{\left|U_{4 \rho_{k}}\right|}<1,
$$

which is a contradiction.

An analogous contradiction is obtained assuming $f>0$ in $U_{4} \cap\left\{x_{2}<0\right\}$. 


\section{A.3. Minimizers of Volume-Constraint and Unconstraint Problems}

The following proposition is an extension of [28, Theorem 1.1].

Proposition A.6. Assume (H1)-(H3). Then there exists $\lambda_{0}>0$ (possibly depending on $c_{1}$, $c_{2}$ and $\left.\Omega\right)$ with the following property: given $m \geqq 1,(A, u) \in \mathcal{C}_{m}$ is a solution of $(\mathrm{CP})$ if and only if $(A, u)$ is also a solution to (UP) for all $\lambda \geqq \lambda_{0}$.

Proof. Note that any minimizer $(A, u) \in \mathcal{C}_{m}$ of $\mathcal{F}^{\lambda}$ with $|A|=\mathrm{v}$ is also minimizer of $\mathcal{F}$. Hence, it suffices to show that there exists $\lambda_{0}>0$ such that any minimizer $(A, u)$ of $\mathcal{F}^{\lambda}$ for $\lambda>\lambda_{0}$ satisfies $|A|=\mathrm{v}$.

Assume by contradiction that there exist sequences $\left\{m_{h}\right\} \subset \mathbb{N}$ and $\left\{\lambda_{h}\right\} \subset \mathbb{R}$ with $\lambda_{h} \rightarrow \infty$ and a sequence $\left(A_{h}, u_{h}\right) \in \mathcal{C}_{m_{h}}$ minimizing $\mathcal{F}^{\lambda_{h}}$ such that $\left|A_{h}\right| \neq v$. Since $\Omega$ has finitely many connected components there exists an open Lipschitz set $A_{0} \subset \Omega$ with $\left|A_{0}\right|=\mathrm{v}$ such that $\mathcal{F}^{\lambda_{h}}\left(A_{h}, u_{h}\right) \leqq \mathcal{F}^{\lambda_{h}}\left(A_{0}, u_{0}\right)=\mathcal{F}\left(A_{0}, u_{0}\right)$ for all large $h$. Thus, by (2.4) and (2.5),

$$
\sup _{h} \mathcal{H}^{1}\left(\partial A_{h}\right) \leqq \Lambda:=\frac{\mathcal{F}\left(A_{0}, u_{0}\right)+c_{2} \mathcal{H}^{1}(\Sigma)+\mathcal{H}^{1}(\partial \Omega)}{c_{1}},
$$

and $\lambda_{h}|| A_{h}|-\mathrm{v}| \leqq \mathcal{F}\left(A_{0}, u_{0}\right)+c_{2} \mathcal{H}^{1}(\Sigma)$ for any $h$. This implies $\left|E_{h}\right| \rightarrow \mathrm{v}$ as $h \rightarrow \infty$. By compactness, there exists a finite perimeter set $A \subset \Omega$ and a not relabelled subsequence such that $\chi_{A_{h}} \rightarrow \chi_{A}$ a.e. in $\mathbb{R}^{2}$. In particular, $|A|=\mathrm{v}$.

We suppose that $\left|A_{h}\right|<\mathrm{v}$ for all $h$; the case $\left|A_{h}\right|>\mathrm{v}$ is treated analogously. As in the proof of [28, Theorem 1.1] given $\epsilon \in\left(0, \frac{1}{8}\right)$, there exist small $r>0$ and $x_{r} \in \Omega$ such that $B_{r}(x) \subset \subset \Omega$ and

$$
\left|A \cap B_{r / 2}\left(x_{r}\right)\right|<\epsilon r^{2}, \quad\left|A \cap B_{r}\left(x_{r}\right)\right|>\frac{\pi r^{2}}{16} .
$$

For shortness, we suppose that $x_{r}=0$ we write $B_{r}:=B_{r}\left(x_{r}\right)$. Since $A_{h} \rightarrow A$ in $L^{1}\left(\mathbb{R}^{2}\right)$, for all large $h$,

$$
\left|A_{h} \cap B_{r / 2}\right|<\epsilon r^{2}, \quad\left|A_{h} \cap B_{r}\right|>\frac{\pi r^{2}}{16} .
$$

Let $\Phi: \mathbb{R}^{2} \rightarrow \mathbb{R}^{2}$ be the bi-Lipschitz map which takes $B_{r}$ into $B_{r}$ defined as

$$
\Phi(x):= \begin{cases}(1-3 \sigma) x, & |x|<\frac{r}{2}, \\ x+\sigma\left(1-\frac{r^{2}}{|x|^{2}}\right) x, & \frac{r}{2} \leqq x<r, \\ x, & |x| \geqq r\end{cases}
$$

for some $\sigma \in\left(0, \frac{1}{16}\right)$. Recall from [28, pp. 420-422] that the Jacobian $J \Phi$ of $\Phi$ satisfies

$$
J \Phi(y) \geqq 1+\sigma \quad y \in B_{r} \backslash B_{r / 2},
$$

and

$$
J \Phi(y) \leqq 1+8 \sigma \quad y \in B_{r},
$$

and the tangential Jacobian $J_{1} T_{x}$ of $\Phi$ on the tangent space $T_{x}$ of $\partial A_{h}$ satisfies

$$
J_{1} T_{x} \leqq 1+5 \sigma, \quad x \in B_{r} \cap \partial A_{h} .
$$

Set

$$
E_{h}:=\Phi\left(A_{h}\right) \backslash \partial B_{r}, \quad v_{h}:=u_{h} \chi_{A_{h}} \backslash B_{r}+u_{0} \chi_{E_{h} \cap B_{r}} .
$$


Note that $\left|E_{h}\right|<\mathrm{v}$ and since the bi-Lipschitz maps do not increase the number of connected components, $\left(E_{h}, v_{h}\right) \in \mathcal{C}_{m}$. Let us estimate

$$
\begin{aligned}
\mathcal{F}^{\lambda_{h}} & \left(A_{h}, u_{h}\right)-\mathcal{F}^{\lambda_{h}}\left(E_{h}, v_{h}\right) \\
= & \int_{\overline{B_{r}} \cap A_{h}} \theta_{A_{h}}(x) \phi\left(x, v_{A_{h}}\right) \mathrm{d} \mathcal{H}^{1}-\int_{\overline{B_{r}} \cap \partial E_{h}} \theta_{E_{h}}(x) \phi\left(x, v_{E_{h}}\right) \mathrm{d} \mathcal{H}^{1} \\
& +\int_{B_{r} \cap A_{h}} W\left(x, e\left(u_{h}\right)-E_{0}\right) \mathrm{d} x-\int_{B_{r} \cap E_{h}} W\left(x, e\left(v_{h}\right)-E_{0}\right) \mathrm{d} x \\
& +\lambda_{h}\left(\left|E_{h}\right|-\left|A_{h}\right|\right):=I_{1}+I_{2}+I_{3},
\end{aligned}
$$

where $\theta_{F}(x)$ is 1 for $\mathcal{H}^{1}$-a.e. on $\partial^{*} F$, is 2 for $\mathcal{H}^{1}$-a.e. on $F^{(1)} \cup F^{(0)} \cap \partial F$ and is 0 otherwise. By the choice of $v_{h}, I_{2} \geqq 0$. Moreover, by (A.4) and the area formula as well as from (2.4), (A.2) and equality $\theta_{E_{h}}(\Phi(y))=\theta_{A_{h}}(y)$ for $\mathcal{H}^{1}$-a.e. $y \in \partial A_{h}$,

$$
\begin{gathered}
\int_{B_{r} \cap \partial E_{h}} \theta_{E_{h}}(x) \phi\left(x, v_{E_{h}}\right) \mathrm{d} \mathcal{H}^{1}=\int_{B_{r} \cap \partial A_{h}} \theta_{A_{h}}(\Phi(y)) \phi\left(\Phi(y), v_{A_{h}}\right) J_{1} T_{y} \mathrm{~d} \mathcal{H}^{1} \\
\leqq 2 c_{2}(1+5 \sigma) \mathcal{H}^{1}\left(B_{r} \cap \partial A_{h}\right) \leqq 2 c_{2}(1+5 \sigma) \Lambda
\end{gathered}
$$

Moreover, by (2.4),

$$
\int_{\partial B_{r} \cap \partial E_{h}} \theta_{E_{h}}(x) \phi\left(x, v_{E_{h}}\right) \mathrm{d} \mathcal{H}^{1} \leqq 2 c_{2} \mathcal{H}^{1}\left(\partial B_{r}\right) \leqq 4 \pi c_{2} r,
$$

thus,

$$
I_{1} \geqq-2 c_{2}(1+5 \sigma) \Lambda-4 \pi c_{2} r .
$$

Finally, repeating the same arguments of Step 4 in the proof of [28, Theorem 1.1], we obtain

$$
I_{3} \geqq \lambda_{h} \sigma r^{2}(1-7 \epsilon),
$$

thus,

$$
\mathcal{F}^{\lambda_{h}}\left(A_{h}, u_{h}\right)-\mathcal{F}^{\lambda_{h}}\left(E_{h}, v_{h}\right) \geqq \lambda_{h} \sigma r^{2}(1-7 \epsilon)-2 c_{2}(1+5 \sigma) \Lambda-4 \pi c_{2} r .
$$

Since the dependence of the right-side of (A.5) on $h$ is only through $\lambda_{h}$, for sufficiently large $h$ we have $\mathcal{F}^{\lambda_{h}}\left(A_{h}, u_{h}\right)>\mathcal{F}^{\lambda_{h}}\left(E_{h}, v_{h}\right)$, which contradicts to the minimality of $\left(A_{h}, u_{h}\right)$.

\section{References}

1. Almi, S., Dal Maso, G., Toader, R.: A lower semicontinuity result for a free discontinuity functional with a boundary term. J. Math. Pures Appl. 108, 952-990, 2017

2. Ambrosio, L., Coscia, A., Dal Maso, G.: Fine properties of functions with bounded deformation. Arch. Rational Mech. Anal. 139, 201-238, 1997

3. Ambrosio, L., Fusco, N., Pallara, D.: Functions of Bounded Variation and Free Discontinuity problems. Oxford University Press, New York 2000

4. Asaro, R., Tiller, W.: Interface morphology development during stress corrosion cracking: Part I: via surface diffusion. Metall. Trans. 3, 1789-1796, 1972

5. Babadjian, J.-F., HenaO, D.: Reduced models for linearly elastic thin films allowing for fracture, debonding or delamination. Interface Free Bound. 18, 545-578, 2016 
6. Bella, P., Goldman, M., Zwicknagl, B.: Study of island formation in epitaxially strained films on unbounded domains. Arch. Rational Mech. Anal. 218, 163-217, 2015

7. Bellettini, G., Coscia, A., Dal Maso, G.: Compactness and lower semicontinuity properties in $S B D(\Omega)$. Math. Z. 228, 337-351, 1998

8. Bouchitté, G., Fonseca, I., Mascarenhas, L.: A global method for relaxation. Arch. Rational Mech. Anal. 145, 51-98, 1998

9. Bourdin, B., FrancFort, G.A., Marigo, J.-J.: The variational approach to fracture. J. Elasticity 91, 5-148, 2008

10. Braides, A., Chambolle, A., Solci, M.: A relaxation result for energies defined on pairs set-function and applications. ESAIM Control Optim. Calc. Var. 13, 717-734, 2007

11. Caffarelli, L., Mellet, A.: Capillary drops: contact angle hysteresis and sticking drops. Calc. Var. Partial Differ. Equ. 29, 141-160, 2007

12. Chambolle, A., Conti, S., Francfort, G.: Korn-Poincaré inequalities for functions with a small jump set. Indiana Univ. Math. J. 65, 1373-1399, 2016

13. Chambolle, A., Conti, S., Iurlano, F.: Approximation of functions with small jump sets and existence of strong minimizers of Griffith's energy. J. Math. Pures Appl. 128, 119-139, 2019

14. Chambolle, A., Crismale, V.: A density result in $G S B D^{p}$ with applications to the approximation of brittle fracture energies. Arch. Rational Mech. Anal. 232, 1329-1378, 2019

15. Chambolle, A., Crismale, V.: Compactness and lower-semicontinuity in GSBD. J. Eur. Math. Soc. (JEMS), to appear. arXiv:1802.03302 [math.FA].

16. Conti, S., Focardi, M., Iurlano, F.: Existence of minimizers for the $2 \mathrm{~d}$ stationary Griffith fracture model. C.R. Math. 354 (2016), 1055-1059.

17. Conti, S., Focardi, M., Iurlano, F.: Existence of strong minimizers for the Griffith static fracture model in dimension two. Ann. I. H. Poincaré - AN 36, 455-474, 2019

18. Dal Maso, G.: An Introduction to $\Gamma$-Convergence. Birkhäuser, Boston 1993

19. Dal Maso, G.: Generalised functions of bounded deformation. J. Eur. Math. Soc. 15, 1943-1997, 2013

20. Dal Maso, G., Morel, J., Solimini, S.: A variational method in image segmentation: Existence and approximation results. Acta Math. 168, 89-151, 1992

21. Danescu, A.: The Asaro-Tiller-Grinfeld instability revisited. Int. J. Solids Struct. 38, 4671-4684, 2001

22. Davoli, E., Piovano, P.: Derivation of a heteroepitaxial thin-film model. Interfaces Free Bound. 22-1, 1-26, 2020

23. Davoli, E., Piovano, P.: Analytical validation of the Yound-Dupré law for epitaxiallystrained thin films. Math. Models Methods Appl. Sci. 29, 2183-2223, 2019

24. De Gennes, P.-G., Brochard-Wyart, F., Quéré, D.: Capillarity and Wetting Phenomena: Drops, Bubbles. Pearls. Waves. Springer, New York 2004

25. De Giorgi, E., Buttazzo, G., Dal Maso, G.: On the lower semicontinuity of certain integral functionals. Atti Accad. Naz. Lin. Cl. Sc. Fis. Mat. Nat. Rend. Serie 8 74, 274282, 1983

26. De PhilipPIs, G., Maggi, F.: Regularity of free boundaries in anisotropic capillarity problems and the validity of Young's law. Arch. Rational Mech. Anal. 216, 473-568, 2015

27. Deng, X.: Mechanics of debonding and delamination in composites: asymptotic studies. Compos. Eng. 5, 1299-1315, 1995

28. Esposito, L., Fusco, N.: A remark on a free interface problem with volume constraint. J. Convex Anal. 18, 417-426, 2011

29. FAlConer, K.: The Geometry of Fractal Sets. Cambridge University Press, Cambridge 1985

30. Fonseca, I., Fusco, N., Leoni, G., Millot, V.: Material voids in elastic solids with anisotropic surface energies. J. Math. Pures Appl. 96, 591-639, 2011 
31. Fonseca, I., Fusco, N., Leoni, G., Morini, M.: Equilibrium configurations of epitaxially strained crystalline films: existence and regularity results. Arch. Rational Mech. Anal. 186, 477-537, 2007

32. Fonseca, I., Leoni, G.: Modern Methods in the Calculus of Variations: $L^{p}$ Spaces. Springer, New York 2007

33. Fried, E., Gurtin, M.: A unified treatment of evolving interfaces accounting for small deformations and atomic transport with emphasis on grain-boundaries and epitaxy. Adv. Appl. Mech. 40, 1-177, 2004

34. Fusco, N., Julin, V., Morini, M.: The surface diffusion flow with elasticity in the plane. Commun. Math. Phys. 362, 571-607, 2018

35. Fusco, N., Morini, M.: Equilibrium configurations of epitaxially strained elastic films: second order minimality conditions and qualitative properties of solutions. Arch. Rational Mech. Anal. 203, 247-327, 2012

36. Giacomini, A.: A generalization of Golab's theorem and applications to fracture mechanics. Math. Models Methods Appl. Sci. 12, 1245-1267, 2002

37. Giusti, E.: Minimal Surfaces and Functions of Bounded Variation. Birkhäuser, Boston 1984

38. Goldman, M., Zwicknagl, B.: Scaling law and reduced models for epitaxially strained crystalline films. SIAM J. Math. Anal. 46, 1-24, 2014

39. Griffith, A.A.: The phenomena of rupture and flow in solids. Philos. Trans. R. Soc. Lond. A 221, 163-198, 1921

40. GRINFELD, M.A.: The stress driven instabilities in crystals: mathematical models and physical manifestations. J. Nonlinear Sci. 3, 35-83, 1993

41. Hutchinson, J., Suo, Z.: Mixed mode cracking in layered materials. Adv. Appl. Mech. 29, 63-191, 1991

42. Kreutz L., Piovano P.: Microscopic validation of a variational model of epitaxially strained crystalline films. Submitted (2019), arXiv:1902.06561 [math.AP].

43. León Baldelli, A.A., Babadjian, J.-F., Bourdin, B., Henao, D., Maurini, C.: A variational model for fracture and debonding of thin films under in-plane loadings. $J$. Mech. Phys. Solids 70, 320-348, 2014

44. León Baldelli, A.A., Bourdin, B., Marigo, J.-J., Maurini, C.: Fracture and debonding of a thin film on a stiff substrate: analytical and numerical solutions of a onedimensional variational model. Cont. Mech. Thermodyn. 25, 243-268, 2013

45. Maddalena, F., Solimini, S.: Lower semicontinuity properties of functionals with free discontinuities. Arch. Rational Mech. Anal. 159, 273-294, 2001

46. MagGi, F.: Sets of Finite Perimeter and Geometric Variational Problems: An Introduction to Geometric Measure Theory. Cambridge University Press, Cambridge 2012

47. Siegel, M., Miksis, M., Voorhees, P.: Evolution of material voids for highly anisotropic surface energy. J. Mech. Phys. Solids 52, 1319-1353, 2004

48. SPENCER, B.J.: Asymptotic derivation of the glued-wetting-layer model and the contactangle condition for Stranski-Krastanow islands. Phys. Rev. B 59, 2011-2017, 1999

49. WANG, H., Li, Z.: The instability of the diffusion-controlled grain-boundary void in stressed solid. Acta Mech. Sinica 19, 330-339, 2003

50. Xia, Z.C., Hutchinson, J.W.: Crack patterns in thin films. J. Mech. Phys. Solids 48, 1107-1131, 2000 
S. Y. Kholmatov\& P. Piovano Fakultät für Mathematik, Universität Wien,

Oskar-Morgenstern Platz 1, 1090 Wien

Austria.

ShoKhrukh Yu. Kholmatov

e-mail: shokhrukh.kholmatov@univie.ac.at

Paolo Piovano

e-mail: paolo.piovano@univie.ac.at

(Received February 18, 2019 / Accepted May 21, 2020)

Published online June 20, 2020

(c) The Author(s) (2020) 